\title{
Catalogue of type specimens of beetles (Coleoptera) deposited in the National Museum, Prague, Czech Republic ${ }^{1)}$
}

\section{Staphylinidae: Staphylininae: Staphylinini: Philonthina}

\author{
Šárka MIKÁTOVÁ, Lenka MACHÁČKOVÁ \& Jiří HÁJEK*) \\ Department of Entomology, National Museum, Cirkusová 1740, CZ-193 00 Praha 9 - Horní Počernice, Czech Republic \\ *) corresponding author: e-mail: jiri_hajek@nm.cz
}

Accepted: $24^{\text {th }}$ March 2020

Published online: $15^{\text {th }}$ May 2020

\begin{abstract}
Type specimens from the collection of beetles (Coleoptera) deposited in the Department of Entomology, National Museum, Prague, are currently being catalogued. In this part of the catalogue dealing with the Staphylinini subtribe Philonthina (Staphylinidae: Staphylininae) we present information on types of 278 species level taxa. Except for the general collection, the type material comes mostly from the recently acquired collection of the late Lubomír Hromádka. A new substitute name, Philonthus acervulus nom. nov., is proposed for Philonthus zosterops Hromádka, 2013, a junior primary homonym of Philonthus zosterops Hromádka, 2011. One new combination is established: Eccoptolonthus terezae (Hromádka, 2016) comb. nov. from Pseudohesperus Hayashi, 2008. Philonthus zosterops Hromádka, 2011 is synonymized with P. mesophoyx Hromádka, 2016, syn. nov. Correct original spellings of Philonthus terathopius Hromádka, 2012 and Philonthus crecopsis Hromádka, 2013 are fixed. The type locality and distribution of Philonthus camelus Hromádka, 2008 is corrected from Tanzania: Kilimandjaro to the Republic of the Congo: Bouansa.
\end{abstract}

Key words. Coleoptera, Staphylinidae, Philonthina, catalogue, types, National Museum Prague, new substitute name, new synonym, new combination, correct original spelling

Zoobank: http://zoobank.org/urn:lsid:zoobank.org:pub:5FFDC0A8-BCC8-45B4-B9A5-7C372E129707

(C) 2020 The Authors. This work is licensed under the Creative Commons Attribution-NonCommercial-NoDerivs 3.0 Licence.

\section{Introduction}

The Department of Entomology of the National Museum, Prague (NMP; NMPC when referring to the collection) holds an important collection of insects from all zoogeographical regions, including numerous species-group type specimens, whose number is estimated to be several tens of thousands (the majority of them belonging to Coleoptera), and the presence of some of them in the collection is still largely unknown. Following the International Code of Zoological Nomenclature which encourages institutions to catalogue and make accessible the type material under their care (ICZN 1999: Recommendation 72F), BEZDĚK \& HÁJEK (2009) started with cataloguing types in the NMPC to improve the knowledge about the collections and provide information to the entomological community. This effort has already resulted in 16 parts, most of them devoted to the beetle

${ }^{1)}$ Catalogue of type specimens in NMPC, Part 17 superfamily Scarabaeoidea (see BEZDĚK et al. 2017), other Coleoptera families (e.g. BATELKA \& HÁJEK 2015), as well as Polyneoptera (MACHÁČKOvÁ \& FIKÁČEK 2014), Hemiptera: Heteroptera (e.g. KMENT et al. 2015) and Sternorrhyncha (MalenovskÝ et al. 2016), Diptera (TKoČ et al. 2014), and Hymenoptera (BEZDĚČKová et al. 2017). One part of the catalogue was devoted to the Staphylinidae subfamilies Euaesthetinae, Leptotyphlinae, Megalopsidiinae, Oxyporinae and Steninae (MACHÁČKOVÁ et al. 2017). In the present part, we continue cataloguing the family Staphylinidae, namely the subtribe Philonthina of the tribe Staphylinini. Along with the paper, photos of most types and copies of their original descriptions are available on request.

As in the previous parts, we present brief information about the most important collections/specialists mentioned in the catalogue:

The collection of Lubomír Hromádka (1931-2016) represents one of the largest specialised collections of 
the Staphylinidae in the NMP. Hromádka, a men's tailor and enthusiastic amateur entomologist, focused on Staphylinidae from the 1960's; first he was interested mainly in Steninae and after his retirement he switched to the Afrotropical Staphylininae: Philonthina. He did not collect beetles much himself but he processed material for various institutions (including the NMPC) as well as for private collectors, and thus his collection (comprising over 30,000 specimens) was built mainly from donations, exchanges and desiderata. Most species in this catalogue were described by L. Hromádka himself, several additional taxa were described by J. Janák and A. Smetana (see also below). For a biography and bibliography of L. Hromádka see HERMAN (2001a: 81) and JANÁK $(2011,2017)$.

The general collection of the Staphylinidae in the NMP was established by uniting the collections of several important staphylinid specialists. For this part of the catalogue, the most important is František Rambousek (1886-1931) - a zoologist working at the Institute of Sugar Industry, and a specialist on the Staphylinidae taxonomy; his collection contains about 70,000 specimens from all parts of the world, including dozens of type specimens of taxa described by Rambousek himself. Other important collections are those of Josef Fleischer (1866-1940), Štěpán Jureček (1877-1940), or Václav Machulka (1889-1949). These collections were supplemented with rich material from the museum's entomological expeditions to Turkey (1947) and Iran (1970, 1973, and 1977). Most recently, scientific treatment of the material newly collected by the museum employees (especially to China, Laos or Yemen) brought more Staphylinidae types to the NMPC. Max Bernhauer (1866-1946), born in Moravia, participated most in the scientific treatment of the old collection. In 1950's, Aleš Smetana (born 1931), during 1970-1971 shortly employed at the NMP, started his work on staphylinids and processed a large part of the then collection. From 1970, L. Hromádka studied and described new taxa from the NMPC. Most recently, Harald Schillhammer (born 1960) contributed to the scientific treatment of material from the Oriental Region.

\section{Material and methods}

We tentatively follow Herman's (2001b) catalogue and the phylogeny of CHANI-Posse DE MAUs et al. (2018) for generic classification within the subtribe Phinonthina. The genera and species are arranged alphabetically. Each entry includes:

- the name of the taxon in its original combination.

- the name of the taxon in its original combination and spelling, with the author and year of description. Pagination, figures and plates are also given.

- the name-bearing type, number of specimens (including sex if known) and exact label data. Our remarks are found in square brackets: [p] - the preceding data are printed, [hw] - the preceding data are handwritten. Separate labels are indicated by a double slash '//' and lines within each label are separated by a slash ' $/$.

- the current taxonomic status. The genus Philonthus Stephens, 1829 is currently considered polyphyletic and the status of its subgenera is not clear (CHANI-Posse DE MaUs et al. 2018). Therefore, the formally valid subgenera of Philonthus are largely omitted by recent authors, with use of informal species groups instead of the subgenera (see HromÁDKA 2008a,b,c, 2009c,d, 2010a,c,d,e, 2011b,c,e, 2012c,d,e,f, 2013c,d,e). Following that, we do not mention Philonthus subgenera in this catalogue.

- any taxonomic problems and inconsistencies are mentioned under Comments.

A full reference to each publication can be found in References.

\section{Catalogue}

Staphylinidae

Staphylininae

Staphylinini: Philonthina

\section{Afrorabigus scopus Hromádka, 2014}

Afrorabigus scopus Hromádka, 2014a: 684, Figs 1-3.

The holotype and one paratype are deposited in NMPC (general collection and ex coll. L. Hromádka):

HoLotype (đ̊): ‘Afrika Tanga [sic!] / VIII 1902 / Methner [hw] // ex coll. L. Hromádka / National Museum / Prague, Czech Republic [p] // HOLOTYPUS / AFRORABIGUS / scopus sp. nov. / Hromádka, det., 2013. [p, red label]'.

Paratype (P): ‘Afrika Tanga [sic!] / VIII.1902 / Methner [hw] // ex coll. L. Hromádka / National Museum / Prague, Czech Republic [p] // PARATY PUS / AFRORABIGUS / scopus sp. nov. / Hromádka, det., 2013. [p, red label]'.

Current status. Valid species.

Afrorabigus tropheus Hromádka, 2011

Afrorabigus tropheus Hromádka, 2011d: 74, Figs 21-23.

Two paratypes are deposited in NMPC (ex coll. L. Hromádka):

ParatyPe (d): ‘ETHIOPIA: Bale / 8km. W. of Dinshu / 0706 N. 3944 E. / 3.050m. xii. 1971 [p] // R.O.S.,Clark / B.M. 973-450 [p] // Under damp / vegetation [p] // ex coll. L. Hromádka / National Museum / Prague, Czech Republic [p] // PARATYPUS / AFRORABIGUS Levasseur / tropheus sp. nov. / Hromádka, det., 2010 [p, red label]'.

ParatyPe (đ): 'ETHIOPIA:Bale / Sof Omar 1.200 m. / 0704N.4036E. / xii.1971 [p] // R.O.S.,Clark / B.M. 973-450 [p] // Under damp / vegetation [p] // ex coll. L. Hromádka / National Museum / Prague, Czech Republic [p] // PARATY PUS / AFRORABIGUS Levasseur / tropheus sp. nov. / Hromádka, det., 2010 [p, red label]’.

Current status. Valid species.

\section{Belonuchus oceanites Hromádka, 2016}

Belonochus [sic!] oceanites Hromádka, 2016a: 102, Figs 4-7.

The holotype is deposited in NMPC (ex coll. L. Hromád$\mathrm{ka})$ :

HoLotype (ð’): ‘NAMIBIA,Kuzikus W.R. / 2314'17.2”'S 18²3'28.8”'E [sic!] / Pitfall / 29-III $\rightarrow$ 03-IV-2011 / I.G.31.840 / Leg J.Constant [sic!] [p, blue label] // ex coll. L. Hromádka / National Museum / Prague, Czech Republic [p] // HOLOTYPUS / BELONOCHUS / oceanites sp. nov. / Hromádka, det., 2015. [p, red label]'.

Current status. 'Belonuchus' oceanites Hromádka, 2016. Comments. Chani-Posse de Maus et al. (2018) redefined the concept of the genus Belonuchus Nordmann, 1837 as strictly New World lineage, and transferred 38 Old World 
Belonuchus to the reinstated genus Trapeziderus Motschulsky, 1860. However, another 20 species, including B. oceanites, were not mentioned, most probably because the authors thought those species do not belong to Trapeziderus. Therefore, we retain the species as 'Belonuchus' oceanites until its taxonomic revision.

\section{Belonuchus pectinipes Bernhauer, 1910}

Belonuchus pectinipes Bernhauer, 1910: 379.

One syntype is deposited in NMPC (general collection): SYNTYPE (unsexed specimen): ‘ $+[\mathrm{hw}] / /$ Cordoba / Mex Ver. / Dr.A.Fenyes [p] // pectinipes / Brh. Cotypus [hw]'.

Current status. Valid species, see Herman (2001b).

\section{Bisnius agenor Smetana, 1995}

Bisnius agenor Smetana, 1995: 591, Figs 954-959.

Two paratypes are deposited in NMPC (ex coll. L. Hromádka):

Paratypes (1 $\partial, 1$ unsexed specimen): 'WASH.Mt.Rainier N.P. / Tahoma Crk.2400، / 10.VIII.1973 / A \& Z \& D Smetana [p] // ex coll. L. Hromádka / National Museum / Prague, Czech Republic [p] // PARATYPE / Bisnius / agenor / A. Smetana 1993 / CNC No.21988 [p, yellow label]'

Current status. Valid species, see Herman (2001b).

\section{Bisnius picicornis zetes Smetana, 1995}

Bisnius picicornis zetes Smetana, 1995: 562, Figs 873-880.

Two paratypes are deposited in NMPC (ex coll. L. Hromádka):

PARATyPe (unsexed specimen): 'N.M.Lincoln N.F.2mi. / E.Cloudcroft 8750` / 15.VII.69 A.Smetana // ex coll. L. Hromádka / National Museum / Prague, Czech Republic [p] // PARATYPE / Bisnius / picicornis zetes / A. Smetana 1993 / CNC No.21979 [p, yellow label]'.

Paratype (unsexed specimen): 'N.M.Lincoln N.F.lmi. / SE Cloudcroft 8750، / 14.VII.69 A.Smetana // ex coll. L. Hromádka / National Museum / Prague, Czech Republic [p] // PARATYPE / Bisnius / picicornis zetes / A. Smetana 1993 / CNC No.21979 [p, yellow label]'.

Current status. Valid subspecies, see Herman (2001b).

\section{Bisnius schillhammeri Hromádka, 2001}

Bisnius schillhammeri Hromádka, 2001: 139, Figs 6-8.

The holotype is deposited in NMPC (general collection):

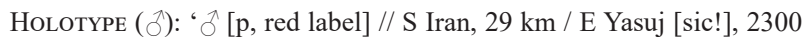
m/16-17.6 1973 [p] // Loc no. 245 / Exp. Nat. Mus / Praha [p] // HOLOTYPUS / Bisnius / schillhammeri sp.n. / L.Hromádka det. 99. [p, red label]'.

Current status. Valid species, see SchülKe \& Smetana (2015) and SCHILLHAMMER (2019).

\section{Gabrius abas Smetana, 1984}

Gabrius abas Smetana, 1984: 135, Figs 22-24.

One paratype is deposited in NMPC (ex coll. L. Hromád$\mathrm{ka})$ :

Paratype (unsexed specimen): 'JAPAN Gumma Pr. / 5km E. Usui Pass / 900m 25.VII.80 / A.\&Z. Smetana [p] // ex coll. L. Hromádka/ National Museum / Prague, Czech Republic [p] // PARAT Y PE / Gabrius abas / A.Smetana [p, yellow label]',
Current status. Valid species, see SCHÜlKe \& SMETANA (2015).

Gabrius accipiter Hromádka, 2014

Gabrius accipiter Hromádka, 2014f: 26, Figs 1-3.

The holotype is deposited in NMPC (general collection): HoLOTYPE ( ${ }^{1}$ ): 'BOTSWANA (B 11) / Moremi Reservs / $19^{\circ} 23^{\circ} \mathrm{S} 23$ ${ }^{\circ} 33 \mathrm{E}$ [sic!] / 18.-20. 1972 [sic!] [p] // ex coll. L. Hromádka / National Museum / Prague, Czech Republic [p] // HOLOTY PUS / GABRIUS Stephens / accipiter sp. nov / Hromádka, det., 2014. [p, red label]'.

Current status. Valid species.

Gabrius amadina Hromádka, 2014

Gabrius amadina Hromádka, 2014c: 560, Figs 34-36.

The holotype is deposited in NMPC (general collection):

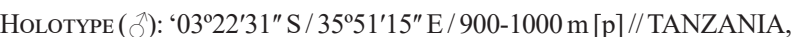
Arusha / distr.,May 01,2010 / MTO-WA-MBU env. / Milan Kubon lgt. [p] // ex coll. L. Hromádka / National Museum / Prague, Czech Republic [p] // HOLOTYPUS / GABRIUS Stephens / amadina sp. nov. / Hromádka, det. 2013. [p, red label]'.

Current status. Valid species.

\section{Gabrius anatolicus Smetana, 1953}

Gabrius anatolicus Smetana, 1953b: 119, Figs 2-3.

The holotype and five paratypes (including the allotype) are deposited in NMPC (general collection):

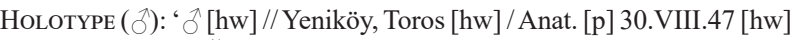
/ Exp. N.Mus, ČSR. [p] // Gabrius Steph. [p] / anatolicus / m. [hw] / det. Smetana 19 [p] 52 [hw] // HOLOTYPE [p, red label] // Mus. Nat. Pragae / Inv. [p] 18768 [hw, orange label]'.

Paratype (q): 'Yeniköy, Toros [hw] / Anat. [p] 30.VIII.47 [hw] / Exp. N.Mus. ČSR. [p] // ALLOTYPE [p, red label] // Gabrius Steph. [p]/ anatolicus / m. [hw]/det. Smetana 19 [p] 52 [hw] // Mus. Nat. Pragae [p] / 18769 [hw] / Inv. [p, orange label]'.

Paratype (ð): 'Yeniköy, Toros [hw] / Anat. [p] 30.VIII.47 [hw] / Exp. N.Mus. ČSR. [p] // coll. Hromádka // PARATYPE [p, red label] // Gabrius Steph. [p]/anatolicus / m. [hw]/det. Smetana 19 [p] 52 [hw]'.

Paratype ( $($ ): 'Yeniköy, Toros [hw] / Anat. [p] 30.VIII.47 [hw] / Exp. N.Mus. ČSR. [p] // PARATYPE [p, red label] // Gabrius Steph. [p]/ anatolicus / m. [hw] / det. Smetana 19 [p] 52 [hw] // Mus. Nat. Pragae [p] / $18770[\mathrm{hw}] /$ Inv. [p, orange label]'.

Paratype ( + ): 'Yeniköy, Toros [hw] / Anat. [p] 30.VIII.47 [hw] / Exp. N.Mus. ČSR. [p] // PARATYPE [p, red label] // Gabrius Steph. [p]/ anatolicus / m. [hw] / det. Smetana 19 [p] 52 [hw] // Mus. Nat. Pragae [p] / 18773 [hw] / Inv. [p, orange label]'.

Paratype (+): 'Yeniköy, Toros [hw] / Anat. [p] 30.VIII.47 [hw] / Exp. N.Mus. ČSR. [p] // PARATYPE [p, red label] // Gabrius Steph. [p]/ anatolicus / m. [hw] / det. Smetana 19 [p] 52 [hw]// Mus. Nat. Pragae [p] / 18772 [hw] / Inv. [p, orange label]'.

Current status. Valid species, see SchülKe \& SMETANA (2015).

\section{Gabrius arctocebus Hromádka, 2014}

Gabrius arctocebus Hromádka, 2014g: 36, Figs 4-6.

The holotype is deposited in NMPC (general collection): Holotype (ð): ‘BOTSWANA(B 11)/ Moremi Reservs / 19 ²3'S $23^{\circ} 33 \mathrm{E}$ [sic!] / 18. - 20. 1972 [sic!] [p] // ex coll. L. Hromádka / National Museum / Prague, Czech Republic [p] // HOLOTYPUS / GABRIUS Step. / arctocebus sp. nov. / Hromádka, det., 2013. [p, red label]'.

Current status. Valid species. 
Gabrius ardea Hromádka, 2014

Gabrius ardea Hromádka, 2014g: 35, Figs 1-3.

The holotype and one paratype are deposited in NMPC (general collection and ex coll. L. Hromádka):

HolotyPe (đ): 'Rep. South Africa / Northern Prov. Camp / David, 5km S. Ofoolaco / 475m 17.-24.i.2002 / leg. S. Murzin [p, green label] // ex coll. L. Hromádka / National Museum / Prague, Czech Republic [p] // HOLOTY PU S GABRIUS Step. / ardea sp. nov. / Hromádka, det. 2013. [p, orange label]'.

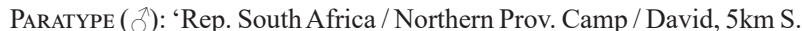
Ofoolaco / 475m 17.-24.i.2002 / leg. S. Murzin [p, green label] // ex coll. L. Hromádka / National Museum / Prague, Czech Republic [p] // PARATYPUS / GABRIUS Stephens / ardea sp. nov. / Hromádka, det., 2013. [p, red label]'.

Current status. Valid species.

\section{Gabrius ardeola Hromádka, 2011}

Gabrius ardeola Hromádka, 2011f: 1378, Figs 1-4.

Two paratypes are deposited in NMPC (ex coll. L. Hromádka):

PARATyPe (ठ): 'E Madagascar, 15.4.2001 / N Andringitra:Vohidray rdg. / $\Delta$ 1920m env., N part / J. Janák lgt.,1900-1920m [p] // rock / under Philippia / sifting [p] // ex coll. L. Hromádka / National Museum / Prague, Czech Republic [p] // PARATYPUS / GABRIUS Stephens / ardeola sp. nov. / Hromádka, det. 2009 [p, orange label]'.

PARATYPe (ठ): 'E Madagascar, 10.-11.4.2001 / N Andringitra:Vohidray rdg. / 3-4km SSE of Amboarafibe / J. Janák lgt., 1600-1700m [p] // rain forest / forest litter / sifting [sic!] [p] // ex coll. L. Hromádka / National Museum / Prague, Czech Republic [p] // PARATY PUS / GABRIUS Stephens / ardeola sp. nov. / Hromádka, det. 2009 [p, orange label]'.

Current status. Valid species.

Gabrius arenaria Hromádka, 2016

Gabrius arenaria Hromádka, 2016d: 93, Figs 1-3.

The holotype is deposited in NMPC (ex coll. L. Hromád$\mathrm{ka)}$ :

Holotype (ð): 'ETHIOPIA: Bale / 8km. W. of Dinshu / 0706 N. 3944 E. / 3.050m. xii. 1971 [p] // ex coll. L. Hromádka / National Museum / Prague, Czech Republic [p] // HOLOTYPUS / GABRIUS Stephens / arenaria sp. nov. / Hromádka det., 2015 [p, orange label]'.

Current status. Valid species.

\section{Gabrius assingi Hromádka, 2016}

Gabrius assingi Hromádka, 2016d: 96, Figs 7-9.

One paratype is deposited in NMPC (ex coll. L. Hromád$\mathrm{ka)}$ :

PARAType ( + ): 'SIERRA LEONE - / Western Area, / Banga Farm near / Sussex, 25.I.2013, / leg. W. Rossi [p] // Philonthus / sp. + / det. V. Assing 2014 [p] // ex coll. L. Hromádka / National Museum / Prague, Czech Republic [p] // PARAT Y PUS / GABRIUS Stephens / assingi sp. nov. / Hromádka, det., 2015. [p, orange label]'.

Current status. Valid species.

\section{Gabrius balaeniceps Hromádka, 2014}

Gabrius balaeniceps Hromádka, 2014g: 38, Figs 7-9.

The holotype and one paratype are deposited in NMPC (general collection and ex coll. L. Hromádka):

Holotype (ð): 'République Centraafricante [sic!] / Bozo lumiere /

21.v.1981. / leg. N. Degallier [p] // ex coll. L. Hromádka / National
Museum / Prague, Czech Republic [p] // HOLOTY PUS / GABRIUS Step. / balaeniceps sp. nov. / Hromádka, det. 2013 [p, orange label]'. Paratype (§): 'République Centraafricante [sic!] / Bozo lumiere / 21.v.1981. / leg. N. Degallier [p] // ex coll. L. Hromádka / National Museum / Prague, Czech Republic [p] // PARATY PUS / GABRIUS Step. / balaeniceps sp. nov. / Hromádka det., 2013. [p, red label]'.

Current status. Valid species.

\section{Gabrius bescidicus Smetana, 1954}

Gabrius bescidicus Smetana, 1954a: 116, Figs 9, 12.

The holotype and five paratypes (including the allotype) are deposited in NMPC (general collection):

HoLotype ( $\left.)^{\prime}\right)$ : ‘ [p] // Bílá / Morava / Machulka [p] // HOLOTYPE [p, red label] // bescidicus / M. [hw] // Gabrius Steph. [p] / bescidicus / m. [hw] / det. Smetana 19 [p] 51 [hw] // Mus. Nat. Pragae / Inv. [p] 18774 [hw, orange label]'.

Paratype ( (): 'Morava / Bílá / Machulka [hw] // ALLOTYPE [p, red label] // Gabrius Steph. [p] / bescidicus / m. [hw] / det. Smetana 19 [p] $51[\mathrm{hw}] / /$ Mus. Nat. Pragae [p] / 18775 [hw] / Inv. [p, orange label]'. PARATYPE (ô): 'Bílá / Morava / Machulka [p] // PARATYPE [p, red label] // Gabrius Steph. [p] / bescidicus / m. [hw]/det. Smetana 19 [p] 51 [hw]'.

PARATyPe (unsexed specimen): 'Morava / Bílá / Machulka [hw] // PARATYPE [p, red label] // Gabrius Steph. [p] / bescidicus / m. [hw] / det. Smetana $19[\mathrm{p}] 51[\mathrm{hw}]$ '.

ParaTYPe (+): 'Morava / Bílá / Machulka [hw] // PARATYPE [p, red label] // Gabrius Steph. [p] / bescidicus / m. [hw] / det. Smetana 19 [p] 51 [hw] // Mus. Nat. Pragae [p] / 18777 [hw] / Inv. [p, orange label]'. PARATYPe (+): 'Morava / Bílá / Machulka [hw] // PARATYPE [p, red label] // Gabrius Steph. [p] / bescidicus / m. [hw] / det. Smetana 19 [p] 51 [hw] // Mus. Nat. Pragae [p] / 18778 [hw] / Inv. [p, orange label]'.

Current status. Valid species, see SchÜLKE \& SMETANA (2015).

Gabrius bugeranus Hromádka, 2015

Gabrius bugeranus Hromádka, 2015c: 28, Figs 4-6.

Three paratypes are deposited in NMPC (ex coll. L. Hromádka):

PARATYPes ( 1 j, 2 unsexed specimens): 'Ethiopia, SNNPR, Bonga area, / Adiyo [sic!] / Gimbo, Boka to Bonga [sic!], car / net: 1777-2668 $\mathrm{m}, 07^{\circ} 17.711^{\prime} \mathrm{N} / 36^{\circ} 22.555^{\prime} \mathrm{E}, 07^{\circ} 15.064^{\prime} \mathrm{N} / 36^{\circ} 15.298^{\prime} \mathrm{E}$ [sic!] 7.XII.2014, Schöller [p] // ex coll. L. Hromádka / National Museum / Prague, Czech Republic [p] // PARATYPUS / GABRIUS Step. / bugeranus sp. nov. / Hromádka, det., 2015 [p, red label]'.

Current status. Valid species.

\section{Gabrius calonectris Hromádka, 2014}

Gabrius calonectris Hromádka, 2014f: 27, Figs 4-8.

The holotype is deposited in NMPC (ex coll. L. Hromád$\mathrm{ka)}$ :

Holotype (ð): 'Tanzania / Uru north, env. $1750 \mathrm{~m} / 16 \mathrm{~km} \mathrm{~N}$ of Moshi.v.2010 / Milan Kuboň lgt. [p] // ex coll. L. Hromádka / National Museum / Prague, Czech Republic [p] // HOLOTYPUS / GABRIUS Stephens / calonectris sp. nov. / Hromádka, det., 2014. [p, orange label]'.

Current status. Valid species.

Gabrius chrysochloris Hromádka, 2014

Gabrius chrysochloris Hromádka, 2014e: 695, Figs 1-3.

The holotype is deposited in NMPC (general collection): HoLOtYPe (ð): 'ETHIOPIA: Bale / 8km. W. of Dinshu / 0706 N. 3944 E. / 3.050m. xii. 1971 [p] // ex coll. L. Hromádka / National Museum / 
Prague, Czech Republic [p] // HOLOTY PUS / GABRIUS Stephens / chrysochloris sp. nov. / Hromádka, det., 2014. [p, red label]'.

Current status. Valid species.

Gabrius chrysococcyx Hromádka, 2014

Gabrius chrysococcyx Hromádka, 2014g: 40, Figs 12-14.

The holotype is deposited in NMPC (general collection): Holotype ( ${ }^{\jmath}$ ): 'SIERRA LEONE / Eastern Prov .Kono / Sandia / 19.v.1991 W. Rossi [p, yellow label] // ex coll. L. Hromádka / National Museum / Prague, Czech Republic [p] // HOLOTYPUS / GABRIUS Step. / chrysococcyx sp. nov. / Hromadka, det. 2013 [p, orange label]'.

Current status. Valid species.

Gabrius coracias Hromádka, 2014

Gabrius coracias Hromádka, 2014h: 174, Figs 7-9.

The holotype is deposited in NMPC (general collection): HoLOtyPe (ð): 'SIERRA LEONE / Western Area / Base Picket Hill / 9.i. 1997 W. Rossi [p, green label] // ex coll. L. Hromádka / National Museum / Prague, Czech Republic [p] // HOLOTYPUS / GABRIUS Stephens / coracias sp. nov. / Hromádka, det., 2012 [p, red label]'.

Current status. Valid species.

\section{Gabrius corythaeola Hromádka, 2014}

Gabrius corythaeola Hromádka, 2014g: 42, Figs 15-17.

Six paratypes are deposited in NMPC (ex coll. L. Hromád$\mathrm{ka)}$ :

Paratypes (3ð̋): 'Musule W.: 2400 m / 24.vii.1964 [sic!] / Tamisage / sous Bambous [p, blue label] // Coll. Mus. Tervuren / Zafre: P. N. Virunga / Volcan Sabinyo / R. P. Cellis, vii.1964 [p, blue label] // ex coll. L. Hromádka / National Museum / Prague, Czech Republic [p] // PARATYPUS / GABRIUS Stephens / corythaeola sp. nov. / Hromádka, det., 2013. [p, red label]'.

PARATYPE (ठ): 'Massif Ruwenzori / Mont Ngulingo / prés Nyamgaleke / 2500 m, ex. P.N.A. [p] // Congo Belge / 29.iv.1954 / P. Vanschuytbroeck \& / H. Synave 8764 [p] // ex coll. L. Hromádka / National Museum / Prague, Czech Republic [p] // PARATYPUS / GABRIUS Step. / corythaeola, sp. nov. / Hromádka det. 2013 [p, orange label]'

Paratype (đ): 'Massif Ruwenzori / Kyandolire 1.750 m / Riv. Mulaku / af. Kakalari (terreau) [p, yellow label] // Congo Belge / 29.iv.1954 / P. Vanschuytbroeck \& / at Kakalari (terreau) [p, yellow label] // ex coll. L. Hromádka / National Museum / Prague, Czech Republic [p] // PARATYPUS / GABRIUS Step. / corythaeola sp. nov. / Hromádka, det., 2013. [p, orange label]'.

Paratype (ð): 'Massif Ruwenzori / Katonge 2010 / Riv. Nyamwamba / Affl. Butahu [p] // Congo Belge: P.N.A. / 2.-3.ii.1953 / P. Vanschuythbroeck / \& J.Kakenbosch [p] // ex coll. L. Hromádka / National Museum / Prague, Czech Republic [p] // PARATYPUS / GABRIUS Step. / corythaeola, sp. nov. / Hromádka det. 2013 [p, orange label]'.

Current status. Valid species.

\section{Gabrius coturnix Hromádka, 2014}

Gabrius coturnix Hromádka, 2014f: 28, Figs 9-13.

The holotype is deposited in NMPC (general collection):

Holotype (ठ)): ‘TANZANIA/Mwanza / 11.x.1969 / Ardö leg. [p] // Under damp / vegetation [p] // ex coll. L. Hromádka / National Museum / Prague, Czech Republic [p] // HOLOT Y PUS / GABRIUS Stephens / coturnix sp. nov. / Hromádka, det., 2014. [p, orange label]'.

Current status. Valid species.
Gabrius cyanolanius Hromádka, 2011

Gabrius cyanolanius Hromádka, 2011f: 1379, Figs 5-8.

One paratype is deposited in NMPC (ex coll. L. Hromádka):

PaRATYPe (đ): 'E Madagascar, 16.12.1998 / 30km SE of Betroka,3km E / of $\boldsymbol{\Lambda} 1656 \mathrm{~m}$ Ambolando [sic!] / 1200m,J. Janák lgt. [sic!] [p] // savanna, / zebu / droppings [p] // COLLECTION / L. Hromádka / PRAHA [p, orange label] // PARATYPUS / GABRIUS Stephens / cyanolanius sp. nov / Hromádka, det. 2009 [p, orange label]'

Current status. Valid species.

Gabrius elephantulus Hromádka, 2014

Gabrius elephantulus Hromádka, 2014e: 697, Figs 4-6.

The holotype is deposited in NMPC (general collection):

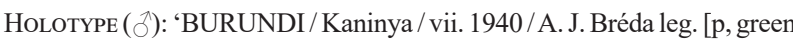
label] // ex coll. L. Hromádka / National Museum / Prague, Czech Republic [p] // HOLOTYPUS / GABRIUS Stephens / elephantulus sp. nov. / Hromádka, det., 2014. [p, red label]'.

Current status. Valid species.

Gabrius epichoristodes Hromádka, 2014

Gabrius epichoristodes Hromádka, 2014g: 43, Figs 18-20.

One paratype is deposited in NMPC (ex coll. L. Hromádka): PARATYPE ( $(ð)$ ): 'COLL. MUS. CONGO / Kivu : contr. S. Kahuzi, / 2200m, 27/III-53 / P. Basilewsky [p] // ex coll. L. Hromádka / National Museum / Prague, Czech Republic [p] // PARATYPUS / GABRIUS Steph. / epichoristodes sp. nov. / Hromádka, det., 2013. [p, red label]'.

Current status. Valid species.

Gabrius franzi Smetana, 1967

Gabrius franzi Smetana, 1967: 153, Fig. 1.

One paratype is deposited in NMPC (ex coll. L. Hromádka):

PARATYPE (unsexed specimen): 'Valle de Lozera / lg.H.Franz [p] / [reverse of the same label] Sp 190 [hw] // Prov.Lugo / Hisp.bor. [p] // ex coll. L. Hromádka / National Museum / Prague, Czech Republic [p] // 1967 [hw] / Smetana det. [p, red label] // PARATYPUS [p] / Gabrius / franzi m. [hw, red label]'.

Current status. Valid species, see SchüLKE \& SMEtANA (2015).

Gabrius fulmarus Hromádka, 2014

Gabrius fulmarus Hromádka, 2014f: 31, Figs 17-19.

The holotype is deposited in NMPC (general collection):

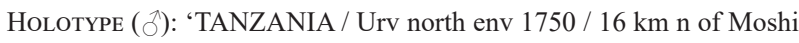
/ M. Kuboň leg. [p, yellow label] // ex coll. L. Hromádka / National Museum / Prague, Czech Republic [p] // HOLOTYPUS / GABRIUS Stephens / fulmarus sp. nov. / Hromádka det., 2014. [p, red label]’.

Current status. Valid species.

Gabrius glareola Hromádka, 2014

Gabrius glareola Hromádka, 2014f: 32, Figs 20-22.

One paratype is deposited in NMPC (ex coll. L. Hromádka): PARATYPe (đ): 'BOTSWANA (B 11) / Moremi Reservs / $19^{\circ} 23^{\prime} \mathrm{S} 23^{\circ} 33 \mathrm{E}$ / 18-20. 1972 [p] // ex coll. L. Hromádka / National Museum/Prague, Czech Republic [p] // PARATY PUS / GABRIUS Stephens / glareola sp. nov. / Hromádka, det., 2014. [p, orange label]'.

Current status. Valid species. 
Gabrius halcyon Hromádka, 2014

Gabrius halcyon Hromádka, 2014h: 178, Figs 22-24.

The holotype is deposited in NMPC (general collection): HoLотуре ( $\left.\delta^{\jmath}\right)$ : ‘ $\delta$ [p, pink label] // République Centraafricante [sic!] / Bozo lumiere / 21.v.1981. / leg. N. Degallier [p] // ex coll. L. Hromádka / National Museum / Prague, Czech Republic [p] // HOLOTYPUS / GABRIUS Stephens / halcyon sp. nov. / Hromádka, det. 2012. [p, red label]'.

Current status. Valid species.

\section{Gabrius halobaena Hromádka, 2014}

Gabrius halobaena Hromádka, 2014h: 180, Figs 28-30.

Two paratypes are deposited in NMPC (ex coll. L. Hromádka):

PARATYPE (ð): 'Massif Ruwenzori / Kyandolire 1.750 m / Riv. Mulaku / af. Kakalari (terreau) [p, yellow label] // Congo Belge / 29.iv.1954 / P. Vanschuytbroeck \& / at Kakalari (terreau) [p, yellow label] // ex coll. L. Hromádka / National Museum / Prague, Czech Republic [p] // PARATYPUS / GABRIUS Stephens / halobaena sp. nov. / Hromádka, det. 2014. [p, red label]'.

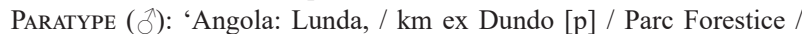
19.X.I.1972 [hw] // Exclosed / alate / emergence / silrs Nist / T-466 [hw] // coll. / O.H,AH.K.Siner / NR.J.SWIST / NO.2621 [hw] // ex coll. L. Hromádka / National Museum / Prague, Czech Republic [p] // PARATYPUS / GABRIUS Stephens / halobaena sp. nov. / Hromádka, det. 2014. [p, red label]'.

Current status. Valid species.

\section{Gabrius helogale Hromádka, 2014}

Gabrius helogale Hromádka, 2014g: 45, Figs 24-26.

The holotype is deposited in NMPC (general collection): HоLотуре (đ): 'Ethiopia: / Kulumsa River / elfiske vid vägen / 7.XII 1967 [sic!] / leg. P. Brinck et.al [p] // ex coll. L. Hromádka / National Museum / Prague, Czech Republic [p] // HOLOTYPUS / GABRIUS Step. / helogale sp. nov. / Hromádka det., 2013 [p, orange label]'.

Current status. Valid species.

Gabrius hirundo Hromádka, 2014

Gabrius hirundo Hromádka, 2014g: 47, Figs 27-29.

Two paratypes are deposited in NMPC (ex coll. L. Hromádka):

PARATYPe (đ): 'Massif Ruwenzori / Katonge $2.218 \mathrm{~m}$ [sic!] / (terreau) [p] // Congo Belge: P.N.A. / 2.-3.ii.1953 / P. Vanschuytbroeck \& / J.Kakenbosch [p] // ex coll. L. Hromádka / National Museum/Prague, Czech Republic [p] // PARATY PUS / GABRIUS Step. / hirundo sp. nov. / Hromádka, det. 2013. [p, red label]'

PARATYPe ( ): 'Massif Ruwenzori / Katonge $2.218 \mathrm{~m}$ [sic!] / (terreau) [p] // Congo Belge: P.N.A. / 2.-3.ii.1953 / P. Vanschuytbroeck \& / J.Kakenbosch [p] // ex coll. L. Hromádka / National Museum / Prague, Czech Republic [p] // PARATY PUS / GABRIUS Step. / hirundo sp. nov. / Hromádka, det. 2013. [p, orange label]'.

Current status. Valid species.

Gabrius hoberlandti Smetana, 1953

Gabrius hoberlandti Smetana, 1953b: 122, Fig. 1.

The holotype is deposited in NMPC (general collection): HoLotype ( $\left.{ }^{\jmath}\right)$ : ' $[\mathrm{hw}]$ // Ulukisla / Anat. 28. VII. 47. / Exp N Mus ČRR [sic!] [p] // HOLOTYPE [p, red label] // Gabrius Steph. [p]
/ hoberlandti / m. [hw] / det. Smetana 19 [p] 51 [hw] // Mus. Nat. Pragae / Inv. [p] 18795 [hw, orange label]’.

Current status. Valid species, see Schülke \& SMEtANA (2015).

\section{Gabrius kobayashii Smetana, 1984}

Gabrius kobayashii Smetana, 1984: 128, Figs 7-9.

One paratype is deposited in NMPC (ex coll. L. Hromádka): PARATYPe (unsexed specimen): 'JAPAN Gumma Pr. / 6km E Usui Pass / 750m 20.VII.80 / A.\&Z. Smetana [p] // ex coll. L. Hromádka / National Museum / Prague, Czech Republic [p] // PARATYPE / Gabrius kobayashii / A.Smetana [p, yellow label]'.

Current status. Valid species, see SCHÜlKE \& SMETANA (2015).

Gabrius meridies Smetana, 1995

Gabrius meridies Smetana, 1995: 668, Figs 1133-1139.

Two paratypes are deposited in NMPC (ex coll. L. Hromádka):

Paratypes (1 1 , 1 unsexed specimen): 'ARIZ. 2 mi. W Black / Lk.,Sitgreaves N.F. / VII-13-1976,2240m. / J.M. Campbell [p] // ex coll. L. Hromádka / National Museum / Prague, Czech Republic [p] // PARATYPE / Gabrius / meridies / A. Smetana 1993 / CNC No.21999 [p, yellow label]'.

Current status. Valid species, see HERMAN (2001b).

\section{Gabrius mungos Hromádka, 2014}

Gabrius mungos Hromádka, 2014i: 200, Figs 13-15.

The holotype is deposited in NMPC (general collection): HoLOTYPe (さ): 'SÜDAFRIKA,NE-Prov. / Tshipise [sic!], Honet Nature / Reserve, Camp, LF / 22³6'S, 30¹0'E, 300m / NN,23.-25.XI. 1996 / leg. M. Hartmann [p] // ex coll. L. Hromádka / National Museum / Prague, Czech Republic [p] // HOLOTYPUS / GABRIUS Step. mungos sp. nov. / Hromádka, det., 2014 [p, red label]'.

Current status. Valid species.

\section{Gabrius nepos Smetana, 1984}

Gabrius nepos Smetana, 1984: 146, Figs 41-43.

One paratype is deposited in NMPC (ex coll. L. Hromádka): PARATYPE (unsexed specimen): 'JAPAN Gumma Pr. / Usui Bypass / 700m 20.VII.80 / A.\&Z. Smetana [p] // ex coll. L. Hromádka / National Museum / Prague, Czech Republic [p] // PARATYPE / Gabrius nepos / A.Smetana [p, yellow label]'.

Current status. Valid species, see Schülke \& Smetana (2015).

\section{Gabrius obenbergeri Smetana, 1953}

Gabrius obenbergeri Smetana, 1953a: 165, Figs 14, 17.

The holotype is deposited in NMPC (general collection): HoLotype ( $\precsim$ ): ' $\delta$ [hw] // Sarajevo / ... [illegible] 507 [p] // HOLOTYPE [p, red label] // Gabrius Steph. [p] / obenbergeri / m. [hw] / det. Smetana 19 [p] 52 [hw] // Mus. Nat. Pragae / Inv. [p] 18767 [hw, orange label]'.

Current status. Valid species, see Schülke \& SmEtanA (2015). 


\section{Gabrius oena Hromádka, 2014}

Gabrius oena Hromádka, 2014g: 48, Figs 30-32.

The holotype and one paratype are deposited in NMPC (general collection):

Holotype (ð): ‘ETHIOPIA:Bale [p] / 3 [hw] [sic!] km. W. of Dinshu / 0706 N.394 [p] 5 [hw] E. / 3.050 m.xii. 1971 [p] // ex coll. L. Hromádka / National Museum / Prague, Czech Republic [p] // HOLOTYPUS / GABRIUS Step. / oena sp. nov. / Hromádka, det. 2013 [p, orange label]'.

Paratype (ð): 'ETHIOPIA: Bale / 8km [sic!]. W. of Dinshu / 0706 N. 3944 E [sic!]. / 3.050m. xii. 1971 [p] // ex coll. L. Hromádka / National Museum / Prague, Czech Republic [p] // PARATY PUS / GABRIUS Step. / oena sp. nov. / Hromádka det., 2013. [p, red label]’

Current status. Valid species.

Gabrius perodicticus Hromádka, 2014

Gabrius perodicticus Hromádka, 2014e: 699, Figs 10-12.

The holotype is deposited in NMPC (ex coll. L. Hromádka):

HoLotype (ð): 'BURUNDI - NE / Ruvubu-Parc / 25./26.2.92 [sic!] / fc.ARNDT [p] // ex coll. L. Hromádka / National Museum / Prague, Czech Republic [p] // HOLOTYPUS / GABRIUS Stephens / perodicticus sp. nov. / Hromádka, det., 2013 [p, red label]'.

Current status. Valid species.

\section{Gabrius phelsuma Hromádka, 2007}

Gabrius phelsuma Hromádka, 2007: 187, Figs 1-5.

Four paratypes are deposited in NMPC (ex coll. L. Hromádka):

PARATyPe (ठ): 'Madagascar est, 1000- / 1200m, Anosibe Ifody pr. [p] // Moramanga, 31.1.-2.2. / 1993, J. Janák lgt. [p] // ex coll. L. Hromádka / National Museum / Prague, Czech Republic [p] // PARATY PUS / GABRIUS Step. / phelsuma sp.nov. / Hromádka, det. 2007 [p, red label]'.

PARATYPe (ð): 'Madagascar Est / 1100-1200m / Massiv Ambondrombe / J.Janák + P.Moravec lgt. [p] // Ikoka env. 9.-10.3. 1996 / forêt humide, tamisages / crête Amboasa, camp 1 [p] // ex coll. L. Hromádka / National Museum / Prague, Czech Republic [p] // PARATY PUS / GABRIUS Step. / phelsuma sp.nov. / Hromádka, det. 2007 [p, red label]'.

Paratype ( ): 'Madagascar Est 1300- / 1400m / Massiv Ambondrombe / J.Janák + P.Moravec lgt. [p] // Ikoka env. 11-12.3. 96 forêt / humide,bord d'un ruisseau / crête Amboasa, camp 3 [p] // ex coll L. Hromádka / National Museum / Prague, Czech Republic [p] // PARATYPUS / GABRIUS Step. / phelsuma sp.nov. / Hromádka, det. 2007 [p, red label]'.

PARATYPe ( ): 'Madagascar Est, 14.-16.1. / Rég. Ambatondrazaka, / 5km N of Didy / J. Janák lgt. 1995 [p] // 1100-1200 m, / forêt humide / bord d'un ruisseau [p] // ex coll. L. Hromádka / National Museum / Prague, Czech Republic [p] // PARATYPUS / GABRIUS Step. / phelsuma sp.nov. / Hromádka, det. 2007 [p, red label]'.

Current status. Valid species.

\section{Gabrius philo Smetana, 1984}

Gabrius philo Smetana, 1984: 140, Figs 28-30.

One paratype is deposited in NMPC (ex coll. L. Hromádka):

PARATYPe (unsexed specimen): 'JAPON NAGANO / J. E. Kogen N. Park / Shiga [p] C. [hw] 1500 m / Löbl 23.7.1980 [p] // ex coll. L. Hromádka / National Museum / Prague, Czech Republic [p] // PARATYPE [p] / Gabrius / philo [hw] / A. Smetana [p] 1982 [hw, yellow label]'.

Current status. Valid species, see Schülke \& Smetana (2015).
Gabrius platysaurus Hromádka, 2014

Gabrius platysaurus Hromádka, 2014g: 49, Figs 33-34.

The holotype is deposited in NMPC (general collection): Holotype (ð): ‘BOTSWANA(B 11)/ Moremi Reservs / 19 ²3'S $23^{\circ} 33 \mathrm{E}$ [sic!] / 18.-20. 1972 [p] - II [hw] // ex coll. L. Hromádka / National Museum / Prague, Czech Republic [p] // HOLOTYPUS / GABRIUS Steph. / platysaurus sp. nov. / Hromádka, det., 2013. [p, red label]'.

Current status. Valid species.

\section{Gabrius plegadis Hromádka, 2014}

Gabrius plegadis Hromádka, 2014g: 50, Figs 38-39.

The holotype is deposited in NMPC (ex coll. L. Hromádka): Holotype ( ${ }^{3}$ ): 'Tanzania / Uru north, env. $1750 \mathrm{~m} / 16 \mathrm{~km} \mathrm{~N}$ of Moshi.v.2010 / Milan Kuboň lgt. [p] // ex coll. L. Hromádka / National Museum / Prague, Czech Republic [p] // HOLOTYPUS / GABRIUS Stephens / plegadis sp. novp [sic!] / Hromádka det., 2014. [p, red label]'.

Current status. Valid species.

Gabrius potamogale Hromádka, 2014

Gabrius potamogale Hromádka, 2014h: 186, Figs 42-44.

One paratype is deposited in NMPC (ex coll. L. Hromádka): Paratype ( $\precsim$ ): 'TANZANIA / Urv north env 1750 / $16 \mathrm{~km}$ n of Moshi / M. Kuboň leg. [p, yellow label] // ex coll. L. Hromádka / National Museum / Prague, Czech Republic [p] // PARATY PUS / GABRIUS Stephens / potamogale sp. nov. / Hromádka, det. 2012. [p, red label]'.

Current status. Valid species.

Gabrius pternistis Hromádka, 2014

Gabrius pternistis Hromádka, 2014g: 51, Figs 35-37.

Three paratypes are deposited in NMPC (ex coll. L. Hromádka):

Paratype (o): 'South Africa, KwaZulu-Natal / Impendle, Nhlosane farm,pitfalls / 29³5'S:295'ㄹ, 1700-1900m / - 23.Nov1993 [sic!] J. Kotze coll. [p] // Ex coll. / J. Janák [p] // ex coll. L. Hromádka / National Museum / Prague, Czech Republic [p] // PARATY PUS / GABRIUS Stephens / pternistis sp. nov. / Hromádka, det., 2014. [p, red label]'.

Paratype (unsexed specimen): 'South Africa, KwaZulu-Natal / Impendle,

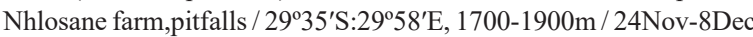
1993 [sic!], J. Kotze coll. [p] // ex coll. L. Hromádka / National Museum / Prague, Czech Republic [p] // PARATYPUS / GABRIUS Stephens / pternistis sp. nov. / Hromádka, det., 2014. [p, red label]'.

Paratype (ð): 'South Africa, KwaZulu-Natal / Impendle, Nhlosane farm,pitfalls / 29035'S:2958'E, 1700-1900m / 22Dec1993-5Jan1994 [sic!], J.Kotze coll. [p] // Ex coll. / J. Janák [p] // ex coll. L. Hromádka / National Museum / Prague, Czech Republic [p] // PARATYPUS / GABRIUS Stephens / pternistis sp. nov. / Hromádka, det., 2014. [p, red label]'.

Current status. Valid species.

Gabrius pyxicephalus Hromádka, 2014

Gabrius pyxicephalus Hromádka, 2014g: 52, Figs 40-41.

The holotype is deposited in NMPC (general collection): Holotype ( đ): ‘ANGOLA (A 25) / Rio Longs / 4 mls S. Lussusso / 8. iii. 1972 [p] // ex coll. L. Hromádka / National Museum / Prague, Czech Republic [p] // HOLOTYPUS / GABRIUS Step. / pyxicephalus sp. nov. / Hromádka, det., 2013 [p, orange label]'.

Current status. Valid species. 


\section{Gabrius robustus Smetana, 1953}

Gabrius robustus Smetana, 1953a: 168, Figs 6, 8.

The holotype is deposited in NMPC (general collection):

Holotype (ठ): ‘ $\delta$ [hw] // Kistac / maced. [hw] // HOLOTYPE [p, red label] // Gabrius Steph. [p] / robustus / m. [hw] / det. Smetana 19 [p] 52 [hw] // Mus. Nat. Pragae / Inv. [p] 18796 [hw, orange label]'.

Current status. Valid species, see Schillhammer (2009) and SchüLKE \& SMETANA (2015).

\section{Gabrius rousettus Hromádka, 2014}

Gabrius rousettus Hromádka, 2014h: 187, Figs 45-47.

The holotype and one paratype are deposited in NMPC (general collection and ex coll. L. Hromádka):

Holotype (ठ): ‘TANZANIA / Mwanza / 11.x.1969 / Ardö leg. [p] // ex coll. L. Hromádka / National Museum / Prague, Czech Republic [p] // HOLOTY PUS / GABRIUS Stephens / rousettus sp. nov. / Hromádka, det., 2012. [p, red label]'.

PARATYPe (ð): ‘TANZANIA / Mwanza / 11.x.1969 / Ardö leg. [p] // ex coll. L. Hromádka / National Museum / Prague, Czech Republic [p] // PARATY PUS / GABRIUS Stephens / rousettus sp. nov. / Hromádka, det., 2013 [p, red label]'.

Current status. Valid species.

\section{Gabrius serinus Hromádka, 2014}

Gabrius serinus Hromádka, 2014e: 699, Figs 13-15.

The holotype is deposited in NMPC (ex coll. L. Hromádka):

Holotype ( $\precsim$ ): ‘ $\delta$ [p, pink label] // Tanzania / Uru north, env. 1750 m / 16 km N of Moshi.v.2010 / Milan Kuboň lgt. [p] // ex coll. L. Hromádka / National Museum / Prague, Czech Republic [p] // HOLTYPUS [sic!] / GABRIUS Steph. / serinus sp. nov. / Hromádka, det., 2013. [p, red label]'

Current status. Valid species.

\section{Gabrius spurius Smetana, 1954}

Gabrius spurius Smetana, 1954b: 178, Figs 1-3.

Eighteen paralectotypes are deposited in NMPC (general collection):

Paralectotype ( + ): 'Ulukisla / Anat. 28. VII 47. / Exp. N Mus. ČSR [p] // ALLOTYPE [p, red label] // Gabrius Steph. [p] / spurius m / allotype 54 [hw] / det. Smetana 19 [p] // Mus. Nat. Pragae [p] / 18779 [hw] / Inv. [p, orange label]'.

Paralectotype (o): 'Ulukisla / Anat. 28. VII 47. / Exp. N Mus. ČSR [p] // PARATYPE [p, red label] // Gabrius Steph. [p] / spurius m. / paratype $54[\mathrm{hw}] /$ det. Smetana 19 [p]'.

Paralectotype ( + ): 'Ulukisla / Anat. 28. VII 47. / Exp. N Mus. ČSR [p] // PARATYPE [p, red label] // Gabrius Steph. [p] / spurius m. / paratype 54 [hw] / det. Smetana 19 [p] // Mus. Nat. Pragae [p] / 18 787 [hw] / Inv. [p, orange label]'.

Paralectotype (q): 'Ulukisla / Anat. 28. VII 47. / Exp. N Mus. ČSR [p] // PARATYPE [p, red label] // Gabrius Steph. [p] / spurius m. / paratype $54[\mathrm{hw}] /$ det. Smetana 19 [p] // Mus. Nat. Pragae [p] / 18 788 [hw] / Inv. [p, orange label]'.

Paralectotype ( + ): 'Ulukisla / Anat. 28. VII 47. / Exp. N Mus. ČSR [p] // PARATYPE [p, red label] // Gabrius Steph. [p] / spurius m. / paratype $54[\mathrm{hw}] /$ det. Smetana 19 [p] // Mus. Nat. Pragae [p] / 18 789 [hw] / Inv. [p, orange label]'.

Paralectotype ( + ): 'Ulukisla / Anat. 28. VII 47. / Exp. N Mus. ČSR [p] // PARATYPE [p, red label] // Gabrius Steph. [p] / spurius m. / paratype 54 [hw] / det. Smetana 19 [p] // Mus. Nat. Pragae [p] / 18
790 [hw] / Inv. [p, orange label]'.

Paralectotype (đ): 'Ulukisla / Anat. 28. VII 47. / Exp. N Mus. ČSR [p] // Gabrius Steph. [p] / subnigritu / lus Joy [hw] / det. Smetana 19 [p] 52 [hw] // PARATYPE [p, red label] // Gabrius Steph. [p] / spurius m. / paratype 54 [hw] / det. Smetana 19 [p] // Mus. Nat. Pragae [p]/ 18781 [hw] / Inv. [p, orange label]'.

Paralectotype (q): 'Bürücek, Toros / Anat. 29-31.7 47. / Exp N. Mus. ČSR [p] // PARATYPE [p, red label] // Gabrius Steph. [p] / spurius m. / paratype 54 [hw] / det. Smetana 19 [p] // Mus. Nat. Pragae [p] / 18792 [hw] / Inv. [p, orange label]'.

Paralectotype ( $q$ ): 'Erciyas, 1800 m / Anat. 24. VII. 47 / Exp. N. Mus. ČSR [p] // PARATYPE [p, red label] // Gabrius Steph. [p] / spurius m. / paratype 54 [hw] / det. Smetana 19 [p] // Mus. Nat. Pragae [p] / 18794 [hw] / Inv. [p, orange label]'.

Paralectotype ( + ): 'Yeniköy, Toros [hw] / Anat. [p] 30 VIII.47 [hw] / Exp. N. Mus. ČSR [p] // PARATYPE [p, red label] // Gabrius Steph. [p] / spurius m. / paratype 54 [hw] / det. Smetana 19 [p] // Mus. Nat. Pragae [p] / 18791 [hw] / Inv. [p, orange label]'.

PARALECTOTYPe (unsexed specimen): 'Ankara Cankaya / Anat. 2. VII 47. / Exp. N. Mus. ČSR [p] // PARATYPE [p, red label] // Gabrius Steph. [p] / spurius m. / paratype $54[\mathrm{hw}] /$ det. Smetana 19 [p]'.

Paralectotype (ð): 'Hu m.-Orsova [sic!] / Obenberger [p] // PARATYPE [p, red label] // Gabrius Steph. [p] / spurius m. / paratype 54 [hw] / det. Smetana 19 [p] // Mus. Nat. Pragae [p] / 18784 [hw] / Inv. [p, orange label]'

PARALECTOTYPE (§): 'Hu m.-Orsova [sic!] / Obenberger [p] // PARATYPE [p, red label] // Gabrius Steph. [p] / spurius m/paratype 54 [hw] / det. Smetana 19 [p]'.

Paralectotype (ठ): ‘Suflu Bulg.n. [hw] Coll. Purkyně [p] // PARATYPE [p, red label] // Gabrius Steph. [p] / spurius m. / paratype 54 [hw] / det. Smetana 19 [p] // Mus. Nat. Pragae [p] / 18782 [hw] / Inv. [p, orange label]'.

Paralectotype $(\precsim)$ : 'Moravia merid. / Mutěnice / J. Bechyně lgt. [p] // PARATYPE [p, red label] // Gabrius Steph. [p] / spurius m. / paratype 54 [hw] / det. Smetana 19 [p] // Mus. Nat. Pragae [p] / 18783 [hw] / Inv. [p, orange label]'

Paralectotype (ठ): 'Transsilvania [p] / Mehadia [hw] / Dr.J. Fleischer [p] // PARATYPE [p, red label] // Gabrius Steph. [p] / spurius m. / paratype 54 [hw] / det. Smetana 19 [p] // Mus. Nat. Pragae [p] / 18 780 [hw] / Inv. [p, orange label]'.

Paralectotype ( $)^{\prime}$ ): 'Wien,Umg. / Dr.Knirsch lg. [p] // // Gabrius Steph. [p] / spurius m. / paratype 54 [hw] / det. Smetana 19 [p] // PARATYPE $[\mathrm{p}$, red label]'.

Paralectotype (ふ̋): 'CAUCASUS / Svanetia.913 [sic!] / Dr.J.Veselý [p] // PARATYPE [p, red label] // Gabrius Steph. [p] / spurius m. / paratype 54 [hw] / det. Smetana 19 [p] // Mus. Nat. Pragae [p] / 18 $786[\mathrm{hw}] /$ Inv. [p, orange label]'.

Current status. Junior subjective synonym of Gabrius ravasinii Gridelli, 1920, see COIFFAIT (1974) and SCHÜLKE \& SMetana (2015).

Comments. SMetana (1954b) described Gabrius spurius referring to Turkish specimens mentioned in his previous paper under Gabrius subnigritulus Joy, 1913 (SMETANA 1953b), and to additional material from Czechoslovakia, Austria, Hungary, Bulgaria and Caucasus. The author also mentioned that a 'list of paratypes and more detailed description' will be published later. As no holotype is mentioned in the original description, all mentioned specimens are considered to be syntypes. Two years later, SMEtana (1956) provided a second description of the species, including specification of the holotype and paratypes. The subsequent designation of the holotype actually constitutes a lectotype designation according to Article 74.5 (ICZN 1999). Therefore, we consider all our type specimens of Gabrius spurius paralectotypes. 
Gabrius sterna Hromádka, 2014

Gabrius sterna Hromádka, 2014h: 188, Figs 51-53.

The holotype is deposited in NMPC (general collection): Holotype ( $\left.\jmath^{\prime}\right)$ ‘ $\delta$ [p, green label] // MALAWI S Jall env. / $30 \mathrm{~km}$ SE of Zamba / 26.-27.xii.2001 / J. Bezděk lgt. [p] // ex coll. L. Hromádka / National Museum / Prague, Czech Republic [p] // HOLOTYPUS / GABRIUS Stephens / sterna sp. nov. / Hromádka, det., 2014. [p, yellow label]'.

Current status. Valid species.

Gabrius sternula Hromádka, 2014

Gabrius sternula Hromádka, 2014i: 204, Figs 31-33.

One paratype is deposited in NMPC (ex coll. L. Hromádka):

Paratype (ठ): “TANZANIA 2224m / Mount Meru, Arusha N.P. / S03 ${ }^{\circ} 14^{\prime} 54^{\prime \prime}$;E36 48'21" / 2-4.iv.12 Dung Pitfall / leg. Smith,R \& Takano,H [p] // BMNH $\{\mathrm{E}\} /$ 2012-92 / 1233175 [p] // ex coll. L. Hromádka / National Museum / Prague, Czech Republic [p] // PARATYPUS / GABRIUS Stephens / sternula sp. nov. / Hromádka, det., 2014. [p, red label]'.

Current status. Valid species.

\section{Gabrius streptopelia Hromádka, 2014}

Gabrius streptopelia Hromádka, 2014h: 189, Figs 54-56.

The holotype and one paratype are deposited in NMPC (general collection and ex coll. L. Hromádka):

Holotype (ঐ): 'Liberia: / Charlesville [p] / 14.VI.1962 [hw] // Ex debris / Nest No [p] ... [hw, illegible] // ex coll. L. Hromádka / National Museum / Prague, Czech Republic [p] // HOLOTYPUS / GABRIUS Stephens / streptopelia spec. nov. / Hromádka, det., 2012. [p, yellow label]'.

Paratype (ð): 'LIBERIA / Mt. Nimba / Grassfield / 16.-25.ix. 1979 [p] // ex coll. L. Hromádka / National Museum / Prague, Czech Republic [p] // PARATYPUS / GABRIUS Stephens / streptopelia sp. nov / Hromádka det., 2013. [p, yellow label]'.

Current status. Valid species.

Gabrius taphozous Hromádka, 2011

Gabrius taphozous Hromádka, 2011f: 1391, Figs 40-42.

One paratype is deposited in NMPC (ex coll. L. Hromádka):

PARATYPe (ठ): 'Madagascar Nord / 600-700 m / Massiv Anjanaharibe Nord / sentier Ambodihasina- / Ambalarombe [p] // Riv.Andramonta env. / 24-29.2.96 / forêt humide, marais / J.Janák + P.Moravec lgt. [p] // ex coll. L. Hromádka / National Museum / Prague, Czech Republic [p] // PARATYPUS / GABRIUS Stephens / taphozous sp. nov. / Hromádka, det. 2007 [p, orange label]'.

Current status. Valid species.

\section{Gabrius vindex Smetana, 1995}

Gabrius vindex Smetana, 1995: 648, Figs 1078-1084.

One paratype is deposited in NMPC (ex coll. L. Hromádka):

PARAType (unsexed specimen): 'MAN.Riding Mtn.N.P. / Central Trail 5 km / SE Gunn Lake 14.IX. / 1979 A. Smetana [p] // Ex: beaver / lodge [p] // ex coll. L. Hromádka / National Museum / Prague, Czech Republic [p] // PARATYPE / Gabrius / vindex / A. Smetana 1993 / CNC No.21993 [p, yellow label]'.

Current status. Valid species, see Herman (2001b).

\section{Gabrius yamanei Smetana, 1984}

Gabrius yamanei Smetana, 1984: 130, Figs 13-15.

Two paratypes are deposited in NMPC (ex coll. L. Hromádka):

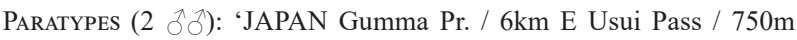
20.VII.80 / A.\&Z. Smetana [p] // ex coll. L. Hromádka / National Museum / Prague, Czech Republic [p] // PARATYPE / Gabrius yamanei / A.Smetana [p, yellow label]'.

Current status. Valid species, see SchüLKE \& SMETANA (2015).

\section{Gabronthus apaloderma Hromádka, 2014}

Gabronthus apaloderma Hromádka, 2014b: 100, Figs 1-3.

The holotype is deposited in NMPC (ex coll. L. Hromádka): HoLOTyPe ( §’): 'UGANDA CW. / Kasese, Kilembe / E Ruwenzori [sic!] 23.11. [sic!] / lgt. M. Snížek 2001 [p] // ex coll. L. Hromádka / National Museum / Prague, Czech Republic [p] // HOLOTYPUS / GABRONTHUS Tott. / apaloderma sp. nov. / Hromádka det., 2013. [p, orange label]'.

Current status. Valid species.

Gabronthus bycanistes Hromádka, 2014

Gabronthus bycanistes Hromádka, 2014b: 101, Figs 4-6.

The holotype and one paratype are deposited in NMPC (general collection and ex coll. L. Hromádka):

HoLotype (đ): 'BURUNDI / Kaninya / vii. 1940 / A. J Bréda leg. [p] // ex coll. L. Hromádka / National Museum / Prague, Czech Republic [p] // HOLOTY PUS / GABRONTHUS / bycanistes sp. nov / Hromádka, det. 2013. [p, orange label]'.

ParatyPe (đ̋): 'BURUNDI / Kaninya / vii. 1940 / A. J. Bréda leg. [p] // ex coll. L. Hromádka / National Museum / Prague, Czech Republic [p] // PARATYPUS / GABRONTHUS Tott. / bycanistes sp. nov. / Hromádka, det., 2013. [p, red label]'.

Current status. Valid species.

\section{Gabronthus corythaeola Hromádka, 2014}

Gabronthus corythaeola Hromádka, 2014b: 102, Figs 7-9.

Three paratypes are deposited in NMPC (ex coll. L. Hromádka):

Paratypes (3 unsexed specimens): ‘TANZANIA sept. / Ngare Sero, 1200 / $20 \mathrm{~km} \mathrm{E}$ of Arusha [p] // 17.II.-6.III.1982. / leg.H.J.Bremer [p] // ex coll. L. Hromádka / National Museum / Prague, Czech Republic [p] // PARATYPUS / GABRONTHUS Tott. / corythaeola sp. nov. / Hromádka det., 2013. [p, orange label]'.

Current status. Valid species.

\section{Gabronthus mandrillus Hromádka, 2014}

Gabronthus mandrillus Hromádka, 2014b: 103, Figs 10-12.

The holotype is deposited in NMPC (general collection): Holotype ( $\left.{ }^{\prime}\right)$ : 'Rep. South Africa / Northern Prov. Camp / David, $5 \mathrm{~km}$ S. Ofoolaco / 475m 17.-24.i.2002 / leg. S. Murzin [p, green label] // ex coll. L. Hromádka / National Museum / Prague, Czech Republic [p] // HOLOTYPUS / GABRONTHUS / mandrillus sp. nov / Hromádka, det. 2013. [p, orange label]'.

Current status. Valid species. 
Gabronthus mirafra Hromádka, 2014

Gabronthus mirafra Hromádka, 2014b: 104, Figs 13-15.

The holotype is deposited in NMPC (general collection):

Holotype (ð): 'KENYA, Mt. Elgon Nat.P., / near Chepnyalil Cave, / dry evergreen montane / forest, $2500 \mathrm{~m}$ [p] // singled \& swept from / the vegetation, / 24.-28.I.1992. No.507 / O.Merk1 \& G.Várkonyi [p] // ex coll. L. Hromádka / National Museum / Prague, Czech Republic [p] // HOLOTYPUS / GABRONTHUS Tott. / mirafra sp. nov. / Hromádka, det., 2013. [p, red label]'.

Current status. Valid species.

\section{Gabronthus mycteria Hromádka, 2014}

Gabronthus mycteria Hromádka, 2014b: 105, Figs 16-18.

The holotype is deposited in NMPC (general collection):

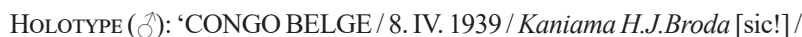
Cafe arabica [p, green label] // ex coll. L. Hromádka / National Museum / Prague, Czech Republic [p] // HOLOTYPUS / GABRONTHUS Tott. / mycteria sp. nov. / Hromádka, det., 2013. [p, red label]'.

Current status. Valid species.

\section{Gabronthus polemaetus Hromádka, 2013}

Gabronthus polemaetus Hromádka, 2013a: 73, Figs 1-2.

Four paratypes are deposited in NMPC (ex coll. L. Hromádka):

Paratypes (1 $\delta, 2$ unsexed specimens): 'REP. S. AFR.: Cape / Prov., Knysna, / Diepwalle, / 12.XII.1981 [p] // FMHD\#81-603, elephant / dung., S. Peck / FIELD MUSEUM [p] // ex coll. L. Hromádka / National Museum / Prague, Czech Republic [p] // PARATYPUS / GABRONTHUS Totten. / polemaetus sp. nov. / Hromádka, det. 2007. [p, orange label]'

Paratype (unsexed specimen): 'REP. SO. AFR.: Cape / Prov., Knysna, / Diepwalle, 12-XII-1981 [p] // FMHD\#81-603, elephant / dung. S. Peck, berlese / FIELD MUSEUM [p] // ex coll. L. Hromádka / National Museum / Prague, Czech Republic [p] // PARATY PUS / GABRONTHUS Totten. / polemaetus sp. nov. / Hromádka, det. 2007. [p, orange label]'.

Current status. Valid species.

\section{Gabronthus veselskyi Hromádka, 2016}

Gabronthus veselskyi Hromádka, 2016e: 384, Figs 4-6.

The holotype is deposited in NMPC (ex coll. L. Hromádka): HoLотуPe (ふ): 'Niger 3.11.1978 / Abargasit / R. Macek, lgt. [p] // ex coll. L. Hromádka / National Museum / Prague, Czech Republic [p] // HOLOTYPUS / GABRONTHUS / veselskyi sp. nov. / Hromádka, 2016. [p, red label]'.

Current status. Valid species.

\section{Hesperus anas Hromádka, 2009}

Hesperus anas Hromádka, 2009d: 67, Figs 1-6.

Three paratypes are deposited in NMPC (ex coll. L. Hromádka):

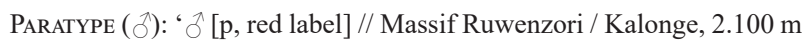
/ Riv. Nyamwamba, / aff. Butahu [p] // Congo Belge : P.N.A. / 2527-viii-1952 / P. Vanschuytbroeck \& / J. Kekenbosch 902-03 [p] // ex coll. L. Hromádka / National Museum / Prague, Czech Republic [p] // PAR ATY PUS / HESPERUS Fauvel / anas sp. n. / Hromádka det. 2008 [p, orange label]'.

PARATYPES (2 9 ) : ‘ 9 [p, orange label] // Massif Ruwenzori / Kalonge, $2.060 \mathrm{~m}$ [sic!] / Katauleko, af. Butahu [p] // Congo Belge : P.N.A. / 2-x-1952 / P. Vanschuytbroeck \& / J. Kekenbosch 1126 [p] // ex coll. L. Hromádka / National Museum / Prague, Czech Republic [p] // PARATYPUS / HESPERUS Fauvel / anas sp. n. / Hromádka det. 2008 [p, orange label]'

Current status. Trapeziderus anas (Hromádka, 2009), see Chani-Posse de Maus et al. (2018).

\section{Hesperus kuboni Hromádka, 2004}

Hesperus kuboni Hromádka, 2004: 114, Figs 1-5.

The holotype is deposited in NMPC (ex coll. L. Hromádka): Holotype (đ): 'TANZANIA, ARUSHA / 6.-7. 4.1997, Mt.Meru / 1800 2000 / M. Kuboň leg. [p] // ex coll. L. Hromádka / National Museum / Prague, Czech Republic [p] // HOLOTYPUS / PHILONTHUS (s. str.) / kuboni sp. nov. / Hromádka., det., 2003. [p, red label]'.

Current status. Philonthus kuboni (Hromádka, 2004), see HromÁDKA (2012b).

\section{Hesperus mylomys Hromádka, 2009}

Hesperus mylomys Hromádka, 2009d: 70, Figs 10-16.

One paratype is deposited in NMPC (ex coll. L. Hromádka):

PARATYPE ( $\partial$, dissected genitalia on a separate plastic card): 'R.S.Africa: 10.xi.1997 / 34²7.2'S/20²4.2'E Cape / Prov.:De Hoop NR: / Carex sievings / leg. M. Uhlig [p] // ex coll. L. Hromádka / National Museum / Prague, Czech Republic [p] // PARATY PUS / HESPERUS Fauvel / mylomys sp. nov. / Hromádka, det. 2009. [p, red label]'.

Current status. Valid species.

\section{Hesperus oriolus Hromádka, 2012}

Hesperus oriolus Hromádka, 2012b: 569, Figs 61-63.

One paratype is deposited in NMPC (ex coll. L. Hromádka): Paratype ( + ): 'Bamenda / 17.12.55 [p] // Nigeria-Kamerun / Bechyne 1955-56 [p] // ex coll. L. Hromádka / National Museum / Prague, Czech Republic [p] // PARATY PUS / HESPERUS Fauvel / oriolus sp. nov. / Hromádka, det. 2011. [p, orange label]'.

Current status. Trapeziderus oriolus (Hromádka, 2012), see Chani-Posse de Maus et al. (2018).

\section{Hesperus purpuripennis Bernhauer, 1915}

Hesperus purpuripennis Bernhauer, 1915: 189.

One syntype is deposited in NMPC (general collection): SYNTYPE (unsexed specimen): '1238 [hw] // Neu-Britannien / Ralum / F.Dahl S. [p, violet label] // Type [p, red label] // Hesperus / purpuripennis / Bernh. [hw]'.

Current status. Valid species, see Herman (2001b).

Comments. One hind wing of the syntype is mounted on a separate card.

\section{Jurecekia paradoxa Rambousek, 1920}

Jurečekia paradoxa Rambousek, 1920: 17.

Five syntypes are deposited in NMPC (general collection): SYNTYPE (đ): 'Tockoe Ros. or. / Gub. Samara / Dr.Jureček 1916 [p] // TYPE [p, red label] // Jurečekia Ramb. / asphaltinus Er. / = / paradoxa Ramb. [hw]'.

SyNTYPE (unsexed specimen): ‘Tockoe Ros. or. / Gub. Samara / Dr.Jureček 1916 [p] // TYPUS [p, red label] // Jurečekia / paradoxa / mihi [hw] / det.Rambousek [p] // Mus. Nat. Pragae / Inv. [p] 19396 [hw, orange label]'. 
SyntyPe ( + ): ‘ $\bigcirc$ [hw] // Tockoe Ros. or. / Gub. Samara / Dr.Jureček [p] // TYPUS [p, red label] // Jurečekia / paradoxa m. [hw] / det.Dr.Rambousek [p] // Mus. Nat. Pragae / Inv. [p] 19395 [hw, orange label]'.

SyntyPe ( + ): ‘ $q$ [hw] // Tockoe Ros. or. / Gub. Samara / Dr.Jureček 1916 [p] // TYPUS [p, red label] // Jurečekia / paradoxa m. [hw] / det.Rambousek [p] // Mus. Nat. Pragae / Inv. [p] 19394 [hw, orange label]'.

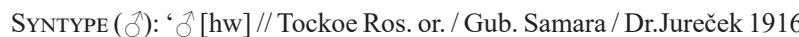
[p] // TYPUS [p, red label] // Jurečekia / paradoxa m. [hw] / det.Rambousek [p] // Mus. Nat. Pragae / Inv. [p] 19393 [hw, orange label]'.

Current status. Junior subjective synonym of Jurecekia asphaltina (Erichson, 1840), see RAMBOUSEK (1923) and SCHÜLKE \& SMETANA (2015).

Mentophilonthus crocuta Hromádka, 2009

Mentophilonthus crocuta Hromádka, 2009b: 124, Figs 11-15.

The holotype is deposited in NMPC (general collection):

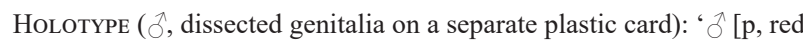
label] // GHANA / MKAWKAW E ... [illegible] / 20 IV 84 Leg. W. ROSSI [p] // ex coll. L. Hromádka / National Museum/ Prague, Czech Republic [p] // HOLOTYPUS / MENTOPHILONTHUS / crocuta sp. n. $\delta$ / L. Hromádka, det. 2005 [p, red label]'.

Current status. Philonthoblerius crocuta (Hromádka, 2009), see NeWTON (2015).

\section{Mentophilonthus davidkrali Hromádka \& Schillhammer, 2007}

Mentophilonthus davidkrali Hromádka \& Schillhammer, 2007: 2, Figs $2,4,6,9$.

The holotype is deposited in NMPC (ex coll. L. Hromádka):

НоLотуре ( $\partial$, dissected genitalia on a separate plastic card): ' $\delta[\mathrm{p}$, pink label] // E-NEPAL 6-10/7.2000. / Kangchenjunga Himal Mts. / GHUNSA vill. 3375m / 27.40N 87.56E [GPS] env., / David Král lgt. [p] // NEPAL Expedition, / Jan Farkač, David Král / \& Jan Schneider, 2000 [p] // ex coll. L. Hromádka / National Museum / Prague, Czech Republic [p] // HOLOTYPUS / MENTOPHILONTHUS / davidkrali sp. n. / Hromádka \& / Schillhammer, 2005. [p, red label]'.

Current status. Philonthoblerius davidkrali (Hromádka \& Schillhammer, 2007), see NEWTON (2015).

Mentophilonthus equus Hromádka, 2009

Mentophilonthus equus Hromádka, 2009b: 132, Figs 41-42.

The holotype is deposited in NMPC (general collection): Holotype (q): 'MALAWI S Masenjere / $80 \mathrm{~km} \mathrm{~S}$ of Blantyse / 21.-22. xii. 2001 / J. Bezděk, lgt. [p, yellow label] // ex coll. L. Hromádka / National Museum / Prague, Czech Republic [p] // HOLOTYPUS / MENTOPHILONTHUS / equus sp. n. / Hromádka,det.2005 [p, red label]'.

Current status. Philonthoblerius equus (Hromádka, 2009), see NeWTON (2015).

\section{Mentophilonthus hystrix Hromádka, 2009}

Mentophilonthus hystrix Hromádka, 2009b: 134, Figs 43-48.

Five paratypes are deposited in NMPC (ex coll. L. Hromádka):

PARATYPes (2 え): 'S.W.Afr., Damaral. / Grootberg Pass / 19.55S - 13.59E [p] // 16.2. 1975; E-Y:669 / elephant dung / leg. Endrödy-Younga [p] // ex coll. L. Hromádka / National Museum / Prague, Czech Republic [p] // PARATYPUS / Philonthus (s. str.) / hystrix sp. n. / L. Hromádka, det.2004 [p, orange label]'.
Paratypes (2 $\delta$ ) : 'S.W.Afr., Damaral. / Grootberg Pass / 19.55S - 13.59E [p] // 16.2. 1975; E-Y:669 / elephant dung / leg. Endrödy-Younga [p] // ex coll. L. Hromádka / National Museum / Prague, Czech Republic [p] // PARATYPUS / MENTOPHILONTHUS / hystrix sp. n. o / Hromádka, det. 2005 [p, orange label]'.

PARATYPe ( $q$ ): ‘ $q$ [p, orange label] // S.W.Afr., Damaral. / Grootberg Pass / 19.55S - 13.59E [p] // 16.2. 1975. E-Y:669 / elephant dung / leg. Endrödy-Younga [p] // ex coll. L. Hromádka / National Museum / Prague, Czech Republic [p] // PARATYPUS / MENTOPHILONTHUS / hystrix sp. n. + / L. Hromádka, det.2005 [p, orange label]’.

Current status. Philonthoblerius hystrix (Hromádka, 2009), see NewTON (2015).

\section{Mentophilonthus manis Hromádka, 2009}

Mentophilonthus manis Hromádka, 2009b:138, Figs 54-55.

The holotype is deposited in NMPC (general collection): HoLOTYPe ( $\uparrow): ~ ‘ q[p$, orange label] // GABON, Belinga / 12.III.1963 (199 L) / Leg. H. Coiffait [p, blue label] // ex coll. L. Hromádka / National Museum / Prague, Czech Republic [p] // HOLOTYPUS / MENTOPHILONTHUS / manis sp. nov. / Hromádka, det. 2007 [p, orange label]'.

Current status. Philonthoblerius manis (Hromádka, 2009), see NewTon (2015).

\section{Mentophilonthus spermophaga Hromádka, 2012}

Mentophilonthus spermophaga Hromádka, 2012a: 1156, Figs 1-5.

The holotype is deposited in NMPC (ex coll. L. Hromádka): HoLotype (§): 'République Centraafricante [sic!] / Bozo lumiere / 21. v. 1981 / leg. N. Degallier [p] // ex coll. L. Hromádka / National Museum / Prague, Czech Republic [p] // HOLOTYPUS / MENTOPHILONTHUS / spermophaga sp. nov. / Hromádka, det., 2012. [p, red label]'.

Current status. Philonthoblerius spermophaga (Hromádka, 2012), see Newton (2015).

\section{Mentophilonthus struthio Hromádka, 2009}

Mentophilonthus struthio Hromádka, 2009b: 150, Figs 95-102.

The holotype and four paratypes are deposited in NMPC (general collection and ex coll. L. Hromádka):

HoLotyPe (đ): ‘ ‘ [p, pink label] // SUDAN mer. / Shambe / 15. 10. 1966 / Dr. P. Štys leg. [p, green label] // ex coll. L. Hromádka / National Museum / Prague, Czech Republic [p] // HOLOTYPUS / MENTOPHILONTHUS / struthio sp. nov. / Hromádka, det. 2007 [p, orange label]'.

ParatyPes (1 1 \%): 'Massif Ruwenzori / riv. Kakalari, / affl. Bombi, 1.725 $\mathrm{m}[\mathrm{p}]$ // Congo Belge : P.N.A. / 12-VI-1954 / P. Vanschuytbroeck \& / H. Synave 8905-08 [sic!] [p] // ex coll. L. Hromádka / National Museum/ Prague, Czech Republic [p] // PARATYPUS / MENTOPHILONTHUS / struthio sp. nov. / Hromádka, det. 2007 [p, orange label]'.

PARATYPE ( (đ): 'Massif Ruwenzori / Grotte Ibatama / 1.610 m, (lumière) [p] // Congo Belge : P.N.A. / 4-V-1958 / P. Vanschuytbroeck / VS377 [p] // ex coll. L. Hromádka / National Museum / Prague, Czech Republic [p] // PARATYPUS / MENTOPHILONTHUS / struthio sp. nov. / Hromádka, det. 2007 [p, orange label]'.

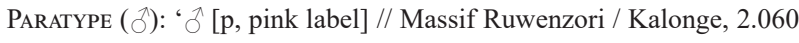
$\mathrm{m} /$ riv. Katauleko, / affl. Butahu [p] // Congo Belge : P.N.A. / 9-XII1957 / P. Vanschuytbroeck / VS 255 [p] // ex coll. L. Hromádka / National Museum / Prague, Czech Republic [p] // PARATYPUS / MENTOPHILONTHUS / struthio sp. nov. / Hromádka, det. 2007 [p, orange label]'.

Current status. Philonthoblerius struthio (Hromádka, 2009), see Newton (2015). 
Neobisnius parcepunctatus Bernhauer, 1912

Neobisnius parcepunctatus Bernhauer, 1912: 176.

Two syntypes are deposited in NMPC (general collection):

SynTyPe (unsexed specimen): 'Chaunar Reg. / Arg. lg. Jensen [hw] // parcepunctatus / Bernh. Cotypus [hw]'.

SynTYPE (unsexed specimen): 'Chaunar Reg. / Arg. lg. Jensen [hw] // parcepunctatus / Brh. Cotypus [hw]'.

Current status. Valid species, see HERMAN (2001b).

\section{Philonthus (Gabrius) kuanshanensis Bernhauer, 1914}

Philonthus (Gabrius) kuanshanensis Bernhauer, 1914: 66.

One syntype is deposited in NMPC (general collection):

SYNTYPE (unsexed specimen): 'Chikuanshan / S.Mandschur [p] // kuandschanensis / cotype Brh. [hw] // COTYPUS [p, red label] // Gabrius [p] / kuanshanensis / Bernh. 94 [hw] / L.Hromádka det. [p]'.

Current status. Gabrius kuanshanensis (Bernhauer, 1914), see Schülke \& SMetana (2015).

Philonthus (Gabrius) mandschuricus Bernhauer, 1914 Philonthus (Gabrius) mandschuricus Bernhauer, 1914: 66.

One syntype is deposited in NMPC (general collection):

SyntyPe (unsexed specimen): 'Chikuanshan / S.Mandschur [p] // Ph. mandschuricus / cotype Bernh. [hw] // Gabrius [p] / mandschuricus / Bernh. 94 [hw] / L.Hromádka det. [p]'.

Current status. Gabrius mandschuricus (Bernhauer, 1914), see Schülke \& SMetana (2015).

\section{Philonthus accipiter Hromádka, 2011}

Philonthus accipiter Hromádka, 2011c: 152, Figs 1-4.

The holotype and three paratypes are deposited in NMPC (ex coll. L. Hromádka):

Holotype (ð): ‘KENYA / M. Elgon 2380 m. / Reinhardt leg. [p] // ex coll. L. Hromádka / National Museum / Prague, Czech Republic [p] // HOLOTY PUS / PHILONTHUS (s. str.) / accipiter sp. nov. / Hromádka, det. 2009. [p, orange label]'.

Paratypes (2 ô, 1 unsexed specimen): 'KENYA / M. Elgon 2380 m. / Reinhardt leg. [p] // ex coll. L. Hromádka / National Museum / Prague, Czech Republic [p] // PARATYPUS / PHILONTHUS (s. str.) / accipiter sp. nov. / Hromádka, det. 2009. [p, orange label]'.

Current status. Valid species.

\section{Philonthus acinonyx Hromádka, 2010}

Philonthus acinonyx Hromádka, 2010e: 5, Figs 2-8.

Two paratypes are deposited in NMPC (ex coll. L. Hromádka):

PARATYPe ( $\hat{\partial}$, dissected genitalia on a separate plastic card): 'NIGERIA - Africa / UMUDIKE / 14.-27.ix. 1960 / J.M. Gregory [sic!] [p] // 3.June.1994 / Coll. W. Rossi [p] // ex coll. L. Hromádka / National Museum / Prague, Czech Republic [p] // PARATYPUS / PHILONTHUS (Pseudophil.) / acinonyx spec. nov. / Hromádka, det. 2006. [p, red label]'.

PARATYPE ( + ): ‘ $q[\mathrm{p}$, orange label] // NIGERIA - Africa / UMUDIKE / 14.-27.ix. 1960 / J.M. Gregory [sic!] [p] // ex coll. L. Hromádka / National Museum / Prague, Czech Republic [p] // Desiderata / ex.NHM / 3.iii.2005 [p] // PARATY PUS / PHILONTHUS (Pseudophil.) / acinonyx spec. nov. / Hromádka, det. 2006. [p, red label]'.

Current status. Valid species.
Philonthus actophilornis Hromádka, 2011

Philonthus actophilornis Hromádka, 2011b: 38, Figs 1-4.

The holotype is deposited in NMPC (general collection):

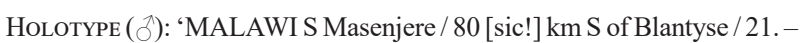
22., xii. 2001 / J. Bezděk, lgt. [p, yellow label] // ex coll. L. Hromádka / National Museum / Prague, Czech Republic [p] // HOLOTYPUS / PHILONTHUS (s. str.) / actophilornis sp. nov. / Hromádka, det. 2009. [p, orange label]'.

Current status. Valid species.

Philonthus aepyceros Hromádka, 2012

Philonthus aepyceros Hromádka, 2012c: 137, Figs 1-3.

The holotype and one paratype are deposited in NMPC (general collection and ex coll. L. Hromádka):

HoLotype (ð): ‘RWANDA / Urundi 2117 / Kisosi [sic!] [p] // ex coll. L. Hromádka / National Museum / Prague, Czech Republic [p] // HOLOTY PUS / PHILONTHUS (s. str.) / aepyceros sp.nov. / Hromádka, det. 2010. [p, orange label]'.

PARATYPE (ð): ‘RWANDA/ Nyakabuye [sic!] / 5.7.1983 / leg.H.Mühle [p] // ex coll. L. Hromádka / National Museum / Prague, Czech Republic [p] // PARATYPUS / PHILONTHUS (s. str.) / aepyceros sp.nov. / Hromádka, det. 2010. [p, orange label]'.

Current status. Valid species.

\section{Philonthus agapornis Hromádka, 2013}

Philonthus agapornis Hromádka, 2013b: 2, Figs 1-4.

One paratype is deposited in NMPC (ex coll. L. Hromádka):

PARATYPE (đ): 'Soil-Zoological Exp. / Congo-Brazzaville / Lefinie reservation / Nambouli river [p] // 12.1.1964. No 658 / sifted and singled / from elephant excr. / leg.Endrödy-Younga [p] // ex coll. L. Hromádka / National Museum / Prague, Czech Republic [p] // PARATYPUS / PHILONTHUS (s. str.) / agapornis sp. nov. / Hromádka, det. 2012. [p, red label]'.

Current status. Valid species.

Philonthus alcelaphus Hromádka, 2011

Philonthus alcelaphus Hromádka, 2011c: 156, Figs 5-8.

The holotype is deposited in NMPC (general collection): HoLOtype (ð): 'ETHIOPIA: Bale / 8km. W. of Dinshu / 0706 N. 3444 E. / 3.050m. xii. 1971 [p] // ex coll. L. Hromádka / National Museum / Prague, Czech Republic [p] // HOLOTY PUS / PHILONTHUS (s. str.) / alcelaphus sp. nov. / Hromádka, det. 2009. [p, orange label]'.

Current status. Valid species.

\section{Philonthus alesi Hromádka, 2011}

Philonthus alesi Hromádka, 2011a: 191, Figs 5-8.

The holotype is deposited in NMPC (ex coll. L. Hromádka): HoLotype (đ)): 'ETHIOPIA/ Bahr - Dar / 4. 6. 1967 / P. Štys leg. [p] // ex coll. L. Hromádka / National Museum / Prague, Czech Republic [p] // HOLOTYPE / PHILONTHUS (s. str.) / alesi sp. nov. / Hromádka, det., 2011. [p, red label]'.

Current status. Valid species.

Philonthus amandava Hromádka, 2013

Philonthus amandava Hromádka, 2013d: 385, Figs 15-17.

The holotype and one paratype are deposited in NMPC (general collection and ex coll. L. Hromádka): 
Holotype (§) ): ‘ABYSSINA / Gama Prov. / between Dita and / Bonghé, c. 9000 - / 10,5000 ft. 5.xii.1948 [p] // ex coll. L. Hromádka / National Museum / Prague, Czech Republic [p] // HOLOTYPUS / PHILONTHUS Stephens / amandava sp. nov. / Hromádka, det. 2010 [p, orange label]'.

Paratype ( + ): 'ABYSSINA / Gama Prov. / between Dita and / Bonghé, c. 9000 - / 10,5000 ft. 5.xii.1948 [p] // ex coll. L. Hromádka / National Museum / Prague, Czech Republic [p] // PARATYPUS / PHILONTHUS Stephens / amandava sp. nov. / Hromádka, det. 2010 [p, orange label]'.

Current status. Valid species.

Philonthus antidorcas Hromádka, 2010

Philonthus antidorcas Hromádka, 2010e: 10, Figs 16-19.

The holotype is deposited in NMPC (general collection): Holotype (ठ): 'MALAWI S. Jall env. / 30km SE of Zomba / 26.-27. xii.2001 / J. Bezděk, lgt. [p, yellow label] // ex coll. L. Hromádka / National Museum / Prague, Czech Republic [p] // HOLOTYPUS / PHILONTHUS (Pseudoph.) / antidorcas sp. nov. / Hromádka, det. $200[\mathrm{p}] 8$ [hw, red label]'.

Current status. Valid species.

\section{Philonthus aquila Hromádka, 2011}

Philonthus aquila Hromádka, 2011b: 40, Figs 5-8.

The holotype is deposited in NMPC (general collection): HоLотуре ( $)$ : ‘ $\delta$ [p, pink label] // CONGO BELGE / Libenge / 29. 4. 1948 / R.Cremer - M. Neuman [p] // ex coll. L. Hromádka / National Museum / Prague, Czech Republic [p] // HOLOTYPUS / PHILONTHUS (s. str.) / aquila sp. nov. / Hromádka, det. 2009. [p, orange label]'.

Current status. Valid species.

\section{Philonthus ardeotis Hromádka, 2010}

Philonthus ardeotis Hromádka, 2010e: 12, Figs 20-23.

Four paratypes are deposited in NMPC (ex coll. L. Hromádka):

ParatyPe ( $\precsim$ ): ‘ $₫$ [p, green label] // Kenya: Mt. Kenya / Theiba Forest 8000' / 2-3.V.1969 [p] // ex debris / piles / Nest No. 135 [p] // Coll. G.R. / Cunningham- / Van Someren / No. 1688 [p] // ex coll. L. Hromádka / National Museum / Prague, Czech Republic [p] // PARATY PUS / PHILONTHUS / sg. Pseudophilonthus / ardeotis sp. nov. / Hromádka, det. 2006 [p, orange label]'.

ParatyPe (đ)): ‘Kenya: Mt. Kenya / Theiba Forest 8000 / 2-3.V.1969 [p] // ex debris / piles / Nest No. 135 [p] // Coll. G.R. / Cunningham- / Van Someren / No. 1688 [p] // ex coll. L. Hromádka / National Museum / Prague, Czech Republic [p] // PARATYPUS / PHILONTHUS / sg. Pseudophilonthus / ardeotis sp. nov. / Hromádka, det. 2006 [p, orange label]'.

ParatYPe (ð): ‘TANZANIA: Tanga / Distr., Amani / 21.IV.1966 [sic!] [p] // ex debris / piles / Nest No. 122 [p] // Coll. D.H.,A.C., / \& A.H. Kistner / Field No.14 [sic!] [p] 92 [hw] // ex coll. L. Hromádka / National Museum / Prague, Czech Republic [p] // PAR ATY PUS / PHILONTHUS / sg. Pseudophilonthus / ardeotis sp. nov. / Hromádka, det. 2006 [p, orange label]'.

ParatyPe (unsexed specimen): 'Kenya: Karen / V: 1962 [p] // ex debris pile / Nest No. [p] 89 [hw] // Coll. G. R. / Cunningham- / Van Someren / Field No. [p] 968d [sic!] [hw] // ex coll. L. Hromádka / National Museum / Prague, Czech Republic [p] // PARATY PUS / PHILONTHUS / sg.Pseudophilonthus / ardeotis sp. nov. / Hromádka, det. 2006 [p, orange label]'.

Current status. Valid species.
Philonthus arvicanthis Hromádka, 2011

Philonthus arvicanthis Hromádka, 2011b: 41, Figs 9-12.

The holotype is deposited in NMPC (general collection): HoLOtype (đ): 'Kundelungu 1750 m [p] / 30-III- [hw] 1950 [sic!] / N. Leleup [p] / (Prairie marécageuse) [hw] // Récolté dans / nid de / Cryptomys [p, green label] // ex coll. L. Hromádka / National Museum / Prague, Czech Republic [p] // HOLOTY PUS / PHILONTHUS (s. str.) / arvicanthis sp. nov. / Hromádka, det. 2009. [p, orange label]'.

Current status. Valid species.

Philonthus asio Hromádka, 2013

Philonthus asio Hromádka, 2013b: 3, Figs 5-7.

The holotype is deposited in NMPC (general collection): HoLOtype (ð): '26./27.11.2001 [sic!] RSA / KwaZulu/Natal Umzinto / Vernon Crookes NR / P. Schüle leg. [p] // ex coll. L. Hromádka / National Museum / Prague, Czech Republic [p] // HOLOTYPUS / PHILONTHUS (s. str.) / asio sp.nov. / Hromádka, det-., 2012 [p, red label]'.

Current status. Valid species.

Philonthus atherurus Hromádka, 2012

Philonthus atherurus Hromádka, 2012c: 138, Figs 4-7.

One paratype is deposited in NMPC (ex coll. L. Hromádka): PARATYPE (đ): 'I.R.S.A.C.-MUS.CONGO / Kibali-Ituri: Terr. Djugu, / Mt. Aboro, 2200 m. / N.Leleup I-1954 [p] // Dans humus / en forêt [p, blue label] // ex coll. L. Hromádka / National Museum / Prague, Czech Republic [p] // PARATY PUS / PHILONTHUS (s. str.) / atherurus sp.nov. / Hromádka, det. 2010. [p, orange label]'.

Current status. Valid species.

Philonthus balearica Hromádka, 2010

Philonthus balearica Hromádka, 2010e: 14, Figs 31-36.

One paratype is deposited in NMPC (ex coll. L. Hromádka): ParatyPe (+): 'LIBERIA: / Mt.Nimba. / Grassfield, / 16-25.ix.1979. [p] // In human / faeces [p] // lowland / forest $500 \mathrm{~m}$. [p] // ex coll. L. Hromádka / National Museum / Prague, Czech Republic [p] // PARATYPUS / PHILONTHUS / sg. Pseudophilonthus / balearica sp. nov. / Hromádka, det. 2006 [p, orange label]'.

Current status. Valid species.

Philonthus barbarae Hromádka, 1992

Philonthus [s.str.] barbarae Hromádka, 1992: 98, Figs 3-5.

The holotype is deposited in NMPC (ex coll. L. Hromádka): HoLOtyPe (ð): 'NEPAL-Tanje / 24.9.1980 / J. SEIFERT [hw] // ex coll. L. Hromádka / National Museum / Prague, Czech Republic [p] // HOLOTY PUS / PHILONTHUS (s. str.) / barbarae sp. nov. / Hromádka, det. 1992. [p, orange label] // Philonthus [p] / riparius CAM. [hw] / det. Schillhammer 200 [p] 7 [hw]'.

Current status. Valid species, see SchüLKE \& SMETANA (2015); the synonymy with Philonthus riparius Cameron, 1926 indicated by Schillhammer has never been published.

\section{Philonthus batis Hromádka, 2016}

Philonthus batis Hromádka, 2016c: 372, Figs 1-3.

The holotype is deposited in NMPC (general collection): Holotype (ð): ‘TANZANIA / Mwanza / 11.x.1969 [sic!] / Ardö leg. [p] 
// ex coll. L. Hromádka / National Museum / Prague, Czech Republic [p] // HOLOTYPUS / PHILONTHUS / batis sp. nov. / Hromádka det., 2015. [p, orange label]'.

Current status. Valid species.

\section{Philonthus bezdeki Hromádka, 2010}

Philonthus bezdeki Hromádka, 2010c: 32, Figs 8-11.

The holotype is deposited in NMPC (ex coll. L. Hromádka):

Holotype $\left(q\right.$, dissected genitalia on a separate plastic card): ${ }^{\circ}[\mathrm{p}$, orange label] // MALAWI cent. / Dedza env. / 16.xii.2001 / J. Bezděk, leg. [p, yellow label] // ex coll. L. Hromádka / National Museum / Prague, Czech Republic [p] // HOLOTYPUS / PHILONTHUS (s. str.) / bezdeki sp. nov. 9 / Hromádka, det. 2005 [p, red label]'.

Current status. Valid species.

\section{Philonthus bitis Hromádka, 2013}

Philonthus bitis Hromádka, 2013e: 209, Figs 13-15.

The holotype is deposited in NMPC (ex coll. L. Hromádka): Holotype (う): ‘Kivu : Terr. Mwenga, / S.-O. Hombwe, Luiko / 2380 m 23-I-1952 [p] // ex coll. L. Hromádka / National Museum / Prague, Czech Republic [p] // HOLOTYPUS / PHILONTHUS (s. str.) / bitis sp. nov. / Hromádka det. 2009. [p, orange label]'.

Current status. Valid species.

\section{Philonthus bleda Hromádka, 2013}

Philonthus bleda Hromádka, 2013e: 209, Figs 16-19.

The holotype is deposited in NMPC (general collection): HoLOTYPE (ふ): 'RWANDA / Nyakabuye / 10.-25.4.1984 / leg.H.Mühle [p] // ex coll. L. Hromádka / National Museum / Prague, Czech Republic [p] // HOLOTYPUS / PHILONTHUS (s. str.) / bleda sp. nov. / Hromádka, det., 2010. [p, orange label]'.

Current status. Valid species.

\section{Philonthus boreas Smetana, 1995}

Philonthus boreas Smetana, 1995: 450, Figs 744-747.

One paratype is deposited in NMPC (ex coll. L. Hromádka):

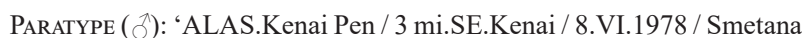
\& Becker [p] // ex coll. L. Hromádka / National Museum / Prague, Czech Republic [p] // PARATYPE / Philonthus / boreas / A. Smetana 1993 / CNC No.21976 [p, yellow label]’.

Current status. Valid species, see Herman (2001b).

\section{Philonthus brendelli Hromádka, 2008}

Philonthus brendelli Hromádka, 2008a: 39, Figs 6-8.

The holotype and one paratype are deposited in NMPC (general collection and ex coll. L. Hromádka):

Holotype (ð): 'Uamgebiet / Bosum 11.-20.6. / 14 Tessmann S. [p, blue label] // ex coll. L. Hromádka / National Museum / Prague, Czech Republic [p] // HOLOTYPUS / PHILONTHUS s. str. / brendelli sp. n. / Hromádka, det. 2006. [p, orange label]'.

Paratype (ठ): 'Quango Gebiet / v. Mechow. [p, violet label] // ex coll. L. Hromádka / National Museum / Prague, Czech Republic [p] // PARATYPUS / PHILONTHUS s. str. / brendelli sp. n. / Hromádka, det. 2006. [p, orange label]'.

Current status. Valid species.
Philonthus breviceps Hromádka, 2013

Philonthus breviceps Hromádka, 2013e: 210, Figs 20-23.

Six paratypes are deposited in NMPC (ex coll. Hromádka):

PARATYPES (2 0 ): 'S.E. Tshamugussa / 2300m 16-VII-1964 / Tam. sous Bambous [p] // Coll. Mus. Tervuren / Zaire: P.N. Virunga / Volcan Sabinyo / R.P. Celis VII-64 [p] // ex coll. L. Hromádka / National Museum / Prague, Czech Republic [p] // PARATYPUS / Philonthus (s. str.) / breviceps sp. nov. / Hromádka det. 2009 [p, orange label]'. Paratype (1 O): 'N. Bitsitsi: au pied / du Sabinyo 2300//2400m / 21VII-1964 [p] // Tamisage / sous / Bambous [p] // Coll. Mus. Tervuren / Zaire: P.N. Virunga / Volcan Sabinyo / R.P. Celis VII-64 [p] // ex coll. L. Hromádka / National Museum / Prague, Czech Republic [p] // PARATYPUS / Philonthus (s. str.) / breviceps sp. nov. / Hromádka det. 2009 [p, orange label]'.

Paratypes (1 J, 1 unsexed specimen): 'Rweru 2900m / 30-VI-1964 / Tamisage [p] // Coll. Mus. Tervuren / Zaire: P.N. Virunga / Volcan Mikeno / R.P. Celis VI-64 [p] // ex coll. L. Hromádka / National Museum / Prague, Czech Republic [p] // PARATYPUS / Philonthus (s. str.) / breviceps sp. nov. / Hromádka det. 2009 [p, orange label]'. Paratype (1 3): 'S.E. de Rweru / 2800m [sic!] 26-VI-1964 [sic!] / Tamisage / Bambousaie [p] // Coll. Mus. Tervuren / Zaire: P.N. Virunga / Volcan Mikeno / R.P. Celis VI-64 [p] // ex coll. L. Hromádka / National Museum / Prague, Czech Republic [p] // PARATYPUS / Philonthus (s. str.) / breviceps sp. nov. / Hromádka det. 2009 [p, orange label]'.

Current status. Valid species.

Philonthus broumandi Boháč, 1981

Philonthus broumandi Boháč, 1981: 357, Figs 5, 6.

One paratype is deposited in NMPC (general collection): PaRATYPE ( $($ ): 'KHOUZESTAN / DEZFOUL : Half-tapeh / 13.5.1975 - 20 m. / PAZOUKI / BROUMAND [p] // Paratypus [p, red label] // Philonthus / broumandi / J. Boháč det. 76 [p]'.

Current status. Valid species, see SchüLKE \& SMETANA (2015).

\section{Philonthus bubalornis Hromádka, 2014}

Philonthus bubalornis Hromádka, 2014d: 688, Figs 1-3.

The holotype and one paratype are deposited in NMPC (general collection and ex coll. L. Hromádka):

HoLotyPe ( đ): 'BOTSWANA(B 11) / Moremi Reservs / $19^{\circ} 23^{\prime} \mathrm{S} 23^{\circ} 33 \mathrm{E}$ [sic!] / 18. - 201972 [p] // ex coll. L. Hromádka / National Museum / Prague, Czech Republic [p] // HOLOTYPUS / PHILONTHUS (s. str.) / bubalornis sp. nov. / Hromádka, det., 2013. [p, red label]'. Paratype ( ${ }^{\prime}$ ): 'BOTSWANA (B 11) / Moremi Reservs / $19^{\circ} 23^{\prime}$ 'S $23^{\circ} 33 \mathrm{E}$ [sic!] / 18. - 201972 [p] // ex coll. L. Hromádka / National Museum / Prague, Czech Republic [p] // PARATYPUS / PHILONTHUS (s. str.) / bubalornis sp. nov. / Hromádka, det., 2013. [p, red label]'.

Current status. Valid species.

\section{Philonthus bubo Hromádka, 2014}

Philonthus bubo Hromádka, 2014j: 416, Figs 1-4.

The holotype is deposited in NMPC (ex coll. L. Hromádka): HoLotype ( $\precsim$ ): 'République Centraafricante [sic!] / Bozo lumiere / 21. V. 1981 / leg. N. Degallier [p] // ex coll. L. Hromádka / National Museum / Prague, Czech Republic [p] // HOLOTY PUS / PHILONTHUS (s. str.) / bubo sp. nov. / Hromádka, det. 2013 [p, red label]’.

Current status. Valid species. 
Philonthus bucorvus Hromádka, 2009

Philonthus bucorvus Hromádka, 2009c: 168, Figs 14-16.

The holotype and five paratypes are deposited in NMPC (general collection and ex coll. L. Hromádka):

Holotype (ð): ‘MALAWI S / Mulanoe Mts. env. / 22. xii. 2001 J. Bezděk, lgt. [p, yellow label] // ex coll. L. Hromádka / National Museum / Prague, Czech Republic [p] // HOLOTYPUS / PHILONTHUS (Philonthus) / bucorvus sp. nov. / Hromádka, det. 2007 [p, orange label]'.

Paratypes (1 $\delta, 3$ unsexed specimens): 'MALAWI S / Mulanoe Mts. env. / 22. xii. 2001 J. Bezděk, lgt. [p, yellow label] // ex coll. L. Hromádka / National Museum / Prague, Czech Republic [p] // PARATYPUS / PHILONTHUS (Philonthus) / bucorvus sp. nov. / Hromádka, det. 2007 [p, orange label]'.

PARATyPe (ふ): 'MALAWI S / Mulanoe Mts. env. / 22. xii. 2001 J. Bezděk, lgt. [p, yellow label] // ex coll. L. Hromádka / National Museum / Prague, Czech Republic [p] // PARATYPUS / PHILONTHUS (Philonthus) / bucorvus sp. nov. / Hromádka, det. 2008 [p, red label]'.

Current status. Valid species.

Comments. HROMÁDKA (2009c) stated in the original description, that all paratypes are females. However, two paratypes are in fact dissected males; we have no doubts about their authenticity.

Philonthus busiris Smetana, 1995

Philonthus busiris Smetana, 1995: 358, Figs 555-560.

One paratype is deposited in NMPC (ex coll. L. Hromádka): Paratype (unsexed specimen): 'ONT. Carleton Co. / Fitzroy Prov. Pk. / 2-3.V.1979 / A\&Z Smetana [p] // ex coll. L. Hromádka / National Museum / Prague, Czech Republic [p] // PARATYPE / Philonthus / busiris / A. Smetana 1993 / CNC No.21965 [p, yellow label]'.

Current status. Valid species, see Herman (2001b).

Philonthus calabaria Hromádka, 2012

Philonthus calabaria Hromádka, 2012c: 140, Figs 11-13.

The holotype is deposited in NMPC (general collection):

Holotype (ठ): ‘ $\lesssim$ [p, red label] // ETHIOPIA - Bale / Sabsebe Washa N Park / $07^{\circ} 03^{\prime}$ N $39^{\circ} 39 E$ Esic!] / 3.600 m 1971 [p] // ex coll. L. Hromádka / National Museum / Prague, Czech Republic [p] // HOLOTYPUS / PHILONTHUS (s. str.) / calabaria sp.nov. / Hromádka, det. 2010. [p, orange label]'.

Current status. Valid species.

\section{Philonthus camelus Hromádka, 2008}

Philonthus (s. str.) camelus Hromádka, 2008c: 214, Figs 14-19.

The holotype is deposited in NMPC (ex coll. L. Hromádka): Holotype ( $\left.\jmath^{\jmath}\right)$ : ‘ $\left[\mathrm{p}\right.$, red label] // VR Congo, $4,30^{\circ} / \mathrm{S} / 13,96^{\circ} \mathrm{O}, 210 \mathrm{~m}$ / Bouansa,3.5.85 / lux,leg.Bremer [p] // ex coll. L. Hromádka / National Museum / Prague, Czech Republic [p] // HOLOTYPUS PHILONTHUS (s. str.) / camelus sp. n. §̧ / L. Hromádka, det. 2006 [p, red label]'.

Current status. Valid species.

Comments. According to the original description, HromáDKa (2008c) described $P$. camelus based on the holotype from 'TANZANIA ('Allemande') Afrique or., Kilimandjaro, versant sud-est, Alluaud-Jeannel'. However, the holotype specimen in Hromádka's collection has the locality label from Congo (see above). As the voucher specimen agrees well with the original description and especially with the drawings of male genitalia, we consider the specimen to be the real holotype and assume that Hromádka probably used some old description as a template for $P$. camelus but forgot to change the type material section. Consequently, the type locality and the distribution of $P$. camelus is corrected from Tanzania: Kilimandjaro to Congo: Bouansa.

\section{Philonthus canescens Mäklin, 1852}

Philonthus canescens Mäklin, 1852: 313

Two paralectotypes are deposited in NMPC (general collection):

PARALECTOTYPes (2 unsexed specimens): 'Kadjak [p] // Holmberg [p] // Spec. typ.No. [p] / Phil. canescens / Mākl. Cotype [hw, red label] // PARALECTOTYPE / PHILONTHUS / canescens Mäklin, 1852 / labelled by NMPC, 2019 [p, red label]'.

Current status. Cafius (Bryonomus) canescens (Mäklin, 1852), see e.g. DVOŘÁK (1957) and HerMan (2001b).

Comments. MäKLIN (1852) described this species from an unknown number of specimens. The lectotype was designated by ORTH \& MOORE (1980). Because there is no doubt about the authenticity of our specimens, we have labeled them as additional paralectotypes.

\section{Philonthus canis Hromádka, 2012}

Philonthus canis Hromádka, 2012c: 140, Figs 14-16.

Two paratypes are deposited in NMPC (ex coll. L. Hromádka):

ParatyPes ( $1 \delta 1$ ) $)$ : 'KENYA-Western / Kakamega Forest N.R. / prim. Forest, $1600 \mathrm{~m} / 0.21,34 \mathrm{~N} / 34.51,39 \mathrm{E} /$ 18.ix.2001, light trap 1(2) / leg. L.Kühne \& J.Holstein [p, blue label] // ex coll. L. Hromádka / National Museum / Prague, Czech Republic [p] // PARATYPUS / PHILONTHUS (s. str.) / canis sp.n. / Hromádka, det. 2006. [p, orange label]'.

Current status. Valid species.

Philonthus centropus Hromádka, 2014

Philonthus centropus Hromádka, 2014j: 417, Figs 10-13.

The holotype is deposited in NMPC (general collection): Holotype ( $\left.\mathcal{O}^{\circledR}\right)$ : 'Süd Afrika / Kapland [p] // ex coll. L. Hromádka / National Museum / Prague, Czech Republic [p] // HOLOTYPUS / PHILONTHUS (s. str.) / centropus sp. nov. / Hromádka, det. 2013 [p, red label]'.

Current status. Valid species.

\section{Philonthus centropyge Hromádka, 2012}

Philonthus centropyge Hromádka, 2012c: 142, Figs 17-19.

The holotype is deposited in NMPC (general collection):

HoLOTYPE (ð): 'TANZANIA / Uluguru Mts. / Mwanihana Forest / above Sanje / 1700m [p] // ex coll. L. Hromádka / National Museum / Prague, Czech Republic [p] // HOLOTYPUS / PHILONTHUS (s. str.) / pelomedusa sp.nov. / Hromádka, det., 2011. [p, red label]'.

Current status. Philonthus pelomedusa Hromádka, 2013. Comments. HromádKa (2013f) proposed the new substitute name Philonthus pelomedusa Hromádka, 2013 for Philonthus centropyge Hromádka, 2012, a junior primary homonym of Philonthus centropyge Hromádka, 2011. 
Philonthus cephalopholis Hromádka, 2011

Philonthus cephalopholis Hromádka, 2011b: 46, Figs 23-27.

The holotype is deposited in NMPC (general collection):

Holotype (ð): ‘Kivu:Mulungu-Tshibinda / XI. 1951 / P. C. Lefèvre [p] // ex coll. L. Hromádka / National Museum / Prague, Czech Republic [p] // HOLOTYPUS / PHILONTHUS (s. str.) / cephalopholis sp. nov. / Hromádka, det. 2009. [p, orange label]'.

Current status. Valid species.

\section{Philonthus cerambus Smetana, 1995}

Philonthus cerambus Smetana, 1995: 101, Figs 64-70.

One paratype is deposited in NMPC (ex coll. L. Hromádka):

PARATYPE (unsexed specimen): 'N.M.Lincoln N.F.lmi. / SE.Cloudcroft 8750، / 13-18.VII.1969 / A.Smetana [p] // ex coll. L. Hromádka / National Museum / Prague, Czech Republic [p] // PARATYPE / Philonthus / cerambus / A. Smetana 1993 / CNC No.21948 [p, yellow label]'.

Current status. Valid species, see Herman (2001b).

\section{Philonthus ceryle Hromádka, 2013}

Philonthus ceryle Hromádka, 2013d: 389, Figs 24-27.

The holotype is deposited in NMPC (general collection): Holotype (§): 'CAMEROON / Mhalmayo F.Res. / TIGER Survey, [p] // ex coll. L. Hromádka / National Museum / Prague, Czech Republic [p] // HOLOTYPUS / PHILONTHUS (s. str.) / ceryle sp. nov. / Hromádka, det. 2009 [p, orange label]'.

Current status. Valid species.

\section{Philonthus civettictis Hromádka, 2011}

Philonthus civettictis Hromádka, 2011b: 48, Figs 28-31.

Three paratypes are deposited in NMPC (ex coll. L. Hromádka):

Paratypes $(2 \lesssim \hat{\delta})$ : 'COLL. MUS. CONGO / Lulua : Kapanga / III1933/ F. G. Overlaet [p] // ex coll. L. Hromádka / National Museum / Prague, Czech Republic [p] // PARATY PUS / PHILONTHUS (s. str.) / civettictis sp. nov. / Hromádka, det., 2010. [p, orange label]'.

Paratype (1 đ): 'ANGOLA (A 25) / Rio Longs / 4 mls S. Lussusso / 8. iii. 1972 [p] // ex coll. L. Hromádka / National Museum / Prague, Czech Republic [p] // PARATYPUS / PHILONTHUS (s. str.) / civettictis sp. nov. / Hromádka, det., 2010. [p, orange label]'.

Current status. Valid species.

Philonthus clanga Hromádka, 2015

Philonthus clanga Hromádka, 2015a: 60, Fig. 1.

One paratype is deposited in NMPC (ex coll. L. Hromádka):

PARATYPe (unsexed specimen): 'TANZANIA 1807m / Mt. Longido / S02 42'39";E36 43 '31" / 6-9.viii.12 in buffalo dung / leg. Smith, Takano \& Garner [p] // BMNH $\{\mathrm{E}\} /$ 2012-92 [p] // BMNH(E) / 1305123 [p] // ex coll. L. Hromádka / National Museum / Prague, Czech Republic [p] // PARATY PUS / PHILONTHUS (s. str.) / clanga sp.nov. / Hromádka, det., 2014. [p, red label]'.

Current status. Valid species.

\section{Philonthus coiffaiti Hromádka, 1992}

Philonthus [s.str.] coiffaiti Hromádka, 1992: 97, Figs 1-2.

The holotype is deposited in NMPC (ex coll. L. Hromádka): HoLotype ( ROUSEK [hw] // ex coll. L. Hromádka / National Museum / Prague,
Czech Republic [p] // HOLOTYPUS / PHILONTHUS (s. str.) / coiffaiti sp. nov. / Hromádka, det., 1992. [p, orange label] // Philonthus / purpuripennis REITTER / det. Schillhammer 1999 [p]'.

Current status. Junior subjective synonym of Philonthus purpuripennis Reitter, 1887, and junior homonym of Philonthus coiffaiti Levasseur, 1962, see SCHILLHAMmER (1999) and SCHÜLKE \& SMETANA (2015).

Philonthus colius Hromádka, 2008

Philonthus colius Hromádka, 2008b: 52, Figs 1-6.

One paratype is deposited in NMPC (ex coll. L. Hromádka): Paratype ( (ð): 'ZAMBIA NW / 27 km N Kasempa / 10.12.2004 / Snížek \& Tichý [p] // ex coll. L. Hromádka / National Museum / Prague, Czech Republic [p] // PARATY PUS / PHILONTHUS(Raucalius) / colius sp. nov. / Hromádka, det. 2007 [p, orange label]'.

Current status. Valid species.

Philonthus colius Hromádka, 2016

Philonthus colius Hromádka, 2016c: 374, Figs 7-9.

Two paratypes are deposited in NMPC (ex coll. L. Hromádka):

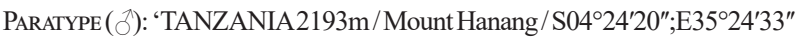
/31.vii-4.viii.12 Dung Pitfall / leg. Smith, Takano \& Garner [p] // BMNH \{E\} / 2012-92 [p] // BMNH(E) / 1262485 [p] // ex coll. L. Hromádka / National Museum / Prague, Czech Republic [p] // PARATYPUS / PHILONTHUS (s. str.) / colius sp. nov. / Hromádka, det., 2015 [p, red label]'.

PARATYPE (unsexed specimen): 'TANZANIA 2193m / Mount Hanang / S04²4'20"; E35 24'33" / 31.vii-4.viii.12 Dung Pitfall / leg. Smith, Takano \& Garner [p] // BMNH $\{\mathrm{E}\} /$ 2012-92 [p] // BMNH(E) / 1262507 [p] // ex coll. L. Hromádka / National Museum / Prague, Czech Republic [p] // PARATY PUS / PHILONTHUS (s. str.) / colius sp. nov. / Hromádka, det., 2015 [p, red label]'.

Current status. Philonthus lubomirhromadkai Geiser \& Barclay, 2017.

Comments. GEISER \& BARCLAY (2017) proposed the new substitute name Philonthus lubomirhromadkai Geiser \& Barclay, 2017 for Philonthus colius Hromádka, 2016, a junior primary homonym of Philonthus colius Hromádka, 2008.

Philonthus colobus Hromádka, 2012

Philonthus colobus Hromádka, 2012c: 145, Figs 24-26.

The holotype is deposited in NMPC (general collection): Holotype (§): 'Rwanda: Prov. / Cyangugu: / Nyakabuye [p] // 16.XII.1985/ leg.H.Mühle [p] // ex coll. L. Hromádka / National Museum / Prague, Czech Republic [p] // HOLOTY PE / PHILONTHUS (s. str.) / colobus sp.nov. / Hromádka, det. 2011. [p, orange label]'

Current status. Valid species.

\section{Philonthus coris Hromádka, 2011}

Philonthus coris Hromádka, 2011b: 50, Figs 37-41.

The holotype is deposited in NMPC (general collection): HoLOTYPe (ð): 'CONGO BELGE / Edouard: Vitshumbi / U.V. 7.V. 1954 / J. Verbecke - KEA [p] // ex coll. L. Hromádka / National Museum / Prague, Czech Republic [p] // HOLOTY PUS / PHILONTHUS (s. str.) / coris sp. nov. / Hromádka, det. 2009. [p, orange label]'.

Current status. Valid species. 
Philonthus crecopsis Hromádka, 2013

Philonthus crecopsis Hromádka, 2013e: 212, Figs 27-29.

The holotype is deposited in NMPC (general collection):

Holotype (§): 'CONGO BELGE / Edouard: Vitshumbi / U.V. 7.V. 1954 [sic!] / J. Verbecke - KEA [p] // ex coll. L. Hromádka / National Museum / Prague, Czech Republic [p] // HOLOTYPUS / PHILONTHUS (s. str.) / crecopsis sp. nov. / Hromádka, det. 2013. [p, red label]'.

Current status. Valid species.

Comments. HromáDKa (2013e: 203, 212, 214) used two different original spellings, Philonthus creopsis and $P$. crecopsis in the publication. As the specific epithet is derived from the Latin generic name of the African crake Crecopsis egregia (Peters, 1854), we fix the correct original spelling of the species Philonthus crecopsis according to Article 32.2.1 (ICZN 1999).

\section{Philonthus cricetomys Hromádka, 2014}

Philonthus cricetomys Hromádka, 2014j: 419, Figs 27-29.

One paratype is deposited in NMPC (ex coll. L. Hromádka):

PARATYPe (ठ): 'TANZANIA 2039m / Igoma, Chome NR / South Pare Mountains / S04 17'42";E37 56'17" / 5-7.xii.11 Dung Pitfall / leg. Smith,R \& Takano,H [p] // BMNH \{E\} / 2011-88 [p] // BMNH\# / 1026882 [p] // ex coll. L. Hromádka / National Museum / Prague, Czech Republic [p] // PARATYPUS / PHILONTHUS (s. str.) / cricetomys sp. nov. / Hromádka, det., 2012. [p, orange label]'.

Current status. Philonthus botaurus Hromádka, 2016. Comments. HromádKa (2016a) proposed the new substitute name Philonthus botaurus Hromádka, 2016 for Philonthus cricetomys Hromádka, 2014, a junior primary homonym of Philonthus cricetomys Paulian, 1952.

Philonthus crocidura Hromádka, 2011

Philonthus crocidura Hromádka, 2011c: 161, Figs 16-18.

One paratype is deposited in NMPC (ex coll. L. Hromádka):

ParatyPe (ठ): ‘I.R.S.A.C.-MUS.CONGO / Kivu : Terr. Kalehe, / Contr. S. O. Kahuzi, / 2200 m. VIII-51 / N. Leleup [sic!] [p] // Récolté humus / Bambous / et Hagenia [p, green label] // ex coll. L. Hromádka / National Museum / Prague, Czech Republic [p] // PARATYPUS / PHILONTHUS (s. str.) / crocidura sp. nov. / Hromádka, det., 2008. [p, red label]'.

Current status. Valid species.

Philonthus crocodylus Hromádka, 2010

Philonthus crocodylus Hromádka, 2010e: 20, Figs 56-60.

One paratype is deposited in NMPC (ex coll. L. Hromádka): PARATyPe ( + ): '722 [p, yellow label] // Ebogo. / (?).vii.1993 / FITrap 1. [p] // CAMEROON: / Mbalmayo F.Res. / TIGER Survey. / BM 1994-148. [p] // ex coll. L. Hromádka / National Museum / Prague, Czech Republic [p] // PARATY PUS / PHILONTHUS (Pseudophil.) / crocodylus sp. nov. / Hromádka det. 2008 [p, pink label]'.

Current status. Valid species.

\section{Philonthus csikii Bernhauer, 1917}

Philonthus Csikii Bernhauer, 1917: 46.

One syntype is deposited in NMPC (general collection):

SyNTYPE (unsexed specimen): ‘Africa or. / Katona [p] // Arusha-Ju / 1905. XII. [sic!] [p] // Csikii Brh / cotypus [hw]'.

Current status. Valid species, see Herman (2001b).
Philonthus cuculus Hromádka, 2013

Philonthus cuculus Hromádka, 2013b: 4, Figs 8-10.

The holotype is deposited in NMPC (general collection): HоLотуре (ठ): 'SIERRA LEONE / Western Area / Base Picket Hill / 9.i.1997 W. Rossi [p, green label] // ex coll. L. Hromádka / National Museum / Prague, Czech Republic [p] // HOLOTYPUS / PHILONTHUS (s. str.) / cuculus sp. nov. / Hromádka det., 2012. [p, red label]'.

Current status. Valid species.

Philonthus dasypeltis Hromádka, 2010

Philonthus dasypeltis Hromádka, 2010e: 22, Figs 61-63.

Two paratypes are deposited in NMPC (ex coll. L. Hromádka):

Paratypes ( 1 ภ, 1 unsexed specimen): 'Angola: Parc Dr. / Carrisso, nr. / Dundo / 6, XI. 1972 [p] // ex debris / piles / Nest No. 1 [p] 9 [hw] 5 [p] // Coll. D.H.Kistner / \& R.J. Swift / No. 2 [p] 548 [hw] // ex coll. L. Hromádka / National Museum / Prague, Czech Republic [p] // PARATY PUS / PHILONTHUS (Philont.) / dasypeltis sp. nov. / Hromádka, det. 2006 [p, orange label]'.

Current status. Valid species.

Philonthus davus Smetana, 1995

Philonthus davus Smetana, 1995: 97, Figs 49-55.

Two paratypes are deposited in NMPC (ex coll. L. Hromádka):

Paratypes (2 unsexed specimens): 'CAL. S.Bern.Mts. / Hwy.38 3miSW Onyx / Summit 7700’ 14. / III.83 A.Smetana [p] // ex coll. L. Hromádka / National Museum / Prague, Czech Republic [p] // PARATYPE / Philonthus / davus / A. Smetana 1993 / CNC No.21961 [p, yellow label]'.

Current status. Valid species, see Herman (2001b).

Philonthus dendroaspis Hromádka, 2008

Philonthus dendroaspis Hromádka, 2008b: 53, Figs 7-12.

The holotype is deposited in NMPC (general collection): Holotype (ð): 'ZAMBIA C / 140 km NE of Kapiri / Mposhi [sic!], 40km SW / Serenje, 30.11.2004 / Snížek \& Tichý lgt. [p] // ex coll. L. Hromádka / National Museum / Prague, Czech Republic [p] // HOLOTYPUS / PHILONTHUS (Raucalius) / dendroaspis sp. nov. / Hromádka, det., 2007 [p, orange label]'.

Current status. Valid species.

Philonthus dendropicos Hromádka, 2012

Philonthus dendropicos Hromádka, 2012e: 178, Figs 32-35.

The holotype is deposited in NMPC (ex coll. L. Hromádka): HoLOTYPe $\left({ }^{\jmath}\right)$ : 'ZIMBABWE / loc. Kutsaga / near Harare airport / 18.vi.1997 W. Rossi leg. [p, yellow label] // ex coll. L. Hromádka / National Museum / Prague, Czech Republic [p] // HOLOTYPUS / PHILONTHUS (s. str.) / dendropicos sp. nov. / Hromádka, det., 2012. [p, orange label]',

Current status. Valid species.

Philonthus diceros Hromádka, 2011

Philonthus diceros Hromádka, 2011b: 53, Figs 62-65.

Four paratypes are deposited in NMPC (ex coll. L. Hromádka): 
ParatyPes (1 $\delta$, 1 unsexed specimen): 'KENYA-Western / Kakamega Forest N.R. / env. Udo's Campsite / 0.21N / 34.51 E / 1600m, 27.-29. ix.2001 / light trap, leg.L.Kühne [p, blue label] // ex coll. L. Hromádka / National Museum / Prague, Czech Republic [p] // PARATYPUS / PHILONTHUS (s. str.) / diceros sp. n. / Hromádka, det. 2006. [p, orange label]'.

PARATYPe (ð): 'KENYA-Western / Kakamega Forest N.R. / env. Udo's Campsite / 0.21N / 34.51 E / 1600m, 27.-29.ix.2001 / light trap, leg.L.Kühne [p, blue label] // ex coll. L. Hromádka / National Museum / Prague, Czech Republic [p] // PARAT Y PUS / PHILONTHUS (s. str.) / diceros sp. n. đ/ Hromádka, det. 2006. [p, orange label]'.

PARATyPe (unsexed specimen): 'KENYA-Western / Kakamega Forest N.R. / prim. Forest, 1600m/0.21N/34.51 E/ 18.ix.2001,lighttrap 1(2)/leg. L. Kühne \& J.Holstein [p, blue label] // ex coll. L. Hromádka / National Museum / Prague, Czech Republic [p] // PARATYPUS / PHILONTHUS (s. str.) / diceros sp. n. / Hromádka, det. 2006. [p, orange label]'.

Current status. Valid species.

Philonthus dinemellia Hromádka, 2014

Philonthus dinemellia Hromádka, 2014j: 418, Figs 21-23.

The holotype is deposited in NMPC (general collection): HоLотуре (ठ): ‘30-3-1983 / Lama Kara [sic!] / Togo;leg.Krell [p] // Hotel Kara / Hg-lux [p] // ex coll. L. Hromádka / National Museum / Prague, Czech Republic [p] // HOLOTYPUS / PHILONTHUS (s. str.) / dinemellia sp. nov. / Hromádka, det. 2012. [p, orange label]'.

Current status. Valid species.

\section{Philonthus emberiza Hromádka, 2011}

Philonthus emberiza Hromádka, 2011a: 192, Figs 9-14.

One paratype is deposited in NMPC (ex coll. L. Hromádka):

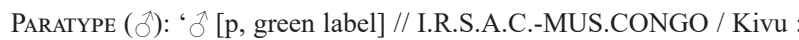
Terr. Kabare, / Nyakasiba, 1800 m. [p] / (vestige forêt) [hw] VI-1951 / N. Leleup [p] // ex coll. L. Hromádka / National Museum / Prague, Czech Republic [p] // PARATYPUS / PHILONTHUS (s. str.) / emberiza sp. nov. / Hromádka, det. 2009. [p, red label]'.

Current status. Valid species.

\section{Philonthus epomops Hromádka, 2012}

Philonthus epomops Hromádka, 2012c: 149, Figs 33-35.

The holotype is deposited in NMPC (ex coll. L. Hromádka):

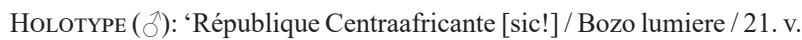
1981 / leg. N. Degallier [p] // ex coll. L. Hromádka / National Museum / Prague, Czech Republic [p] // HOLOTYPUS / PHILONTHUS (s. str.) / epomops sp.nov. / Hromádka, det. 2011. [p, orange label]'

Current status. Valid species.

Philonthus eremitalpa Hromádka, 2013

Philonthus eremitalpa Hromádka, 2013g: 703, Figs 1-2.

The holotype and two paratypes are deposited in NMPC (general collection and ex coll. L. Hromádka):

HoLotype (ð): ‘République Centraafricante [sic!] / Bozo lumiere / 21.v. 1981. / leg. N. Degallier [p] // ex coll. L. Hromádka / National Museum / Prague, Czech Republic [p] // HOLOTY PUS / PHILONTHUS (s. str.) / eremitalpa sp. nov. / Hromádka, det. 2012. [p, orange label]'.

Paratypes (2 unsexed specimens): 'République Centraafricante [sic!] / Bozo lumiere / 21. v. 1981. / leg. N. Degallier [p] // ex coll. L. Hromádka / National Museum / Prague, Czech Republic [p] // PARATYPUS / PHILONTHUS (s. str.) / eremitalpa sp. nov. / Hromádka, det. 2012. [p, orange label]'.

Current status. Valid species.
Philonthus estrilda Hromádka, 2010

Philonthus estrilda Hromádka, 2010f: 57, Figs 7-8

The holotype is deposited in NMPC (general collection): HoLotype (đ): ‘ ‘ [p, pink label] // Lulua : Kapanga [sic!] / IV-1933 / F. G. Overlaet [p] // ex coll. L. Hromádka / National Museum / Prague, Czech Republic [p] // HOLOTYPUS / PHILONTHUS (s. str.) / estrilda sp. nov. / Hromádka, 2010. [p, orange label]'.

Current status. Valid species.

Philonthus euplectes Hromádka, 2013

Philonthus euplectes Hromádka, 2013f: 714, Figs 1-5.

The holotype is deposited in NMPC (ex coll. L. Hromádka): HoLotype (ð̋): ‘TANZANIA / Mwanza / 11.x.1969 / Ardö leg. [p] // ex coll. L. Hromádka / National Museum / Prague, Czech Republic [p] // HOLOTYPUS / PHILONTHUS (s. str.) / euplectes sp. nov. / Hromádka, det., 2012. [p, red label]'.

Current status. Valid species.

Philonthus falco Hromádka, 2009

Philonthus falco Hromádka, 2009c: 174, Figs 30-34.

Five paratypes are deposited in NMPC (ex coll. L. Hromádka):

PARATYPE ( 0 , dissected genitalia on a separate plastic card): 'ZIMBA-

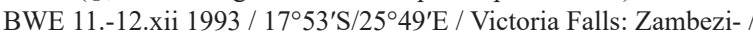
NP-Camp,lux,leg.M.Uhlig [p] // ex coll. L. Hromádka / National Museum / Prague, Czech Republic [p] // PARATYPUS / PHILONTHUS (Philonthus) / falco sp. nov. / Hromádka, det., 2007. [p, orange label]'.

ParatyPes (1 1 , 1 unsexed specimen): 'SIMBABWE: Save-Fluss / ca. $100 \mathrm{~km} \mathrm{~S}$ Mutare, $770 \mathrm{~m} / 19^{\circ} 53^{\prime} \mathrm{S} / 32^{\circ} 22^{\prime} \mathrm{E} / 8$. III.2000, leg.U.Heinig / Lichtfang [p, blue label] // Sammlung / M.Schülke / Berlin [p] // ex coll. L. Hromádka / National Museum / Prague, Czech Republic [p] // PAR ATYPUS / PHILONTHUS (Philonthus) / falco sp. nov. / Hromádka, det., 2007. [p, orange label]'.

ParatyPe (unsexed specimen): 'Madagascar 10.-27.XII.2003 / 20 km N Tulear, / ifaty, $30 \mathrm{~m}$ [sic!] / S.Murzin\&A.Shamaev leg. [p] // Philonthus / sp. 5 (caffer gr.) / J. Janák det. 2007 [p] // ex coll. L. Hromádka / National Museum / Prague, Czech Republic [p] // PARATY PUS / PHILONTHUS (Philonthus) / falco sp. nov. / Hromádka, det., 2007. [p, orange label]'.

PARATYPe (unsexed specimen): 'SW Madagascar 2002 / Morondava distr. / Kirindy Forest / D. Hauck lgt. 5.-8.1. [p] // Philonthus / sp. 5 (caffer gr.) / J. Janák det. 2007 [p] // ex coll. L. Hromádka / National Museum / Prague, Czech Republic [p] // PARATYPUS / PHILONTHUS (Philonthus) / falco sp. nov. / Hromádka, det., 2007. [p, orange label]'.

Current status. Valid species.

\section{Philonthus femoralis Mäklin, 1853}

Philonthus femoralis Mäklin, 1853: 189.

Two paralectotypes are deposited in NMPC:

Paralectotypes (2 unsexed specimens): 'Kadjak [p] // Holmberg [p] // Spec. typ.No. [p] / Phil. femoralis / Mākl. Cotype [hw, red label] // PARALECTOTYPE / PHILONTHUS / femoralis Mäklin, 1853 / labelled by NMPC, 2019 [p, red label]’.

Current status. Cafius mutatus (Gemminger \& Harold, 1868), see Herman (2001b).

Comments. Gemminger \& Harold (1868) proposed the new substitute name Philonthus mutatus Gemminger \& Harold, 1868 for Philonthus femoralis Mäklin, 1853, a junior primary homonym of Philonthus femoralis Hochhuth, 1851 (currently in the genus Gabrius Stephens, 1829). 
MäKLIN (1853) described P. femoralis from an unknown number of specimens. The lectotype was designated by ORTH \& MOORE (1980). Because there is no doubt about the authenticity of our specimens, we have labeled them as additional paralectotypes.

Philonthus ferreipennis stigmatias Smetana, 1995

Philonthus ferreipennis stigmatias Smetana, 1995: 350.

One paratype is deposited in NMPC (ex coll. L. Hromádka):

PARATyPe (unsexed specimen): 'CAL.S.Diego Co. / Laguna Mts. Little / Laguna Lk.5600 5. / III.83 A.Smetana [p] // ex coll. L. Hromádka / National Museum / Prague, Czech Republic [p] // PARATYPE / Philonthus / ferreipennis / stigmatias / A. Smetana 1993 / CNC No.21963 [p, yellow label]'.

Current status. Valid subspecies, see Herman (2001b).

Philonthus fungicelebrans Janák \& Lecoq, 2005

Philonthus fungicelebrans Janák \& Lecoq, 2005: 289, Figs 1-9.

One paratype is deposited in NMPC (ex coll. L. Hromádka):

PARATyPe (đ): 'E Madagascar 24. 12. 1998 / 30km ESE of Betroka, 1600/ 1650m, Vohitrosa forest,3km / NE of $\boldsymbol{\Delta} 1825 \mathrm{~m}$, J. Janák lgt. [p] // rain forest / in mushrooms / on fallen tree [p] // ex coll. L. Hromádka / National Museum / Prague, Czech Republic [p] // PARATYPUS $\delta$ / PHILONTHUS sp. n. / fungicelebrans / Janák \& Lecoq det. 2004 [hw, red label]'.

Current status. Valid species.

\section{Philonthus galago Hromádka, 2010}

Philonthus galago Hromádka, 2010a: 137, Figs 15-24.

The holotype and two paratypes are deposited in NMPC (general collection and ex coll. Hromádka):

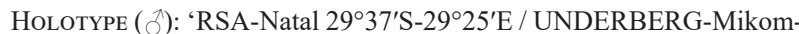
azana / Lodge 1400-1800 m / Dr.R.Fencl lgt. 11.11.2001 [sic!] [p] // ex coll. L. Hromádka / National Museum / Prague, Czech Republic [p] // HOLOTY PUS / PHILONTHUS (Philonthus) / galago sp. nov. / Hromádka, det. 2007 [p, orange label]'.

Paratype ( + ): 'I.R.S.A.C.-MUS. CONGO / Kundelungu 1750 m [p] / 6-III- [hw] 1950 / N. Leleup [p] / (Ilôt boisé dans tourbière) [hw] // ex coll. L. Hromádka / National Museum / Prague, Czech Republic [p] // PARATY PUS / PHILONTHUS (Philon.) / galago spec. nov. / Hromádka, det. 2007 [p, orange label]'.

Paratype (unsexed specimen): 'Zambie [p] VII.1985 / Lusaka [hw] / H. Burda lgt. [p] // ex coll. L. Hromádka / National Museum / Prague, Czech Republic [p] // PARATY PUS / PHILONTHUS (s. str.) / galago sp. nov. / Hromádka, det. 2007 [p, orange label]'.

Current status. Valid species.

Philonthus giraffa Hromádka, 2010

Philonthus giraffa Hromádka, 2010e: 25, Figs 68-72.

The holotype is deposited in NMPC (general collection):

HoLOTYPe ( $\partial$, dissected genitalia on a separate plastic card): 'SIERRA LEONE / N.P. Kabala / alla luce [p] // ex coll. L. Hromádka / National Museum / Prague, Czech Republic [p] // HOLOTYPUS / PHILONTHUS (Pseudoph.) / giraffa sp. nov. / Hromádka, det. 2006 [p, red label]'.

Current status. Valid species.

Philonthus graphiurus Hromádka, 2011

Philonthus graphiurus Hromádka, 2011g: 1347, Figs 5-8.

The holotype is deposited in NMPC (general collection):
Holotype ( 3 ): 'République / Centrafricaine / BOZO, lumiére / 21.5.1981 / leg. N. Degallier [p] // ex coll. L. Hromádka / National Museum / Prague, Czech Republic [p] // HOLOTYPUS / PHILONTHUS (s. str.) / graphiurus sp. nov. / Hromádka, det. 2011 [p, red label]'.

Current status. Valid species.

Philonthus gypaetus Hromádka, 2010

Philonthus gypaetus Hromádka, 2010e: 26, Figs 73-76.

The holotype is deposited in NMPC (general collection): HoLOTYPe (đ): 'Zambie / 7. 1985 / Lusaka / M. Burda leg. [p, yellow label] // ex coll. L. Hromádka / National Museum / Prague, Czech Republic [p] // HOLOTYPUS / PHILONTHUS (Pseudoph.) / gypaetus sp. nov. / Hromádka, det. 2008 [p, red label]’.

Current status. Valid species.

Philonthus haliaeetus Hromádka, 2010

Philonthus haliaeetus Hromádka, 2010e: 28, Figs 77-80.

The holotype and one paratype are deposited in NMPC (general collection and ex coll. Hromádka):

HoLOTYPe (ð): 'NIGERIA / In Abangori / 3.ii.1978 / R.Macek, lgt [p] // ex coll. L. Hromádka / National Museum / Prague, Czech Republic [p] // HOLOTYPUS / PHILONTHUS / manis / Hromádka, det., 2008. [p, orange label] // HOLOTYPE // PHILONTHUS / haliaeetus Hromádka, 2010 / labelled by NMPC, 2019 [p, red label]'.

ParatyPe ( $₫$ [sic!]): 'CONGO / Lulua Kapanga / X.1932 / G.F. Overlaet lgt. [p] // ex coll. L. Hromádka / National Museum / Prague, Czech Republic [p] // PARATYPUS / PHILONTHUS / manis / Hromádka, det., 2008. [p, orange label] // PARATYPE // PHILONTHUS / haliaeetus Hromádka, 2010 / labelled by NMPC, 2019 [p, red label]'.

Current status. Valid species.

Comments. HromáDKa (2010e, 2011a) described two different species under the name Philonthus haliaeetus. When he became aware of that, he proposed the new substitute name $P$. manis for the junior homonym (see below). Unfortunately, he changed the type labels under both homonymous species.

\section{Philonthus haliaeetus Hromádka, 2011}

Philonthus haliaeetus Hromádka, 2011a: 194, Figs 15-18.

The holotype and one paratype are deposited in NMPC (ex coll. L. Hromádka):

Holotype (ð): 'RWANDA / Lake KIWU / 3-80,Rataj [p] // ex coll. L. Hromádka / National Museum / Prague, Czech Republic [p] // HOLOTYPUS / PHILONTHUS (s. str.) / manis sp. nov / Hromádka, det., 2011. [p, red label]'.

ParatyPe ( $\left.{ }^{1}\right)$ : 'Tanzania / Mwanza .7 / 69 P. Ardö [p] / [reverse of the same label] 4/7 [hw] // ex coll. L. Hromádka / National Museum / Prague, Czech Republic [p] // PARATY PUS / PHILONTHUS (s. str.) / manis sp. nov / Hromádka, det., 2011. [p, red label]'.

Current status. Philonthus manis Hromádka, 2013.

Comments. HromádKa (2013f) proposed the new substitute name Philonthus manis Hromádka, 2013 for Philonthus haliaeetus Hromádka, 2011, a junior primary homonym of Philonthus haliaeetus Hromádka, 2010.

Philonthus haliophis Hromádka, 2011

Philonthus haliophis Hromádka, 2011a: 195, Figs 19-21.

The holotype is deposited in NMPC (general collection):

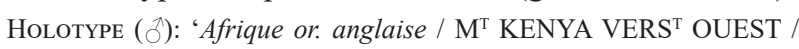


ZONE DES FORÊTS / ALLUAUD \& JEANNEL [p] // FORETS [sic!] MOYENNES / BAMBOUS SUPÊR / 2800-3200m / Janv. 1912 St. 41 [p] // ex coll. L. Hromádka / National Museum / Prague, Czech Republic [p] // HOLOTYPUS / PHILONTHUS (s. str.) / haliophis sp. nov. / Hromádka det. 2009. [p, orange label]'.

Current status. Valid species.

Philonthus hapalemur Hromádka \& Janák, 2011

Philonthus (Philonthus) hapalemur Hromádka \& Janák, 2011: 138, Figs 1-9.

One paratype is deposited in NMPC (ex coll. L. Hromádka):

PARAType (ठ): 'MADAGASCAR 1075m [p] // Antsiranana, Antsahampano / Montagne d'Ambre / Malaise Trap, 15-19.xii. 2004 / $12.53^{\circ} \mathrm{S}$ 49.17E, D.C.Lees / BMNH \{E\} 2004-46 [p] // ex coll. L. Hromádka / National Museum / Prague, Czech Republic [p] // PARATYPE / PHILONTHUS (s. str.) / hapalemur sp. n. / Hromádka, det. 2008. [p, orange label]'.

Current status. Valid species.

Philonthus havai Hromádka, 2013

Philonthus havai Hromádka, 2013d: 396, Figs 45-46.

The holotype is deposited in NMPC (general collection): HoLOTYPE ( 3$)$ : ‘ $\delta$ [p, red label] // ETHIOPIA - Bale / Sabsebe Washa N Park $/ 07^{\circ} 03^{\prime} \mathrm{N} 39^{\circ} 39 \mathrm{E}$ [sic!] / $3.600 \mathrm{~m} 1971$ [p] // ex coll. L. Hromádka / National Museum / Prague, Czech Republic [p] // HOLOTYPUS / PHILONTHUS (s. str.) / havai sp. nov. / Hromádka, det., 2010. [p, orange label]'.

Current status. Valid species.

\section{Philonthus heliosciurus Hromádka, 2013}

Philonthus heliosciurus Hromádka, 2013g: 705, Figs 3-5.

The holotype is deposited in NMPC (general collection): HоLотуре ( $\left.{ }^{\Uparrow}\right)$ : ‘ 0 [p, pink label] // République Centraafricante [sic!] / Bozo lumiere / 21. v. 1981. / leg. N. Degallier [p] // ex coll. L. Hromádka / National Museum / Prague, Czech Republic [p] // HOLOTY PUS / PHILONTHUS (s. str.) / heliosciurus sp. nov. / Hromádka, det. 2012. [p, orange label]'.

Current status. Valid species.

Philonthus hippopotamus Hromádka, 2010

Philonthus hippopotamus Hromádka, 2010e: 29, Figs 81-83.

The holotype is deposited in NMPC (general collection): Holotype ( $\delta): ~ ‘[p$, red label] // ZIMBABWE / loc. Kutsaga / near Harare airport / 18.vi.1997 W. Rossi leg. [p, yellow label] // ex coll. L. Hromádka / National Museum / Prague, Czech Republic [p] // HOLOTYPUS / PHILONTHUS(Pseudophilo.) / hippopotamus spec. nov. / Hromádka, det.2008. [p, red label]'.

Current status. Valid species.

Philonthus hornaditanus Rambousek, 1925

Philonthus hornaditanus Rambousek, 1925: 75.

The lectotype and five paralectotypes are deposited in NMPC (general collection):

LectotyPe (đ): ‘Argent.Jujuy / Ornadito 3600 / XI.19. Weiser [p, green label] // TYPUS [p, red label] // hornaditanus // Bh.vid. Rmbk [hw] // Lectotype Philonthus / hornaditanus Rambousek, 1925 / des.Chani Posse de Maus 2008 [p, red label]'.
Paralectotype (ð): ‘Argent.Hor-/ nadita 3400 / XI.20.Weiser [p, green label] // TYPUS [p, red label] // hornaditanus // Bh.vid. Rmbk [hw] // Paralectotype Philonthus / hornaditanus Rambousek, 1925 / des. Chani Posse de Maus 2008 [p, red label]'.

PARAleCtOTYPes (2 unsexed specimens): 'Argent.Hor- / nadita 3400 / XI.20 Weiser [p, green label] // Paralectotype Philonthus / hornaditanus Rambousek, 1925 / des.Chani Posse de Maus 2008 [p, red label]'.

Paralectotypes ( 2 unsexed specimens on a same pin): 'Argent.Hor- $/$ nadita 3400 / XI.20 Weiser [p, green label] // Paralectotype Philonthus / hornaditanus Rambousek, 1925 / des.Chani Posse de Maus 2008 [p, red label] // Paralectotype Philonthus / hornaditanus Rambousek, 1925 / des.Chani Posse de Maus 2008 [p, red label]'.

Current status. Gabrius hornaditanus (Rambousek, 1925), see Chani-Posse de Maus (2009).

Comments. The lectotype was designated by CHANI-Posse DE MAUS (2009).

Philonthus hydrocynus Hromádka, 2012

Philonthus hydrocynus Hromádka, 2012d: 84, Figs 97-98.

The holotype is deposited in NMPC (general collection): Holotype (đ): 'République Centraafricante [sic!] / Bozo lumiere / 21. v. 1981 / leg. N. Degallier [p] // ex coll. L. Hromádka / National Museum / Prague, Czech Republic [p] // HOLOTYPUS / PHILONTHUS (s. str.) / hydrocynus spec.nov. / Hromádka, det., 2011. [p, orange label]'.

Current status. Valid species.

\section{Philonthus hylaeus Smetana, 1995}

Philonthus hylaeus Smetana, 1995: 399, Figs 634-639.

Two paratypes are deposited in NMPC (ex coll. L. Hromádka):

PARATYPeS (2 unsexed specimens): 'La. Concordia / Pa. 5 mi W Fer- / riday 2.V.76 / A. Smetana [p] // ex coll. L. Hromádka / National Museum / Prague, Czech Republic [p] // PARATYPE / Philonthus / hylaeus / A. Smetana 1993 / CNC No.21969 [p, yellow label]'

Current status. Valid species, see Herman (2001b).

Philonthus hyperolius Hromádka, 2012

Philonthus hyperolius Hromádka, 2012f: 59, Figs 13-15.

The holotype and nine paratypes are deposited in NMPC (general collection and ex coll. L. Hromádka):

HoLotype (đ): 'Kenya / Ngulia Lodge / 10/3 70 Palm [p] // ex coll. L. Hromádka / National Museum / Prague, Czech Republic [p] // HOLOTY PUS / PHILONTHUS (s.str.) / hyperolius sp. nov / Hromádka, det. 2011. [p, red label]'.

ParatyPes ( $\delta, 8$ unsexed specimens): 'KENYA/ Shimba For. / 17. 3. 1970 / T. Palm, leg [p, violet label] // ex coll. L. Hromádka / National Museum / Prague, Czech Republic [p] // PAR ATY PUS / PHILONTHUS (s.str.) / hyperolius sp. nov / Hromádka, det. 2011. [p, red label]'.

Current status. Valid species.

\section{Philonthus inachus Smetana, 1995}

Philonthus inachus Smetana, 1995: 103, Figs 71-76.

One paratype is deposited in NMPC (ex coll. L. Hromádka): PARATYPe (unsexed specimen): 'AZ. Chiricahua / Mts. Rustler Park / 8400' 26-27.VII. / 1979 A. Smetana [p] // ex coll. L. Hromádka / National Museum / Prague, Czech Republic [p] // PARATYPE / Philonthus / inachus / A. Smetana 1993 / CNC No.21949 [p, yellow label]'.

Current status. Valid species, see Herman (2001b). 
Philonthus indicator Hromádka, 2011

Philonthus indicator Hromádka, 2011a: 198, Figs 22-24.

The holotype is deposited in NMPC (general collection):

Holotype (ð): ‘ABESSIN. / Kristensen [p] // ex coll. L. Hromádka / National Museum / Prague, Czech Republic [p] // HOLOTYPUS / PHILONTHUS (s. str) / indicator sp. nov. / Hromádka, det. 2009 [p, orange label]'.

Current status. Valid species.

\section{Philonthus jaculus Hromádka, 2013}

Philonthus jaculus Hromádka, 2013e: 219, Figs 46-48.

The holotype is deposited in NMPC (general collection): Holotype ( $\left.)^{\prime}\right)$ : 'REP.SOUTH AFRICA:Natal / Cathedral Peaks For. / Sta.,75km WSW Estcourt / 7-31.XII.1979 ,S\&J Peck [p] // Ber 4,13.XII.79 / Bothas Shelter / Hyrax (Procavia) dung, 1760m [p] // ex coll. L. Hromádka / National Museum / Prague, Czech Republic [p] // HOLOTYPUS / PHILONTHUS (s. str.) / jaculus sp.nov. / Hromádka, det. 2009. [p, orange label]'.

Current status. Valid species.

\section{Philonthus janus Smetana, 1995}

Philonthus janus Smetana, 1995: 324, Figs 490-495.

Two paratypes are deposited in NMPC (ex coll. L. Hromádka):

Paratypes (2 unsexed specimens): 'ONT. Rondeau Pr. / Park South Pt. / Trail 2.VI.1985 / A.Smetana [p] // ex coll. L. Hromádka / National Museum / Prague, Czech Republic [p] // PARATYPE / Philonthus / janus / A. Smetana 1993 / CNC No.21960 [p, yellow label]'.

Current status. Valid species, see HERMAN (2001b).

\section{Philonthus jelinekianus Hromádka, 2009}

Philonthus jelinekianus Hromádka, 2009a: 674, Figs 8-12.

One paratype is deposited in NMPC (ex coll. L. Hromádka):

PARATyPe (ठ): 'Umuahia / J.L.GREGORY / 1960 - 15-vii [hw] // C.E. Tottenham / collection. / B.M. 1974-587. // ex coll. L. Hromádka/ National Museum/Prague, Czech Republic [p] // PARATYPUS / PHILONTHUS \{s. str.) / jelinekianus sp. nov. / Hromádka det. 2008. [p, red label]'.

Current status. Valid species.

Philonthus jureceki Boháč \& Hromádka, 1977

Philonthus jureceki Boháč \& Hromádka, 1977: 283, Figs 1-3.

The holotype and four paratypes are deposited in NMPC (general collection and ex coll. L. Hromádka):

Holotype (ð): 'Bulgaria occ. / Kňaževo / Š.Jureček lgt. [hw] // Holotypus [p, red label] // Philonthus / jureceki m. [hw] / J.Boháč det.19 [p] 76 [hw]'.

Paratype ( $\hat{\sigma}$, aedeagus mounted on separate card): 'Bulgaria occ. $/$ Kňaževo / Š.Jureček lgt. [hw] // Paratypus [p, red label] // Philonthus / jureceki m. [hw] / J.Boháč det.19 [p] 76 [hw]'.

Paratype ( + ): ‘ + [hw] // Bulgaria occ. / Kn̆aževo / Š.Jureček lgt. [hw] // Paratypus [p, red label] // Philonthus / jureceki m. [hw] / J.Boháč det.19 [p] $76[\mathrm{hw}]^{\prime}$.

Paratypes (1 $\delta, 1$ unsexed specimen): 'Bulgaria 16.3.09 / Kňaževo Š. Jureček lgt. [hw, blue label] // ex coll. L. Hromádka / National Museum / Prague, Czech Republic [p] // PHILONTHUS (s. str.) / jurecekianus / sp. n. / Boháč et Hromádka, 1977 [p, orange label] // Paratypus [p, red label]'.

Current status. Philonthus jurecekianus Boháč \& Hromádka, 1980, see SchüLKE \& SMETANA (2015).
Comments. BoHÁČ \& HromÁDKA (1980) proposed the new substitute name Philonthus jurecekianus Boháč \& Hromádka, 1980 for Philonthus jureceki Boháč \& Hromádka, 1977, a junior secondary homonym of Philonthus jureceki (Rambousek, 1913).

\section{Philonthus khouzestanicus Boháč, 1981}

Philonthus knouzestanicus [sic!] Boháč, 1981: 356, Figs 3, 4.

Holotype and six paratypes are deposited in NMPC (general collection and ex coll. L. Hromádka):

Holotype (ð): ‘SE Iran / Tis / 6.-7. 4.1973 [p] // Loc. no. 150 / Exp. Nat. Nus. [sic!] / Praha [p] // Holotypus [p, red label] // Philonthus m. / khouzestanicus [hw]/ J.Boháč det. $19[\mathrm{p}] 76[\mathrm{hw}]$ '.

ParatyPes (5 unsexed specimens): 'SE Iran / Tis / 6.-7. 4. 1973 [p] // Loc. no. 150 / Exp. Nat. Nus. [sic!] / Praha [p] // Paratypus [p, red label]'. ParaTYPE (unsexed specimen): 'SE Iran / Tis / 6.-7. 4. 1973 [p] // Loc. no. 150 / Exp. Nat. Nus. [sic!] / Praha [p] // ex coll. L. Hromádka / National Museum / Prague, Czech Republic [p] // Paratypus [p, red label] // Philonthus m. / khouzestanicus [hw] / J.Boháč det. 19 [p] 82 [hw]'.

Current status. Valid species, see SchülKe \& SMETANA (2015).

Philonthus kobus Hromádka, 2010

Philonthus kobus Hromádka, 2010e: 30, Figs 84-87.

The holotype is deposited in NMPC (general collection): HoLOTYPe (ð): ‘ ‘ [p, green label] // Afrique or allemande / KILIMANDJARO / VERSANT SUD-EST / ALLUAUD \& JEANNEL [p] // ex coll. L. Hromádka / National Museum / Prague, Czech Republic [p] // HOLOTYPUS / PHILONTHUS (Pseudophil.) / kobus sp. nov. / Hromádka, det. 2008 [p, orange label]'.

Current status. Valid species.

Philonthus krali Hromádka, 1987

Philonthus krali Hromádka, 1987b: 147, Figs 1-4.

The holotype is deposited in NMPC (ex coll. L. Hromádka): HoLOTYPe (đ̋): 'USSR Uzbekistan / Aktaš 1100-1300m / /Taškent env./ / 4.VII.1976 / Josef Král lgt. [p] // ex coll. L. Hromádka / National Museum / Prague, Czech Republic [p] // HOLOTY PUS / Philonthus / krali sp. n. / L.Hromádka, det. 1987 [p]’.

Current status. Valid species, see SchülKe \& SMETANA (2015).

Philonthus kralianus Hromádka, 1987

Philonthus kralianus Hromádka, 1987b: 149, Figs 5-7.

The holotype is deposited in NMPC (ex coll. L. Hromádka): HoLOTYPE (đ): 'USSR Tadžikistan / Ziddy 26.6.1976 / Hissar.chr.2100m / Josef Král lgt. [p] // ex coll. L. Hromádka / National Museum / Prague, Czech Republic [p] // HOLTYPUS [sic!] / Philonthus / kralianus sp. n. / L.Hromádka, det. 1987. [p, orange label] // Philonthus / liopterus SHARP / det. Schillhammer 1999 [p]'.

Current status. Junior subjective synonym of Philonthus liopterus Sharp, 1889, see Schillhammer (1999) and SCHÜLKe \& SMETANA (2015).

\section{Philonthus labeo Hromádka, 2011}

Philonthus labeo Hromádka, 2011c: 172, Figs 43-47.

The holotype is deposited in NMPC (general collection): HoLOTYPE (ð): 'Transvaal : Soutpansberg / distr., Hanglipbos Forest / 
brouss. dégr. (ZA. 7) [p] // ex coll. L. Hromádka / National Museum / Prague, Czech Republic [p] // HOLOTYPUS / PHILONTHUS (s. str.) / labeo sp. nov. / Hromádka, det. 2009. [p, orange label]'

Current status. Valid species.

Philonthus lagonosticta Hromádka, 2010

Philonthus lagonosticta Hromádka, 2010e: 33, Figs 88-92.

One paratype is deposited in NMPC (ex coll. L. Hromádka): PARATYPE (ठ̋): ‘728 [p, pink label] // Eboufek. / 28.vii. [sic!] 1993 / FITrap 1. [p] // CAMEROON: / Mbalmayo F. Res. / TIGER Survey. / BM 1994-148. [p] // 155 [hw] // ex coll. L. Hromádka / National Museum / Prague, Czech Republic [p] // PARATY PUS / PHILONTHUS (Pseudoph.) / lagonosticta sp. nov. / Hromádka, det. 2008. [p, pink label]'.

Current status. Valid species.

Philonthus lamtoensis shamnesis Hromádka, 2005

Philonthus (Raucalius) lamtoensis shamnesis Hromádka, 2005: 113.

The holotype is deposited in NMPC (ex coll. L. Hromádka):

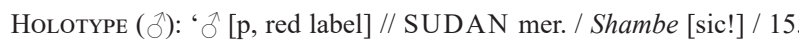
10. 1966 / Dr. P. Štys leg. [p, green label] // ex coll. L. Hromádka / National Museum / Prague, Czech Republic [p] // HOLOTY PUS / PHILONTHUS(Raucalius) / lamtoensis / ssp. shamnesis sp. n. / L. Hromádka, det. 2004 [p, yellow label]'.

Current status. Valid subspecies, see HromádKa (2008b).

\section{Philonthus lan Schillhammer, 1998}

Philonthus lan Schillhammer, 1998: 107, Figs 9, 10, 19.

Three paratypes are deposited in NMPC (ex coll. L. Hromádka):

PARATYPe ( $\precsim$ ): ‘ $\precsim$ [p] // CHINA-YUNNAN / 28.5.-9.6. 1994 DALI / lgt. E. Kučera [p] // ex coll. L. Hromádka / National Museum / Prague, Czech Republic [p] // PARATYPE / Philonthus / lan sp.n. / des. Schillhammer 1997 [p, orange label]'.

PARATYPes (2 unsexed specimens): 'CHINA-YUNNAN / 28.5.-9.6. 1994 DALI / lgt. E. Kučera [p] // ex coll. L. Hromádka / National Museum / Prague, Czech Republic [p] // PARATYPE / Philonthus / lan sp.n. / des. Schillhammer 1997 [p, orange label]'.

Current status. Valid species, see SchüLKE \& SMETANA (2015).

\section{Philonthus lanius Hromádka, 2013}

Philonthus lanius Hromádka, 2013c: 196, Figs 33-37.

The holotype and two paratypes are deposited in NMPC (ex coll. L. Hromádka):

Holotype (ठ)): 'Kamerun / Joh.-Albrechtshöhe / 14.IX.-6.X.98 / 1.Conradt S. [p] // ex coll. L. Hromádka / National Museum / Prague, Czech Republic [p] // HOLOTYPUS / PHILONTHUS (s. str.) / lanius sp.nov. / Hromádka, det., 2013. [p, red label]'.

PARATyPes $(2 \delta)$ : 'Kamerun [hw] // ex coll. L. Hromádka / National Museum / Prague, Czech Republic [p] // PARATY PUS / PHILONTHUS (s. str.) / lanius sp.nov. / Hromádka, det., 2013. [p, red label]'.

Current status. Valid species.

\section{Philonthus lecoqi Janák, 2013}

Philonthus lecoqi Janák, 2013: 24, Figs 1-11.

One paratype is deposited in NMPC (ex coll. L. Hromádka):

PARATYPe ( đ̋): 'CASENT / 8037478 [p] // MADAGASCAR: Province / Diego-Suarez, Montaigne / Francais, elev 150 m / 15 Feb - 6 March $2001[\mathrm{p}] / / 12^{\circ} 18^{\prime} 8^{\prime \prime} \mathrm{S}, 49^{\circ} 38^{\prime}$ 51" E / R. Harin'Hala coll. malaise / along forested limestone / ridge MA-01-06-06 [p] // ठ [p]// ex coll. L. Hromádka / National Museum / Prague, Czech Republic [p] // PARATYPUS / Philonthus / lecoqi sp. n. / J. Janák det. 2012 [p, red label]'.

Current status. Valid species.

Philonthus lephiobagrus Hromádka, 2013

Philonthus lephiobagrus Hromádka, 2013b: 7, Figs 15-17.

The holotype is deposited in NMPC (general collection): Holotype (đ): 'TANZANIA, Arusha / 6.-7.4. 1997,Mt.Meru / 18002000 m., Kuboň lt. [p] // ex coll. L. Hromádka / National Museum / Prague, Czech Republic [p] // HOLOTYPUS / PHILONTHUS (s. str.) / lephiobagrus sp. nov. / Hromádka, det., 2012. [p, orange label]'.

Current status. Valid species.

Philonthus leptophilus Hromádka, 2006

Philonthus (s. str.) leptophilus Hromádka, 2006: 65, Figs 1-8.

Two paratypes are deposited in NMPC (ex coll. L. Hromádka):

PARATYPe ( 0 , dissected genitalia on a separate plastic card): 'SIERRA LEONE / Western Area / Freetown Faurah Bay / College Botanical reserve / 9.xii.1992 W. Rossi leg [p, yellow label] // ex coll. L. Hromádka / National Museum / Prague, Czech Republic [p] // PARATYPUS / PHILONTHUS (s.str.) / leptoptilus sp. nov. §/ Hromádka, det.2005. [p, red label]'.

PARATYPe ( + ): 'SIERRA LEONE / Western Area / Freetown Faurah Bay / College Botanical reserve / 9.xii.1992 W. Rossi leg [p, yellow label] // ex coll. L. Hromádka / National Museum / Prague, Czech Republic [p] // PARATYPUS / PHILONTHUS (s.str.) / leptoptilus sp. nov. $q$ / Hromádka, det.2005. [p, red label]'.

Current status. Valid species, see HromádKa (2009c). Comments. Two original spellings, P. leptoptilus (p. 65, 67-68) and P. leptophilus (p. 68), are mentioned in the original description (the species was named after the Marabou stork Leptoptilos crumenifer). HROMÁDKA (2009c: 180) fixed the correct spelling $P$. leptophilus as the first reviser (see ICZN 1999: Article 24.2.4).

Philonthus litocranius Hromádka, 2013

Philonthus litocranius Hromádka, 2013b: 8, Figs 18-20.

The holotype and one paratype are deposited in NMPC (general collection):

Holotype (ð): 'République Centraafricante [sic!] / Bozo lumiere / 21. v. 1981 / leg. N. Degallier [p] // ex coll. L. Hromádka / National Museum / Prague, Czech Republic [p] // HOLOTYPUS / PHILONTHUS (s. str.) / litocranius sp.nov. / Hromádka, det. 2012. [p, red label]'.

PARAType (ð): 'République Centraafricante [sic!] / Bozo lumiere / 21. v. 1981 / leg. N. Degallier [p] // ex coll. L. Hromádka / National Museum / Prague, Czech Republic [p] // PARAT Y PUS / PHILONTHUS (s. str.) / litocranius sp.nov. / Hromádka, det. 2012. [p, red label]'.

Current status. Valid species.

Philonthus loxodonta Hromádka, 2009

Philonthus loxodonta Hromádka, 2009a: 675, Figs 13-19.

Two paratypes are deposited in NMPC (ex coll. L. Hromádka):

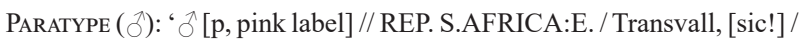
Pilgrims rest, / (19-31)-XII-1985 [p] // FMHD \#85-860, / relict nature / for.dung trps,S. [sic!] / Peck P\# 85-293 [p] // ex coll. L. Hromádka 
/ National Museum / Prague, Czech Republic [p] // PARATY PUS / PHILONTHUS (s. str.) / loxodonta sp. nov. / Hromádka, det. 2006 [p, red label]'.

PARATYPE ( $\precsim$ ): 'REP. S.AFRICA:E. / Transvall, [sic!] / Pilgrims rest, / (1931)-XII-1985 [p] // FMHD \#85-860, / relict nature / for.dung trps,S. [sic!] / Peck P\# 85-293 [p] // ex coll. L. Hromádka / National Museum / Prague, Czech Republic [p] // PARATYPUS / PHILONTHUS (Philonth.) / loxodonta sp. nov. / Hromádka, det, 2007 [p, red label]'.

Current status. Valid species.

\section{Philonthus lybius Hromádka, 2013}

Philonthus lybius Hromádka, 2013d: 405, Figs 70-72.

The holotype and four paratypes are deposited in NMPC (general collection and ex coll. L. Hromádka):

HoLotyPe (ð): 'ABESSIN. / Kristensen [p] // ex coll. L. Hromádka / National Museum / Prague, Czech Republic [p] // HOLOTYPUS / PHILONTHUS (s. str.) / lybius sp. nov. / Hromádka, det. 2009. [p, red label]'.

ParatyPes (2 ô): 'ABESSIN. / Kristensen [hw] // ex coll. L. Hromádka / National Museum / Prague, Czech Republic [p] // PARATYPUS / PHILONTHUS (s. str.) / lybius sp. nov. / Hromádka, det. 2009. [p, red label]'.

ParatyPes $(2$ ô): ‘ABESSIN. / Kristensen [p] // ex coll. L. Hromádka / National Museum / Prague, Czech Republic [p] // PARATYPUS / PHILONTHUS (s. str.) / lybius sp. nov. / Hromádka, det. 2009. [p, red label]'.

Current status. Valid species.

\section{Philonthus mabuya Hromádka, 2013}

Philonthus mabuya Hromádka, 2013d: 406, Figs 73-75.

The holotype is deposited in NMPC (general collection):

HoLotype ( $\left.\jmath^{\jmath}\right)$ : 'KENYA / M. Elgon 2380 m. Reinhardt leg. [p] // ex coll. L. Hromádka / National Museum / Prague, Czech Republic [p] // HOLOTYPUS / PHILONTHUS (s. str.) / mabuya spec. nov. / Hromádka det. 2009. [p, orange label]'.

Current status. Valid species.

Philonthus macronectes Hromádka, 2015

Philonthus macronectes Hromádka, 2015a: 62, Figs 6-9.

Two paratypes are deposited in NMPC (ex coll. L. Hromádka):

PARATYPe ( $)$ : 'TANZANIA 2434m / Mount Hanang / S04²4'41"; E35²4'10" / 25-28.v.12 Dung Pitfall / leg. Smith,R \& Takano,H [p] // BMNH \{E\} / 2012-92 [p] // BMNH (E) / 1262412 [p] // ex coll. L. Hromádka / National Museum / Prague, Czech Republic [p] // PARATYPUS / PHILONTHUS (s. str.) / macronectes sp.nov. / Hromádka, det., 2014. [p, red label]'.

Paratype (ठ): 'TANZANIA 2434m / Mount Hanang / S04²4'41"; E35 24'10" / 25-28.v.12 Dung Pitfall / leg. Smith,R \& Takano,H [p] // BMNH $\{\mathrm{E}\} / 2012-92$ [p] // BMNH (E) / 1262402 [p] // ex coll. L. Hromádka / National Museum / Prague, Czech Republic [p] // PARATYPUS / PHILONTHUS (s. str.) / macronectes sp.nov. / Hromádka, det., 2014. [p, red label]'.

Current status. Valid species.

Philonthus macronyx Hromádka, 2010

Philonthus macronyx Hromádka, 2010c: 43, Figs 53-56.

Seven paratypes are deposited in NMPC (ex coll. L. Hromádka):

Paratypes (1 $\hat{\delta}, 1$ unsexed specimen): 'I.R.S.A.C.-MUS.CONGO /
Kundelungu 1750 m. [p] / 9-III [hw] -1950 / N. Leleup [p] / (Prairie herb.) [hw] // Récolté dans / nid de / Cryptomys [p, green label] // ex coll. L. Hromádka / National Museum / Prague, Czech Republic [p] // PARATY PUS / PHILONTHUS (Philonth.) / macronyx sp. nov. / Hromádka, det. 2008. [p, red label]'.

PARATYPES (2 unsexed specimens): 'I.R.S.A.C.-MUS.CONGO / Kundelungu 1750 m. [p] / (Prairie herb.)9-III [hw] -1950 / N. Leleup [p] // Récolté dans / nid de / Cryptomys [p, green label] // ex coll. L. Hromádka / National Museum / Prague, Czech Republic [p] // PARATYPUS / PHILONTHUS (Philont.) / macronyx sp. nov. / Hromádka, det. 2008 [p, red label]'.

Paratype (ð): 'COLL. MUS. CONGO / Massif des Kunde- / lungu [p] air. $1750 \mathrm{~m}$. [hw] / N. Leleup [p] / (Prairie tourbeuse) 3-III-1950 [sic!] [hw] // Récolté dans [p] / terrier de / Cryptomys [hw, green label] // ex coll. L. Hromádka / National Museum / Prague, Czech Republic [p] // PARATY PUS / PHILONTHUS (Philont.) / macronyx sp. nov. / Hromádka, det. 2008 [p, red label]'.

ParatyPe (unsexed specimen): 'I.R.S.A.C.-MUS.CONGO / Kundelungu 1750 m. [p] / III [hw] -1950 / N. Leleup [p] / (Prairie marecag.) [hw] / M.C.3 [hw] // Récolté dans / nid de / Cryptomys [p, green label] // ex coll. L. Hromádka / National Museum / Prague, Czech Republic [p] // PARATY PUS / PHILONTHUS (Philont.) / macronyx sp. nov. / Hromádka, det. 2008 [p, red label]'.

PARATYPE (unsexed specimen): 'I.R.S.A.C.-MUS.CONGO / Kundelungu 1750 m. [p] / III [hw] -1950 / N. Leleup [p] / (Prairie marecag.) [hw] / M.C.3 [hw] // Récolté dans / nid de / Cryptomys [p, green label] // Voir ladulte et / larves en alcool [hw] // ex coll. L. Hromádka / National Museum / Prague, Czech Republic [p] // PARATYPUS / PHILONTHUS (Philont.) / macronyx sp. nov. / Hromádka, det. 2008 [p, red label]'.

Current status. Valid species.

Philonthus mandrillus Hromádka, 2013

Philonthus mandrillus Hromádka, 2013f: 715, Figs 6-7.

One paratype is deposited in NMPC (ex coll. L. Hromádka):

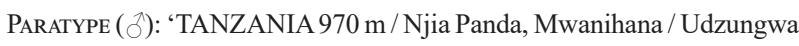
Mountains N.P / S0747'27.7";E03649'33.7" / 12-14.iv.11 Dung Pitfall Trap / leg. Smith,R \& Takano,H [p] // BMNH $\{$ E $\} / 2011-88$ [p] // ex coll. L. Hromádka / National Museum / Prague, Czech Republic [p] // PARATYPUS / PHILONTHUS (s. str.) / mandrillus (s. str.) / Hromádka, det., 2012. [p, orange label]'.

Current status. Valid species.

\section{Philonthus mehelya Hromádka, 2011}

Philonthus mehelya Hromádka, 2011e: 187, Figs 38-40.

Three paratypes are deposited in NMPC (ex coll. L. Hromádka):

PARATyPes (2 $\delta 1$ o): 'Congo Belge / Libenge.12.i.1948 / R.Cremer M.Neuman / ds excrém.humains [p] // R.I.Sc.Nat.Belg. / I. G. 16.665 [p] // ex coll. L. Hromádka / National Museum / Prague, Czech Republic [p] // PARATYPUS / PHILONTHUS (s.str.) / mehelya sp. nov. / Hromádka, det. 2010. [p, orange label]'.

Current status. Valid species.

Philonthus melaenornis Hromádka, 2011

Philonthus melaenornis Hromádka, 2011a: 200, Figs 29-32.

One paratype is deposited in NMPC (ex coll. L. Hromádka):

PARATYPE (đ): 'I.R.S.A.C.-MUS.R.A.C. / S. Rhodesia : Chipinga, [sic!] / 1200 à 1300 m. / VII.1960 N. Leleup // Humus dans résidu / forêt ombrophile [p] // ex coll. L. Hromádka / National Museum / Prague, Czech Republic [p] // PARATYPUS / PHILONTHUS (s. str.) / melaenornis sp. nov. / Hromádka, det., 2011. [p, orange label]'.

Current status. Valid species. 
Philonthus merops Hromádka, 2011

Philonthus merops Hromádka, 2011g: 1346, Figs 1-4.

Seven paratypes are deposited in NMPC (ex coll. L. Hromádka):

PARATYPe (đ): 'GHANA: Ashanti region / Kwadaso / 320 m,N 642 W 139 / Dr. S. ENDRÖDY-YOUNGA [p] // Nr.331 / black light / 31.III.1969 [p] // ex coll. L. Hromádka / National Museum / Prague, Czech Republic [p] // PARATYPUS / PHILONTHUS (s. str.) / merops sp. nov. / Hromádka, det. 2011. [p, red label] // PARATYPUS / PHILONTHUS (s. str.) / hromadkai sp. nov. / Schülke, 2011. [p, red label]'.

PARATYPE (む): 'GHANA: Ashanti region / Kumasi, Nhiasu / 330 m,N 6 43 - W 136 / Dr. S. ENDRÖDY-YOUNGA [p] // Nr.280 / at light / 9.X.1967 [p] // ex coll. L. Hromádka/ National Museum/ Prague, Czech Republic [p] // PARATYPUS / PHILONTHUS (s. str.) / merops sp. nov. / Hromádka, det. 2011. [p, red label] // PARATYPUS / PHILONTHUS (s. str.) / hromadkai sp. nov. / Schülke, 2011 [p, red label]'.

PARATYPe (unsexed specimen): 'GHANA:Northern region / BandaNkwanta / 150 m,N 822 - W 208 / Dr. S. ENDRÖDY-YOUNGA [p] // Nr.47 / light trap / 26-31.VII.1965 [p] // ex coll. L. Hromádka / National Museum / Prague, Czech Republic [p] // PARATY PUS / PHILONTHUS (s. str.) / merops sp. nov. / Hromádka, det. 2011. [p, red label] // PARATY PUS / PHILONTHUS (s. str.) / hromadkai sp. nov. / Schülke, 2011. [p, red label]'.

Paratype ( ): 'GHANA: Ashanti region / Kwadaso / 320 m,N 642 W 139 / Dr. S. ENDRÖDY-YOUNGA [p] // Nr.327 / mixed light / 18.III.1969 [p] // ex coll. L. Hromádka / National Museum / Prague, Czech Republic [p] // PARATYPUS / PHILONTHUS (s. str.) / merops sp. nov. / Hromádka, det. 2011. [p, red label] // PARATYPUS / PHILONTHUS (s. str.) / hromadkai sp. nov. / Schülke, 2011. [p, red label]'.

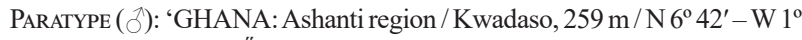
39' / Dr. S. ENDRÖDY-YOUNGA [p] // Nr.398 / on light / 15.IX.1969. [p] // ex coll. L. Hromádka / National Museum / Prague, Czech Republic [p] // PARATYPUS / PHILONTHUS (s. str.) / merops sp. nov. / Hromádka, det. 2011. [p, red label] // PARATYPUS / PHILONTHUS (s. str.) / hromadkai sp. nov. / Schülke, 2011. [p, red label]'.

PARATYPE (unsexed specimen): 'WEST AFRICA,Ghana / Northern Region / Tamale / leg.Dr.S.Endrödi [p] // No. 51 / Lichtfalle/Quarz/ / 27.VIII.1970. [p] // ex coll. L. Hromádka / National Museum / Prague, Czech Republic [p] // PARATY PUS / PHILONTHUS (s. str.) / merops sp. nov. / Hromádka, det. 2011. [p, red label] // PARATYPUS / PHILONTHUS (s. str.) / hromadkai sp. nov. / Schülke, 2011. [p, red label]'.

Paratype ( $(3)$ : 'SIERRA LEONE / Western Area / Base Picket Hill / 9.i.1997 W.Rossi [p, yellow label] // ex coll. L. Hromádka / National Museum / Prague, Czech Republic [p] // PAR ATY PUS / PHILONTHUS (s. str.) / merops sp. nov. / Hromádka, det. 2011. [p, red label] // PARATYPUS / PHILONTHUS (s. str.) / hromadkai sp. nov. / Schülke, 2011. [p, red label]'.

Current status. Philonthus hromadkai Schülke, 2012. Comments. SCHÜLKE (2012) proposed the new substitute name Philonthus hromadkai Schülke, 2012 for Philonthus merops Hromádka, 2011, a junior primary homonym of Philonthus (Onychophilonthus) merops Smetana, 1963.

\section{Philonthus merorientis Smetana, 1995}

Philonthus merorientis Smetana, 1995: 408, Figs 652-657.

Two paratypes are deposited in NMPC (ex coll. L. Hromádka):

PARATYPES (2 unsexed specimens): 'FLA.L.Istokpoga n. / L.Placid, 7.III.68 / A. Smetana coll. [p] // ex coll. L. Hromádka / National Museum / Prague, Czech Republic [p] // PARATYPE / Philonthus / merorientis / A. Smetana 1993 / CNC No.21971 [p, yellow label]'.

Current status. Valid species, see Herman (2001b).
Philonthus mnemon Smetana, 1995

Philonthus mnemon Smetana, 1995: 157, Figs 163-170.

Two paratypes are deposited in NMPC (ex coll. L. Hromádka):

Paratype (unsexed specimen): 'AZ. Huachuca Mts. / Bear Saddle / 8100' 5.VIII.79/A. Smetana [p] // ex coll. L. Hromádka / National Museum / Prague, Czech Republic [p] // PARATYPE / Philonthus / mnemon / A. Smetana 1993 / CNC No.21950 [p, yellow label]'.

PARATYPE (unsexed specimen): 'ARIZ.Graham Co.Pina- / leno Mts.Wet Cn. / 6100’29.VII.69 / A.Smetana [p] // ex coll. L. Hromádka / National Museum / Prague, Czech Republic [p] // PARATYPE / Philonthus / mnemon / A. Smetana 1993 / CNC No.21950 [p, yellow label]'.

Current status. Valid species, see Herman (2001b).

\section{Philonthus monaeses Smetana, 1995}

Philonthus monaeses Smetana, 1995: 402, Figs 640-645.

Two paratypes are deposited in NMPC (ex coll. L. Hromádka):

PARATYPES (2 unsexed specimens): 'La. Concordia / Pa. 5 mi W Fer-/ riday 1.V.76 / A. Smetana [p] // ex coll. L. Hromádka / National Museum / Prague, Czech Republic [p] // PARATYPE / Philonthus / monaeses / A. Smetana 1993 / CNC No.21970 [p, yellow label]'.

Current status. Valid species, see Herman (2001b).

Philonthus mormyrops Hromádka, 2013

Philonthus mormyrops Hromádka, 2013e: 224, Figs 58-61.

The holotype and three paratypes are deposited in NMPC (ex coll. L. Hromádka):

Holotype (đ): 'MALAWI S / Mulanoe Mts. env. / 22. xii. 2001 / J. Bezděk, lgt. [p, yellow label] // ex coll. L. Hromádka / National Museum / Prague, Czech Republic [p] // HOLOTY PUS / PHILONTHUS (s. str.) / mormyrops spec. nov. / Hromádka, det. 2008 [p, red label]'. Paratype (+): 'MALAWI S / Mulanoe Mts. env. / 22. xii. 2001 / J. Bezděk, lgt. [p, yellow label] // ex coll. L. Hromádka / National Museum / Prague, Czech Republic [p] // PARATYPUS / PHILONTHUS (s. str.) / mormyrops spec. nov. / Hromádka, det. 2008 [p, red label]'.

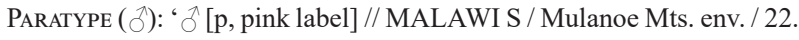
xii. 2001 / J. Bezděk, lgt. [p, yellow label] // ex coll. L. Hromádka / National Museum / Prague, Czech Republic [p] // PARATYPUS / PHILONTHUS (s. str.) / mormyrops spec. nov. / Hromádka, det. 2008 [p, pink label]'.

Paratype ( $\left.{ }^{1}\right)$ : 'MALAWI S Jall env. / $30 \mathrm{~km} \mathrm{SE} \mathrm{of} \mathrm{Zamba} \mathrm{/} \mathrm{26.-27.}$ xii.2001 / J. Bezděk lgt. [p, violet label] // ex coll. L. Hromádka / National Museum / Prague, Czech Republic [p] // PAR ATY PUS / PHILONTHUS (s. str.) / mormyrops sp. nov. / Hromádka, det., 2008 [p, red label]'.

Current status. Valid species.

\section{Philonthus nectarinia Hromádka, 2012}

Philonthus nectarinia Hromádka, 2012e: 183, Figs 15-21.

The holotype and three paratypes are deposited in NMPC (general collection):

HoLotype (đ̋): 'N. Rhodesia : Abercorn, / 1800 m. / VII.1960 [sic!] N. Leleup [p] // Galerie forestière / de la Mwengo, / dans l'humus [p] // ex coll. L. Hromádka / National Museum / Prague, Czech Republic [p] // HOLOTYPUS / PHILONTHUS (S. STR.) / nectarinia sp. nov. / Hromádka, det., 2011. [p, orange label]'.

Paratype (+): 'N. Rhodesia : Abercorn, / 1800 m. / VII.1960 [sic!] N. Leleup [p] // Galerie forestière / de la Mwengo, / dans l'humus [p] // ex coll. L. Hromádka / National Museum / Prague, Czech Republic [p] // PHILONTHUS (Philont.) / nairobianus / Cameron, 1950 / Hromádka, 
det.2007 [p] // PARATYPUS / PHILONTHUS(S. STR.) / nectarinia sp. nov. / Hromádka, det., 2011. [p, orange label]'.

Paratypes $(2 \delta$ ) : 'ETHIOPIA: Bale / 8km. W. of Dinshu / $0706 \mathrm{~N}$ 3944 E. / 3.050m. xii. 1971 [sic!] [p] // ex coll. L. Hromádka / National Museum / Prague, Czech Republic [p] // PARATYPUS / PHILONTHUS (S. STR.) / nectarinia sp. nov. / Hromádka, det., 2011. [p, orange label]'.

Current status. Valid species.

\section{Philonthus neptunus Smetana, 1995}

Philonthus neptunus Smetana, 1995: 436, Figs 700-706.

One paratype is deposited in NMPC (ex coll. L. Hromádka):

PARATYPe (unsexed specimen): 'USA:FL:Monroe Co / Middle Torch Key Sec.17 / Lazelle Place,uv light / trap,10-19.VIII.92 / S\&J Peck 92315 [p] // ex coll. L. Hromádka / National Museum / Prague, Czech Republic [p] // PARATYPE / Philonthus / neptunus / A. Smetana 1993 / CNC No.21975 [p, yellow label]'.

Current status. Valid species, see Herman (2001b).

\section{Philonthus numida Hromádka, 2013}

Philonthus numida Hromádka, 2013d: 412, Figs 88-90.

Two paratypes are deposited in NMPC (ex coll. L. Hromádka):

PARATYPe (ठ): ‘ABYSSINIA: / Gamo Prov., / Mt. Tola [Gughé / highlands], over 10,000ft. / 5.xii.1948 [p] // From track across / precipitous eastern / escarpment [p] // ex coll. L. Hromádka / National Museum / Prague, Czech Republic [p] // PARAT Y PUS / PHILONTHUS (s.str.) / numida sp. nov. [p] ? [hw] / Hromádka, det. 2006. [p, red label]'.

Paratype (ð): 'ABYSSINIA: / Gamo Prov., / Mt. Tola [Gughé / highlands], over 10,000ft. / 5.xii.1948 [p] // From track across / precipitous eastern / escarpment [p] // ex coll. L. Hromádka / National Museum / Prague, Czech Republic [p] // PARAT Y PUS / PHILONTHUS (s.str.) / numida sp. nov. / Hromádka, det. 2006. [p, red label]'.

Current status. Valid species.

Philonthus okapia Hromádka, 2010

Philonthus okapia Hromádka, 2010e: 38, Figs 103-106.

The holotype is deposited in NMPC (general collection): HoLOTYPe (§̋): ‘GABON, Belinga / 12.III.1963 (199 L) / Leg. H. Coiffait [p, blue label] // HOLOTYPUS / PHILONTHUS (Pseudo.) / okapi [sic!] spec. nov. / Hromádka, det. 2008 [p, orange label]'.

Current status. Valid species.

\section{Philonthus osteolaemus Hromádka, 2010}

Philonthus osteolaemus Hromádka, 2010d: 105, Figs 20-23.

The holotype and one paratype are deposited in NMPC (general collection and ex coll. L. Hromádka):

HoLOTYPe ( $\delta$, dissected genitalia on a separate plastic card): 'REP. SOUTH Africa / Nothern Prov. Camp / David, $5 \mathrm{~km} \mathrm{~S} \mathrm{Ofoolaco} \mathrm{/}$ 475m 17.-24.i.2002 [sic!] / leg. S. Murzin [p] // ex coll. L. Hromádka / National Museum/ Prague, Czech Republic [p] // HOLOTYPUS / PHILONTHUS (Philonthus) / osteolaemus sp. nov. / Hromádka, det. 2007 [p, orange label]'.

PARATYPe ( + ): 'REP.SOUTH Africa / Nothern Prov. Camp / David, 5 km S Ofoolaco / 475m 17.-24.i.2002 [sic!] / leg. S. Murzin [p] // ex coll. L. Hromádka / National Museum / Prague, Czech Republic [p] // PARATYPUS / PHILONTHUS (Philonthus) / osteolaemus sp. nov. / Hromádka, det. 2007 [p, orange label]'.

Current status. Valid species.
Philonthus pan Hromádka, 2008

Philonthus (s. str.) pan Hromádka, 2008c: 217, Figs 24-28.

Thirty eight paratypes are deposited in NMPC (ex coll. L. Hromádka):

Paratypes (3 30 ): 'MALAWI S / Mulanoe Mts. env. / 22. xii. 2001 / J. Bezděk, lgt. [p, yellow label] // ex coll. L. Hromádka / National Museum / Prague, Czech Republic [p] // PARATYPUS / PHILONTHUS (s. str.) / pan sp.n. J / L. Hromádka, det. 2004 [p, orange label]'.

PARATYPE ( + , dissected genitalia on a separate plastic card): 'MALAWI S / Mulanoe Mts. env. / 22. xii. 2001 / J. Bezděk, lgt. [p, yellow label] // ex coll. L. Hromádka / National Museum / Prague, Czech Republic [p] // PARATYPUS / PHILONTHUS (s. str.) / pan sp.n. + / L. Hromádka, det. 2004 [p, orange label]'.

Paratypes (5 9 ) : 'MALAWI S / Mulanoe Mts. env. / 22. xii. 2001 / J. Bezděk, lgt. [p, yellow label] // ex coll. L. Hromádka / National Museum / Prague, Czech Republic [p] // PARATYPUS / PHILONTHUS (s. str.) / pan sp.n. + / L. Hromádka, det. 2004 [p, orange label]'.

Paratypes (2 d): 'MALAWI S / Balaka / 19. xii. 2001 / J. Bezděk, lgt. [p, yellow label] // ex coll. L. Hromádka / National Museum / Prague, Czech Republic [p] // PARATYPUS / PHILONTHUS (s. str.) / pan sp.n. § / L. Hromádka, det. 2004 [p, orange label]'.

PARATYPe ( 3 ): ‘ [p green label] // MALAWI S / Balaka / 19. xii. 2001 / J. Bezděk, lgt. [p, yellow label] // ex coll. L. Hromádka/ National Museum / Prague, Czech Republic [p] // PARATYPUS / PHILONTHUS (s. str.) / pan sp.n. J / L. Hromádka, det. 2004 [p, orange label]'.

ParatyPes (2 + + ): 'MALAWI S / Balaka / 19. xii. 2001 / J. Bezděk, lgt. [p, yellow label] // ex coll. L. Hromádka / National Museum / Prague, Czech Republic [p] // PARATYPUS / PHILONTHUS (s. str.) / pan sp.n. † / L. Hromádka, det. 2004 [p, orange label]'.

PARATYPE ( + ): ‘ $\odot$ [p, orange label] // MALAWI S / Balaka / 19. xii. 2001 / J. Bezděk, lgt. [p, yellow label] // ex coll. L. Hromádka/ National Museum / Prague, Czech Republic [p] // PARATYPUS / PHILONTHUS (s. str.) / pan sp.n. / / L. Hromádka, det. 2004 [p, orange label]'.

ParatyPe (甲): ‘MALAWI / Balaka env. / 5. - 6. i. 2002 [sic!] / J. Bezděk, lgt. [p, yellow label] // ex coll. L. Hromádka / National Museum / Prague, Czech Republic [p] // PARATYPUS / PHILONTHUS (s. str.) / pan sp.n. \& / L. Hromádka, det. 2004 [p, orange label]'.

PARATYPE (†): 'MALAWI S Masenjere / $80 \mathrm{~km}$ S of Blantyse / 21. -22., xii. 2001 / J. Bezděk, lgt. [p, yellow label] // ex coll. L. Hromádka / National Museum / Prague, Czech Republic [p] // PARATYPUS / PHILONTHUS (s. str.) / pan sp.n. + / L. Hromádka, det. 2004 [p, orange label]'.

ParatyPe (P): 'MALAWI S Jall env. / 30 km SE of Zomba / 26.-27., xii. 2001 / J. Bezděk, lgt. [p, yellow label] // ex coll. L. Hromádka / National Museum/Prague, Czech Republic [p] // PARATYPUS / PHILONTHUS (s. str.) / pan sp.n. + / L. Hromádka, det. 2004 [p, orange label]'.

PARATYPES ( 3 đô): 'MALAWI S Jall env. / $30 \mathrm{~km}$ SE of Zomba/ 26.-27., xii. 2001 / J. Bezděk, lgt. [p, yellow label] // ex coll. L. Hromádka / National Museum/Prague, Czech Republic [p] // PARATYPUS / PHILONTHUS (s. str.) / pan sp.n. ô / L. Hromádka, det. 2004 [p, orange label]'.

ParatyPe (đ): 'MALAWI centr. / Kasungu env. / 27. xii. 2001 / J. Bezděk, lgt. [p, yellow label] // ex coll. L. Hromádka / National Museum / Prague, Czech Republic [p] // PARATYPUS / PHILONTHUS (s. str.) / pan sp.n. § / L. Hromádka, det. 2004 [p, orange label]'.

Paratype (†): 'MALAWI centr. / Kasungu env. / 27. xii. 2001 / J. Bezděk, lgt. [p, yellow label] // ex coll. L. Hromádka / National Museum / Prague, Czech Republic [p] // PARATYPUS / PHILONTHUS (s. str.) / pan sp.n. + / L. Hromádka, det. 2004 [p, orange label]’.

ParatyPes (4 $\bigcirc$ ): ‘ $\circ$ [p, orange label] // MALAWI centr. / Kasungu env. /27. xii. 2001 / J. Bezděk, lgt. [p, yellow label] // ex coll. L. Hromádka / National Museum / Prague, Czech Republic [p] // PARATYPUS / PHILONTHUS (s. str.) / pan sp.n. + / L. Hromádka, det. 2004 [p, orange label]'.

Paratype ( + ): ‘ $q$ [p, orange label] // MALAWI c. Kahingina / Forest Reserve, $40 \mathrm{~km} / \mathrm{N}$ of Kasungu 29. xii. 01 / J. Bezděk, lgt. [p, yellow label] // ex coll. L. Hromádka / National Museum / Prague, Czech Republic [p] // PARATYPUS / PHILONTHUS (s. str.) / pan sp.n. † L. Hromádka, det. 2004 [p, orange label]'

ParatyPes ( 4 \% $)$ : 'MALAWI c. Kahingina / Forest Reserve, $40 \mathrm{~km} /$ $\mathrm{N}$ of Kasungu 29. xii. 01 / J. Bezděk, lgt. [p, yellow label] // ex coll. 
L. Hromádka / National Museum / Prague, Czech Republic [p] // PARATY PUS / PHILONTHUS (s. str.) / pan sp.n. + / L. Hromádka, det. 2004 [p, orange label]'.

Paratype ( $\hat{\partial}$, dissected genitalia on a separate plastic card): 'MALAWI c. Kahingina / Forest Reserve, $40 \mathrm{~km}$ / N of Kasungu 29. xii. 01 / J. Bezděk, lgt. [p, yellow label] // ex coll. L. Hromádka / National Museum / Prague, Czech Republic [p] // PARATYPUS / PHILONTHUS (s. str.) / pan sp.n. J / L. Hromádka, det. 2004 [p, orange label]'.

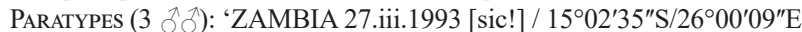
/ Kafue NP, Chunga / Camp, lux. leg. M. Uhlig [p, blue label] // ex coll. L. Hromádka / National Museum / Prague, Czech Republic [p] // PARATYPUS / PHILONTHUS (s. str.) / pan sp.n. §̋ / L. Hromádka, det. 2004 [p, orange label]'.

Paratype (ð): ‘ZIMBABWE 28.xi.-1.xii / 20³3'S/28³0’E / Matopos NP / lux, leg. M. Uhlig, 1993 [p, blue label] // ex coll. L. Hromádka/ National Museum / Prague, Czech Republic [p] // PARATYPUS / PHILONTHUS (s. str.) / pan sp.n. §/ L. Hromádka, det. 2004 [p, orange label]'.

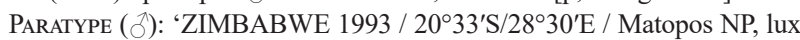
/ leg. M. Uhlig, 28.xi.-1.xii [p, blue label] // ex coll. L. Hromádka / National Museum / Prague, Czech Republic [p] // PARATYPUS / PHILONTHUS (s. str.) / pan sp.n. §ै / L. Hromádka, det. 2004 [p, orange label]'

Current status. Valid species.

Philonthus pandion Hromádka, 2013

Philonthus pandion Hromádka, 2013e: 231, Figs 78-81.

The holotype is deposited in NMPC (general collection):

Holotype (đ): 'LIBERIA/Mt. Nimba / Grassfield / 16.-25.ix. 1979 [p] // ex coll. L. Hromádka / National Museum / Prague, Czech Republic [p] // HOLOTYPE / PHILONTHUS s. str. / pandion sp.nov. / Hromádka det. 2009. [p, orange label]'.

Current status. Valid species.

\section{Philonthus pantodon Hromádka, 2010}

Philonthus pantodon Hromádka, 2010d: 108, Figs 27-30.

The holotype is deposited in NMPC (general collection): Holotype (ð): 'SUDAN mer. / Shambe / 15. 10. 1966 / Dr. P. Štys leg. [p, green label] // ex coll. L. Hromádka / National Museum / Prague, Czech Republic [p] // HOLOTYPUS / Philonthus (s. str.) / pantodon sp. nov. / Hromádka, det. 2009. [p, red label]'.

Current status. Valid species.

\section{Philonthus papio Hromádka, 2008}

Philonthus (s. str.) papio Hromádka, 2008c: 220, Figs 29-33.

Fifteen paratypes are deposited in NMPC (ex coll. L. Hromádka):

PARATYPe (ð): ‘ đ [p, pink label] // Rep.Guinea, / Sérédoux,lux [sic!] / 7.-8.4.1975 / leg.Zott [p] // ex coll. L. Hromádka / National Museum / Prague, Czech Republic [p] // PARATY PUS / PHILONTHUS (s.str.) / papio sp. n. / L. Hromádka, det. 2003 [p, yellow label]'

Paratypes (4 unsexed specimens): 'Rep.Guinea, / Sérédoux,lux [sic!] / 7.-8.4.1975 / leg.Zott [p] // ex coll. L. Hromádka / National Museum / Prague, Czech Republic [p] PARAT Y PUS / PHILONTHUS (s.str.) / papio sp. n. / L. Hromádka, det. 2003 [p, yellow label]'

ParatyPes (2 unsexed specimens): 'Guinea, / Seredou, lux / 4.5.1975 [sic!] / leg. Zott [p] // ex coll. L. Hromádka / National Museum / Prague, Czech Republic [p] // PARATYPUS / PHILONTHUS (s.str.) / papio sp. n. / L. Hromádka, det. 2003 [p, yellow label]'.

Paratypes (1 $\hat{\delta}, 1$ unsexed specimen): 'Rep.Guinea, / Seredou, lux / 5.IV.1975 [sic!] / leg.A.Zott [p] // ex coll. L. Hromádka / National Museum / Prague, Czech Republic [p] // PARATY PUS / Philonthus (s.str.) / papio sp. n. / L. Hromádka, det. 2001 [p, red label]'.

Paratype ( $\left.{ }^{\circ}\right)$ : 'Guinea, / Sérédou / 5.iv.1975 [sic! ] lux, leg. A. Zott [p, blue label] // ex coll. L. Hromádka / National Museum / Prague, Czech Republic [p] // PARATYPUS / PHILONTHUS (s.str.) / papio sp. n. / L. Hromádka, det. 2003 [p, yellow label]'.

PARATYPe (§): 'Guinea,Seredou / 7.-8.4.1975,lux / leg.Zott [p] // ex coll. L. Hromádka / National Museum / Prague, Czech Republic [p] // PARATYPUS / PHILONTHUS (s.str.) / papio sp. n. / L. Hromádka, det. 2003 [p, yellow label]'

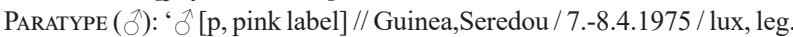
Zott [p] // ex coll. L. Hromádka / National Museum / Prague, Czech Republic [p] // PARATYPUS / PHILONTHUS (s.str.) / papio sp. n. / L. Hromádka, det. 2003 [p, yellow label]'.

PARATYPE ( $ठ$ ): ‘ $\delta$ [p, red label] // Rep. GUINEA/Seredou, lux / 5.IV.1975 [sic!] / leg.A.Zott [p] // ex coll. L. Hromádka / National Museum / Prague, Czech Republic [p] // PARATYPUS / Philonthus (s.str.) / papio sp. n. / L. Hromádka, det. 2001 [p, red label]'.

Paratype ( $)$ : ' $\delta$ [p, red label] // Rep. GUINEA / Sèrèdou, lux / 4.Apr.1975 [sic!] / leg.A.Zott [p] // ex coll. L. Hromádka / National Museum / Prague, Czech Republic [p] // PARATY PUS / PHILONTHUS (s.str.) / papio sp. n. ふै/ L. Hromádka, det. 2006 [p, red label]'

Paratype $(q$, dissected genitalia on a separate plastic card $):$ ' $q[\mathrm{p}$, orange label] // Republik Guinea / Sérédou, lux [p] II R / 3. Apr. [hw] 19 [p] 75 [hw] [sic!] / leg.Dr.A.Zott [p] // ex coll. L. Hromádka / National Museum / Prague, Czech Republic [p] // PARATYPUS / PHILONTHUS (s.str.) / papio sp. n. o / L. Hromádka, det. 2006 [p, orange label]'.

Current status. Valid species.

Philonthus papyrocranus Hromádka, 2013

Philonthus papyrocranus Hromádka, 2013e: 232, Figs 82-84.

The holotype and two paratypes are deposited in NMPC (general collection and ex coll. L. Hromádka):

Holotype (ð): 'ETHIOPIA: Bale / 8km. W. of Dinshu / 0706 N. 3944 E / 3.050m. xii. 1971 [p] // ex coll. L. Hromádka / National Museum / Prague, Czech Republic [p] // HOLOTYPE / PHILONTHUS (s. str.) / papyrocranus sp.nov. / Hromádka det., 2010. [p, orange label]'. Paratypes (2 A) : 'ETHIOPIA: Bale / 8km. W. of Dinshu / 0706 N. 3944 E. / 3.050m. xii. 1971 [p] // ex coll. L. Hromádka / National Museum / Prague, Czech Republic [p] // PARATYPE / PHILONTHUS (s. str.) / papyrocranus sp.nov. / Hromádka det., 2010. [p, orange label]'.

Current status. Valid species.

Philonthus pareutropicus Hromádka, 2013

Philonthus pareutropicus Hromádka, 2013e: 233, Figs 85-88.

The holotype and three paratypes are deposited in NMPC (general collection and ex coll. L. Hromádka):

HoLotype ( $\precsim$ ): 'Rep. South Africa / Northern Prov. Camp / David, 5km S. Ofoolaco / 475m 17.-24.i.2002 / leg. S. Murzin [p, green label] // ex coll. L. Hromádka / National Museum / Prague, Czech Republic [p] // HOLOTYPUS / PHILONTHUS (s. str.) / pareutropicus (sp. nov.) / Hromádka, det., 2010. [p, orange label]'.

ParatyPes ( 2 9 , 1 unsexed specimen): 'Rep. South Africa / Northern Prov. Camp / David, 5km S. Ofoolaco / 475m 17.-24.i.2002 / leg. S. Murzin [p, green label] // ex coll. L. Hromádka / National Museum / Prague, Czech Republic [p] // PARATY PUS / PHILONTHUS (s. str.) / pareutropicus (sp.nov.) / Hromádka, det., 2010. [p, orange label]'.

Current status. Valid species.

Philonthus pedetes Hromádka, 2013

Philonthus pedetes Hromádka, 2013e: 234, Figs 99-102.

The holotype and one paratype are deposited in NMPC (general collection and ex coll. L. Hromádka):

Holotype ( $)$ ) ' $\delta$ [p, green label] // Afrique or. allemande / KILIMANDJARO / VERSANT SUD·EST / ALLUAUD \& JEANNEL 
[p] // LISIÉRE SUPÉR. DES FORÉTS / BISMARC-HÜGEL / 2700-2800 m / Avril 1912 St. 71 [p] // ex coll. L. Hromádka / National Museum / Prague, Czech Republic [p] // HOLOTYPUS / PHILONTHUS (s. str.) / pedetes spec. nov. / Hromádka det. 2009. [p, orange label]'.

PARATYPE ( $($ ): 'PRAIRIES ALPINES / BISMARC-HÜGEL / 2600-2800' / Mars-Avril 1912 St. 70 [p] // Afrique or: allemande / KILIMANDJARO / VERSANT SUD·EST / ALLUAUD \& JEANNEL [p] // ex coll. L. Hromádka / National Museum / Prague, Czech Republic [p] // PARATYPUS / PHILONTHUS (s. str.) / pedetes sp. nov. / Hromádka det. 2009. [p, orange label]'.

Current status. Valid species.

\section{Philonthus pelecanus pelecanus Hromádka, 2010}

Philonthus pelecanus pelecanus Hromádka, 2010e: 42, Figs 111-115.

Two paratypes are deposited in NMPC (ex coll. L. Hromádka):

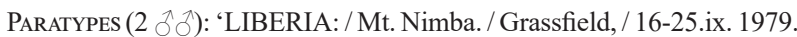
[p] // I. Hanski. / B.M. 1980-85. [p] // lowland / forest 500m. [p] // In human / faeces [p] // ex coll. L. Hromádka / National Museum / Prague, Czech Republic [p] // PARATYPUS / PHILONTHUS (s. str.) / pelecanus sp. n. / Hromádka, det. 2006. [p, red label]'.

Current status. Valid species.

Philonthus phacochoerus Hromádka, 2009

Philonthus phacochoerus Hromádka, 2009a: 677, Figs 20-26.

Two paratypes are deposited in NMPC (ex coll. L. Hromádka):

PARATYPe ( $\delta$, dissected genitalia mounted on a separate plastic card): ' $1 \mathrm{p}$, green label] // LIBERIA: / Mt. Nimba. / Grassfield, / 16-25. ix. 1979. [p] // lowland / forest 500m. [p] // In human / faeces [p] // I. Hanski. / B.M 1980-85. [p] // ex coll. L. Hromádka / National Museum / Prague, Czech Republic [p] // PARATYPUS / PHILONTHUS (s. str.) / phacochoerus sp. nov. / Hromádka, det. 2006. [p, orange label]'

Paratype ( $)$ ): ' 0 [p, green label] // LIBERIA: / Mt. Nimba. / Grassfield, / 16-25.ix. 1979. [p] // lowland / forest 500m. [p] // In human / faeces [p] // I. Hanski. / B.M 1980-85. [p] // ex coll. L. Hromádka / National Museum / Prague, Czech Republic [p] // PARATYPUS / PHILONTHUS (s. str.) / phacochoerus sp. nov. / Hromádka, det 2006. [p, orange label]'.

Current status. Valid species.

Philonthus phaon Smetana, 1995

Philonthus phaon Smetana, 1995: 167, Figs 186-190, 192, 193.

One paratype is deposited in NMPC (ex coll. L. Hromádka):

PARATYPe (unsexed specimen): 'ARIZ.Cochise Co.Chi- / ric.Mts.Rustler Park / 8400’24.VII.69 / A.Smetana [p] // ex coll. L. Hromádka / National Museum / Prague, Czech Republic [p] // PARATYPE / Philonthus / phaon / A. Smetana 1993 / CNC No.21951 [p, yellow label]'.

Current status. Valid species, see Herman (2001b).

\section{Philonthus philetarius Hromádka, 2014}

Philonthus philetarius Hromádka, 2014d: 690, Figs 7-9.

The holotype is deposited in NMPC (general collection):

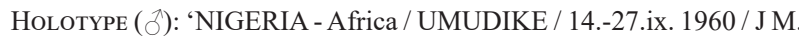
Gregory [p] // ex coll. L. Hromádka / National Museum / Prague, Czech Republic [p] // HOLOTYPUS / PHILONTHUS (s. str.) / philetarius nov.sp. / Hromádka, det., 2013. [p, red label]'.

Current status. Valid species.
Philonthus platalea Hromádka, 2010

Philonthus platalea Hromádka, 2010e: 43, Figs 116-122.

Five paratypes are deposited in NMPC (ex coll. L. Hromádka):

ParatyPes ( 1 o, 3 unsexed specimens): 'MUS. ROY.AFR.CENTR. / Yangambi (Stan.) / VII.1960 / J. Decelle [p] // ex coll. L. Hromádka / National Museum / Prague, Czech Republic [p] // PARATY PUS / PHILONTHUS / sg. Pseudophilonthus / platalea sp. nov. / Hromádka, det. 2006 [p, orange label]'.

ParatyPe ( $($ ): 'Belgyan Congo: / Oriental, / Yangambi [p] / 4-VII-1960 [hw] // Coll. J. Decelle / Field No. [p] 296 [hw] // ex termite / nest \#295[hw] // ex coll. L. Hromádka / National Museum/ Prague, Czech Republic [p] // PARATYPUS / PHILONTHUS / sg. Pseudophilonthus / platalea sp. nov. / Hromádka, det. 2006 [p, orange label]'.

Current status. Valid species.

Philonthus ploceus Hromádka, 2013

Philonthus ploceus Hromádka, 2013d: 447, Figs 144-147.

The holotype is deposited in NMPC (general collection): HoLOtYPe $\left({ }^{\precsim}\right)$ : 'ZIMBABWE / loc. Kutsaga / near Harare airport / 18.vi.1997 [sic!] W. Rossi leg. [p, yellow label] // ex coll. L. Hromádka / National Museum / Prague, Czech Republic [p] // HOLOTYPUS / PHILONTHUS (s. str.) / plocelus [sic!] sp. nov. / Hromádka, det., 2010 [p, orange label]'

Current status. Valid species.

Comments. Botswana is mentioned in the original description as the country of origin of the species; however, 'Kutsaga near Harare' is in Zimbabwe, as correctly written on the locality label.

Philonthus plotosus Hromádka, 2012

Philonthus plotosus Hromádka, 2012e: 186, Figs 25-28.

The holotype is deposited in NMPC (general collection): HoLOTYPE ('): 'Afrique or allemande / KILIMANDJARO / VERSANT SUD-EST [sic!] / ALLUAUD \& JEANNEL [sic!] [p] // ex coll. L. Hromádka / National Museum / Prague, Czech Republic [p] // HOLOTY PUS / PHILONTHUS (s. str.) / plotosus sp. nov. / Hromádka, det. 2010. [p, orange label]'.

Current status. Valid species.

Philonthus polemaetus Hromádka, 2011

Philonthus polemaetus Hromádka, 2011a: 201, Figs 48-50.

The holotype is deposited in NMPC (ex coll. L. Hromádka): HoLotype (ð̋): ‘TANZANIA / Mwanza / 11.x. 1969 / Ardö leg. [p] // ex coll. L. Hromádka / National Museum / Prague, Czech Republic [p] // HOLOTYPUS / PHILONTHUS (s. str.) / polemaetus spec. nov. / Hromádka, det., 2011. [p, orange label]'.

Current status. Valid species.

Philonthus polihierax Hromádka, 2014

Philonthus polihierax Hromádka, 2014j: 420, Figs 33-35.

The holotype is deposited in NMPC (general collection): HolotyPe ( $\left(^{\jmath}\right)$ : 'NAMIBIA - S / Hardap, 20km N / Mariental / 27. 30.03.1994 / ARNDT \& GRÖGER [p] // ex coll. L. Hromádka / National Museum / Prague, Czech Republic [p] // HOLOTYPUS / PHILONTHUS (s. str.) / polihierax sp.nov. / Hromádka, det., 2013. [p, red label]'.

Current status. Valid species. 
Philonthus princeps Bernhauer, 1902

Philonthus princeps Bernhauer, 1902: 56.

One syntype is deposited in NMPC (general collection): SynTYPe (§̊): 'AulieAta / SyrDaria [p] // COTYPUS [p, red label] // Rabigus / princeps / Bernhauer, 1902 / Hromádka, det 99. [p]’.

Current status. Rabigus princeps (Bernhauer, 1902), see SCHÜLKe \& SMETANA (2015).

\section{Philonthus profelis Hromádka, 2010}

Philonthus profelis Hromádka, 2010e: 46, Figs 123-126.

The holotype is deposited in NMPC (general collection): HolotyPe ( $\left.\jmath^{\jmath}\right)$ : ‘CONGO / Uele: Pawa / 1932 / Dr. A. Dubois [p, green label] // ex coll. L. Hromádka / National Museum / Prague, Czech Republic [p] // HOLOTYPUS / PHILONTHUS\{Pseudophi.) [sic!] / profelis sp.nov. / Hromádka, det. 2007 [p, red label]'.

Current status. Valid species.

Philonthus promerops Hromádka, 2014

Philonthus promerops Hromádka, 2014d: 691, Figs 12-14.

The holotype is deposited in NMPC (ex coll. L. Hromádka):

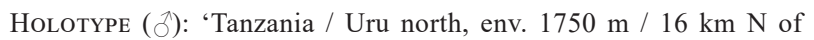
Moshi.v.2010 / Milan Kuboň lgt. [p] // ex coll. L. Hromádka / National Museum / Prague, Czech Republic [p] // HOLOTYPUS / PHILONTHUS (s. str.) / promerops sp. nov. / Hromádka, det., 2013 [p, red label]'.

Current status. Valid species.

\section{Philonthus pseudolus Smetana, 1995}

Philonthus pseudolus Smetana, 1995: 283, Figs 408-414.

Two paratypes are deposited in NMPC (ex coll. L. Hromádka):

ParatyPes ( $1 \delta 1$ + $)$ : 'ONT. Rondeau Pr. / Park end of / Marsh Trail 29. / V.85 A.Smetana [p] // ex coll. L. Hromádka / National Museum / Prague, Czech Republic [p] // PARATYPE / Philonthus / pseudolus / A. Smetana 1993 / CNC No.21956 [p, yellow label]'.

Current status. Philonthus pseudolodes Smetana, 1996, see Herman (2001b).

Comments. SMETANA (1996) proposed the new substitute name Philonthus pseudolodes Smetana, 1996 for Philonthus pseudolus Smetana, 1995, a junior primary homonym of Philonthus pseudolus Last, 1989.

\section{Philonthus psittacus Hromádka, 2008}

Philonthus (s. str.) psittacus Hromádka, 2008c: 221, Figs 34-38.

The holotype and four paratypes are deposited in NMPC (ex coll. L. Hromádka):

Holotype (ð): ‘ $\lesssim$ [p, green label] // ZAMBIA NC / 185 km S Mwinilunga / 6.12.2004 / Snížek \& Tichý [p] // ex coll. L. Hromádka / National Museum / Prague, Czech Republic [p] // HOLOTYPUS / PHILONTHUS (Philonthus) / psittacus sp. nov. / Hromádka, det. 2007 [p, orange label]'.

Paratypes (3 $\hat{\delta})$ : ' 0 [p, green label] // ZAMBIA NC / 185 km S Mwinilunga / 6.12.2004 / Snížek \& Tichý [p] // ex coll. L. Hromádka / National Museum / Prague, Czech Republic [p] // PARATYPUS / PHILONTHUS (Philonthus) / psittacus sp. nov. / Hromádka, det. 2007 [p, orange label]'.

Paratype ( + ): ‘ $q$ [p, orange label] // ZAMBIA NC / $185 \mathrm{~km} \mathrm{~S} \mathrm{Mwin-}$ ilunga / 6.12.2004 / Snížek \& Tichý [p] // ex coll. L. Hromádka /
National Museum / Prague, Czech Republic [p] // PARATYPUS / PHILONTHUS (Philonthus) / psittacus sp. nov. / Hromádka, det. 2007 [p, orange label]'.

Current status. Valid species.

\section{Philonthus ptilopsis Hromádka, 2014}

Philonthus ptilopsis Hromádka, 2014j: 424, Figs 39-42.

The holotype is deposited in NMPC (general collection): Holotype (đ) : 'TANZANIA / Mwanza / 11.x. 1969 / Ardö leg. [p] // ex coll. L. Hromádka / National Museum / Prague, Czech Republic [p] // HOLOTYPUS / PHILONTHUS (s. str.) / ptilopsis sp. nov. / Hromádka, det., 2013. [p, red label]’.

Current status. Valid species.

Philonthus (Rabigus) purkynei Smetana, 1963

Philonthus (Rabigus) purkyněi Smetana, 1963: 297, Fig. 1.

The holotype is deposited in NMPC (general collection): Holotype (đ): 'KaraKorum [hw] / Mongolia, V-VI 59/ C. Purkyně lgt. [p] // HOLOTYPUS [p] / Philonthus / purkyněi m. / Smetana det. [hw, red label] // Philonthus / purkyněi m. [hw] / Smetana det. 19 [p] 60 [hw]'.

Current status. Rabigus purkynei (Smetana, 1963), see SCHÜLKE \& SMETANA (2015).

Philonthus python Hromádka, 2010

Philonthus python Hromádka, 2010e: 47, Figs 127-128.

The holotype is deposited in NMPC (general collection): Holotype ( 9 ): ‘Tafa / 21.IV. 1959 / V.F.E [hw] // ex coll. L. Hromádka / National Museum / Prague, Czech Republic [p] // HOLOTY PUS / PHILONTHUS / sg. Pseudophilonthus / python sp. nov. / Hromádka,det.2006. [p, orange label]'.

Current status. Valid species.

Philonthus pytilia Hromádka, 2013

Philonthus pytilia Hromádka, 2013b: 10, Figs 21-22.

The holotype and one paratype are deposited in NMPC (general collection and ex coll. L. Hromádka):

Holotype ( ${ }^{\natural}$ ): 'TANGANYIKA: Mt.Meru / E slope, 5700 feet / Coll. Dr.J.SZUNYOGHY [p] // extracted from dung / of buffalo, / 26.I.1966 [sic!]. [p] // ex coll. L. Hromádka / National Museum / Prague, Czech Republic [p] // HOLOTYPUS / PHILONTHUS (s. str.) / pytilia sp. nov. / Hromádka, det. 2012. [p, red label]'.

ParatyPe (P): 'TANGANYIKA: Mt.Meru / E slope, 5700 feet / Coll. Dr.J.SZUNYOGHY [p] // extracted from dung/ of buffalo, / 26.I.1966 [sic!]. [p] // ex coll. L. Hromádka / National Museum / Prague, Czech Republic [p] // PARATYPUS / PHILONTHUS (s. str.) / pytilia sp. nov. / Hromádka, det., 2012. [p, red label]'.

Current status. Valid species.

\section{Philonthus quelea Hromádka, 2013}

Philonthus quelea Hromádka, 2013b: 11, Figs 23-25.

The holotype is deposited in NMPC (general collection):

HoLOTYPe ( $\left.\jmath^{\jmath}\right)$ : 'ZIMBABWE / loc. Kutsaga / near Harare airport / 18.vi.1997 W. Rossi leg. [p,yellow label] // ex coll. L. Hromádka / National Museum / Prague, Czech Republic [p] // HOLOTYPUS / PHILONTHUS (s. str.) / quelea sp.nov. / Hromádka, det. 2012. [p, red label]'.

Current status. Valid species. 
Philonthus ralumensis Bernhauer, 1915

Philonthus ralumensis Bernhauer, 1915: 188.

One syntype is deposited in NMPC (general collection):

SyntyPE (unsexed specimen): 'Neu-Britannien / Ralum / F.Dahl S. [p, violet label] // ralumensis / Brnh. Cotypus [hw]'.

Current status. Valid species, see Herman (2001b).

\section{Philonthus raphicerus Hromádka, 2013}

Philonthus raphicerus Hromádka, 2013e: 238, Figs 103-106.

Two paratypes are deposited in NMPC (ex coll. L. Hromádka):

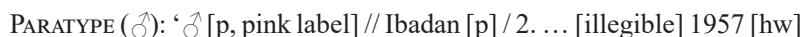
/ J L Gregory [p] // C.E. Tottenham / collection. / B.M. 1974-587. [p] // ex coll. L. Hromádka / National Museum / Prague, Czech Republic [p] // PARATYPUS / PHILONTHUS (s. str.) / raphicerus sp.nov. / Hromádka, det., 2009. [p, red label]'

PARATYPE ( ${ }^{\circledR}$ ): 'WEST AFRICA,Ghana / Northern Region, / Tamale / leg. Dr.S.Endrődi [p] // No. 52 / Lichtfalle/Quarz / / 30.VIII.1970. [p] // ex coll. L. Hromádka / National Museum / Prague, Czech Republic [p] // PARATYPUS / PHILONTHUS (s. str.) / raphicerus sp.nov. / Hromádka, det., 2009. [p, red label]'.

Current status. Valid species.

\section{Philonthus reitterianus Smetana, 1955}

Philonthus reitterianus Smetana, 1955: 208, Figs 5, 6.

One paratype is deposited in NMPC (general collection): Paratype ( 9 ): ‘[no locality data] // microcephalus [hw] // PARATYPE [p, red label] // Philonthus Curt. [p] / reitterianus / m. / paratype 54 $[\mathrm{hw}] /$ det. Smetana $19[\mathrm{p}]^{\prime}$.

Current status. Valid species, see SchüLKE \& SMETANA (2015).

Philonthus rhinopoma Hromádka, 2013

Philonthus rhinopoma Hromádka, 2013e: 239, Figs 107-109.

One paratype is deposited in NMPC (ex coll. L. Hromádka): Paratype (ふう): 'Oum Hadjer / Tschadgebiet / AEF.,lg.Franz [p] // ex coll. L. Hromádka / National Museum / Prague, Czech Republic [p] // PARATY PUS / PHILONTHUS (s. str.) / rhinopoma sp.nov. / Hromádka, det., 2012. [p, orange label]’.

Current status. Valid species.

Comments. HromádKa (2013e) erroneously assigned all three type specimens of $P$. rhinopoma to his private collection. However, the material originated from the collection of H. Franz in the Naturhistorisches Museum Wien (H. Schillhammer, pers. comm. 2020). Therefore, we have returned the holotype and one paratype to the museum in Vienna.

\section{Philonthus rotundicollis inopinatus Smetana, 1963}

Philonthus (Philonthus s. str.) rotundicollis inopinatus Smetana, 1963: 294.

The holotype and two paratypes (including the allotype) are deposited in NMPC (general collection):

Holotype (ठ): ‘Mongolia, V.-VI 59 / C. Purkyně lgt. [p] / Karakorum [hw] // HOLOTYPUS [p] / Philonthus / rotundicollis / s.inopinatus [hw] / Smetana det. [p, red label] // Philonthus Curt. [p]/ rotundicollis / s.inopinatus / m. 60 [hw] / det. Smetana 19 [p]'.
Paratype (+): 'Ulaanbaator / Mongolia, V- VI 59 / C. Purkyně lgt. [p] // ALLOTYPUS [p] / Philonthus / rotundicollis / s.inopinatus [hw]/ Smetana det. [p, red label]'.

Paratype (ठ)): 'Karakorum [hw] / Mongolia V-VI 59 / C. Purkyně lgt. [p] // ex coll. L. Hromádka / National Museum / Prague, Czech Republic [p] // PARATYPUS [p] / Philonthus / rotundicollis / ssp.inopina- / tus [hw] / Smetana det. [p, red label]'.

Current status. Junior subjective synonym of Philonthus rotundicollis (Ménétriés, 1832), see SCHILLHAMMER (2003) and SCHÜLKe \& SMETANa (2015).

Philonthus sabine Schillhammer, 2011

Philonthus sabine Schillhammer, 2011: 145, Fig 21.

One paratype is deposited in NMPC (ex coll. L. Hromádka): PARATYPE (unsexed specimen): 'CHINA-YUNNAN / 28.5.-9.6. 1994 DALI / lgt. E.Kučera [p] // ex coll. L. Hromádka / National Museum / Prague, Czech Republic [p] // PARATY PUS / Philonthus / sabine sp.n. / des. Schillhammer 2011 [p, orange label]'.

Current status. Valid species, see SchüLKE \& SMETANA (2015).

Philonthus sagittarius Hromádka, 2013

Philonthus sagittarius Hromádka, 2013d: 418, Figs 104-108.

One paratype is deposited in NMPC (ex coll. L. Hromádka):

PARATYPe ( $\overbrace{}^{\Uparrow})$ : 'ETHIOPIA:Bale / 8 km. W. of Dinshu / 0706 N.3944 E. / 3.050 m.xii.1971 [p] // R.O S.Clark / B.M.1973-450 [sic!] [p] // ex coll. L. Hromádka / National Museum / Prague, Czech Republic [p] // PARATY PUS / PHILONTHUS (s. str.) / sagittarius / Hromádka, det. 2009. [p, orange label]'.

Current status. Valid species.

\section{Philonthus scotopelia Hromádka, 2013}

Philonthus scotopelia Hromádka, 2013e: 243, Figs 115-117.

The holotype and two paratypes are deposited in NMPC (general collection and ex coll. L. Hromádka):

HоLотуPE (đ̊): 'RWANDA/ Nyakabuye / 15.-24.4.1984 / leg.H.Mühle [p] // ex coll. L. Hromádka / National Museum / Prague, Czech Republic [p] // HOLOTYPE / PHILONTHUS s. str. / scotopelia sp. nov. / Hromádka det. 2009. [p, orange label]'.

PARATYPES (2 ổ): 'RWANDA / Nyakabuya / 15.-24.iv.1984 / leg.,H. Mühle [p] // ex coll. L. Hromádka / National Museum / Prague, Czech Republic [p] // PARATYPUS / PHILONTHUS s. str. / scotopelia sp. nov. / Hromádka det., 2012. [p, orange label]'.

Current status. Valid species.

Philonthus siegwaldii Mannerheim, 1843

Philonthus Siegwaldii Mannerheim, 1843: 230.

One paralectotype is deposited in NMPC (general collection):

Paralectotype (unsexed specimen): 'Sitcha [p]// Holmberg [p]// Spec. typ. [p] / Phil. Siegwaldi / Mann. Cotype [hw, red label] // PARALECTOTYPE / PHILONTHUS / siegwaldii Mannerheim, 1843 / labelled by NMPC, 2019 [p, red label]'.

Current status. Bisnius siegwaldii (Mannerheim, 1843), see Smetana (1995) and Schülke \& SMEtana (2015).

Comments. MANnerheim (1852) described this species from an unknown number of specimens. The lectotype was designated by SMETANA (1995). Because there is no doubt 
about the authenticity of our specimen, we have labeled it as an additional paralectotype.

\section{Philonthus simonae Hromádka, 1992}

Philonthus [s.str.] simonae Hromádka, 1992: 99, Figs 6-8.

The holotype is deposited in NMPC (ex coll. L. Hromádka):

HоLOTYPe (ð): 'NEPAL-MANANG [sic!] / 15.10 .80 / A.ROUSEK / [reverse of the same label] 3500-4000 m/ PISANO ? [hw] // ex coll. L. Hromádka / National Museum / Prague, Czech Republic [p] // HOLOTYPUS / PHILONTHUS (s. str.) / simonae sp. nov. / Hromádka, det. 1992. [p, orange label]'.

Current status. Philonthus lubomiri Herman, 2001, see SChÜLKe \& SMetana (2015).

Comments. Herman (2001c) proposed the new substitute name Philonthus lubomiri Herman, 2001 for Philonthus simonae Hromádka, 1992, a junior primary homonym of Philonthus simonae Levasseur, 1962.

\section{Philonthus smithornis Hromádka, 2012}

Philonthus smithornis Hromádka, 2012d: 105, Figs 91-93.

The holotype is deposited in NMPC (general collection): HoLotyPe ( đ’): 'Rep. de Guinea / Wassako am Licht / 17.xi.1996. / Lange leg. [p] // ex coll. L. Hromádka / National Museum / Prague, Czech Republic [p] // HOLOTYPUS / PHILONTHUS (s. str.) / smithornis sp.nov. / Hromádka, det., 2011. [p, orange label]'.

Current status. Valid species.

\section{Philonthus stysi Hromádka, 1987}

Philonthus (s. str.) stysi Hromádka, 1987a: 21, Figs 1-3.

The holotype is deposited in NMPC (ex coll. L. Hromádka):

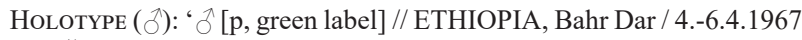
/ Štys/745 [p] // ex coll. L. Hromádka / National Museum / Prague, Czech Republic [p] // HOLOTYPUS / PHILONTHUS (s. str.) / stysi sp. n. § / L. Hromádka, det. 1987. [p, red label]’.

Current status. Valid species.

\section{Philonthus sula Hromádka, 2015}

Philonthus sula Hromádka, 2015b: 301, Figs 10-13.

Eight paratypes are deposited in NMPC (ex coll. L. Hromádka):

PARATyPes (6 えす): 'ETHIOPIA 30.04.2013 / TURMI 914 m alt. / N

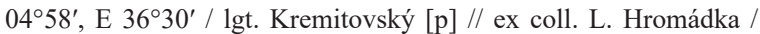
National Museum / Prague, Czech Republic [p] // PARATYPUS / PHILONTHUS (s. str.) / sula sp. nov. / Hromádka, det.,2015. [p, red label]'.

Paratypes ( $0^{\circ}+$ ): ‘ $\bigcirc$ [p] // ETHIOPIA 30.04.2013 / TURMI 914 m alt. / N 045ㅇ', E 36³0' / lgt. Kremitovský [p] // ex coll. L. Hromádka / National Museum / Prague, Czech Republic [p] // PARATYPUS / PHILONTHUS (s. str.) / sula sp. nov. / Hromádka, det.,2015. [p, red label]'.

Current status. Valid species.

Philonthus surikata Hromádka, 2012

Philonthus surikata Hromádka, 2012c: 161, Figs 60-62.

One paratype is deposited in NMPC (ex coll. L. Hromádka): PARAType (o): 'ETHIOPIA: / Simien, Arghine, / 11,500ft.or higher, / 24.xi.1952. [p] // Near torrent,in / peat soil; under / boulders or at / roots of plants [p] // ex coll. L. Hromádka / National Museum / Prague, Czech Republic [p] // PARATY PUS / PHILONTHUS (Philonthus) / suricata [sic!] sp.nov. / Hromádka, det. 2007 [p, red label]'.

Current status. Valid species.

Philonthus sylvisorex Hromádka, 2012

Philonthus sylvisorex Hromádka, 2012f: 71, Figs 43-46.

The holotype and one paratype are deposited in NMPC (general collection and ex coll. L. Hromádka):

Holotype (ð): 'BURUNDI / Kaninya / vii. 1940 / A.J. Bréda leg. [p, green label] // ex coll. L. Hromádka / National Museum / Prague, Czech Republic [p] // HOLOTYPUS / PHILONTHUS s. str. / sylvisorex sp.nov. / Hromádka, det., 2010. [p, red label]'.

Paratype (đ): ‘BURUNDI / Kaninya / vii. 1940 / A.J. Bréda leg. [p, green label] // ex coll. L. Hromádka / National Museum / Prague, Czech Republic [p] // PARATYPUS / PHILONTHUS s. str. / sylvisorex sp.nov. / Hromádka, det., 2010. [p, red label]'

Current status. Valid species.

Philonthus syncerus Hromádka, 2005

Philonthus (s.str.) syncerus Hromádka, 2005: 109, Figs 1-9.

Fourteen paratypes are deposited in NMPC (ex coll. L. Hromádka):

PARATYPE (ठ): ‘ $\delta$ [p, green label] // S.AFRICA:SKUKUZA / K.N.P. 29.i.1974 [sic!] / H.\& A Braack / B.M.1974-172 [p] // ex coll. L. Hromádka / National Museum / Prague, Czech Republic [p] // Desiderata / ex.NHM / 3.iii.2005 [p] // PARATYPUS / Philonthus (s. str.) / syncerus sp. n. / L. Hromádka, det. 2004. [p, orange label]'.

PARATYPE ( ): 'S.AFRICA:SKUKUZA / K.N.P. 27.i.1974 / H.\& A Braack / B.M.1974-172 [p] // ex coll. L. Hromádka / National Museum / Prague, Czech Republic [p] // Desiderata / ex.NHM / 3.iii.2005 [p] // PARATYPUS / Philonthus (s. str.) / syncerus sp. n. / L. Hromádka, det. 2004. [p, orange label]'.

PARATYPe (unsexed specimen): 'ANGOLA(A43) / 3 mls. N. / Santa Clara / 30.iii.-i.iv.1972 [p] // Southern / African Exp. / B.M.1972-I // ex coll. L. Hromádka / National Museum / Prague, Czech Republic [p] // Desiderata / ex.NHM / 3.iii.2005 [p] // PARATYPUS / Philonthus (s. str.) / syncerus sp. n. / L. Hromádka, det. 2004. [p, orange label]'.

PARATYPE (unsexed specimen): 'NAMIBIA 17.-18.xii.1993 / 19¹0'S/ $15^{\circ} 55^{\prime} \mathrm{E} /$ Etosha NP,Okaukuejo, / lux, leg. M. Uhlig [p, blue label] // ex coll. L. Hromádka / National Museum / Prague, Czech Republic [p] // PARATYPUS / Philonthus (s. str.) / syncerus sp. n. / L. Hromádka, det. 2004. [p, orange label]'.

PARATYPE (unsexed specimen): 'NAMIBIA 17.ii.1994 / 19²18'S / 14²2 'E [sic!] / Hobatere Lodge / leg. M. Uhlig [p, blue label] // ex coll. L. Hromádka / National Museum / Prague, Czech Republic [p] // PARATYPUS / PHILONTHUS (s. str.) / syncerus sp. n. / Hromádka, det. 2004. [p, orange label]'.

Paratype (ð): 'NAMIBIA 22.ii.1994 [sic!] / 18¹0'S/1357'E / Kaokoveld:10km SE / OPuvvo [sic!] ,lux,leg.M.Uhlig [p, blue label] // ex coll. L. Hromádka / National Museum / Prague, Czech Republic [p] // PARATYPUS / PHILONTHUS (s. str.) / syncerus / Hromádka, det. 2004 [p, orange label]'.

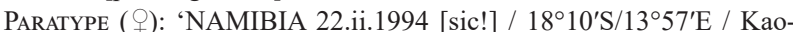
koveld:10km SE / OPuvvo [sic!], lux ,leg.M.Uhlig [p, blue label] // ex coll. L. Hromádka / National Museum / Prague, Czech Republic [p] // PARATYPUS / PHILONTHUS (s. str.) / syncerus sp. n. / Hromádka, det. 2004. [p, orange label]'.

Paratype $(\delta):$ : $\delta$, green label] // NAMIBIA 20.-21.ii.1994/ $17^{\circ} 00^{\prime} \mathrm{S} / 13^{\circ} 15^{\prime} \mathrm{E} /$ Kunene, Epupa / Falls, lux ,leg.M.Uhlig [p, blue label] // ex coll. L. Hromádka / National Museum / Prague, Czech Republic [p] // PARATYPUS / Philonthus (s. str.) / syncerus sp. n. / Hromádka, det. 2004 [p, orange label]'.

PARATYPe (unsexed specimen): 'BOTSWANA 8.-9.iii.1993 / 19²7'01"S/ $23^{\circ} 38^{\prime} 46^{\prime \prime} \mathrm{E} / 5 \mathrm{~km}$ NW San-ta- / Wani Safari Lodge / lux, leg. M. Uhlig 
[p, blue label] // ex coll. L. Hromádka / National Museum / Prague, Czech Republic [p] // PARATYPUS / PHILONTHUS (s. str.) / syncerus sp. n. / Hromádka, det. 2004 [p, orange label]'.

Paratypes (2 unsexed specimens): 'ZAMBIA 24.iii.1993 / 1306'03"S/ 31 47'32"E / South Luangwa NP / Mfuwe Crocodile Farm [sic!] / 450m,lux,leg. M.Uhlig [p, blue label] // ex coll. L. Hromádka / National Museum / Prague, Czech Republic [p] // PARATYPUS / PHILONTHUS (s. str.) / syncerus sp. n. / Hromádka, det. 2004 [p, orange label]'.

Paratype (unsexed specimen): 'ZAMBIA 21.iii.1993 / 1306'03"S /31 ${ }^{\circ} 47^{\prime} 32^{\prime \prime} \mathrm{E} /$ South Luangwa NP / Mfuwe Crocodile Farm [sic!] / 450m,lux,leg. M.Uhlig [p, blue label] // ex coll. L. Hromádka / National Museum / Prague, Czech Republic [p] // PARATYPUS / PHILONTHUS (s. str.) / syncerus sp. n. / L. Hromádka, det. 2004 [p, orange label]'.

Paratype (unsexed specimen): 'R.S.Africa 2.-3.ii.1994 / $27^{\circ} 36^{\prime} \mathrm{S} / 32^{\circ} 13^{\prime} \mathrm{E}$ / Natal: Mkuze NP: / lux, leg. M. Uhlig [p, blue label] // ex coll. L. Hromádka / National Museum / Prague, Czech Republic [p] // PARATYPUS / PHILONTHUS (s. str.) / syncerus sp. n. / Hromádka, det. 2004 [p, orange label]'.

PARATYPe (unsexed specimen): 'R.S.Africa 2.-3.ii.1994 / $27^{\circ} 36^{\prime} \mathrm{S} / 32^{\circ} 13^{\prime} \mathrm{E}$ / Natal: Mkuze NP: / lux, leg. M. Uhlig [p, blue label] // ex coll. L. Hromádka / National Museum / Prague, Czech Republic [p] // PARATYPUS / PHILONTHUS (s. str.) / syncerus sp. n. / L. Hromádka, det. 200 [p, red label]'.

Current status. Valid species.

Philonthus synodontis Hromádka, 2013

Philonthus synodontis Hromádka, 2013c: 200, Figs 30-32.

The holotype and two paratypes are deposited in NMPC (general collection and ex coll. L. Hromádka):

Holotype (ð): 'LIBERIA/ Mt. Nimba / Grassfield [sic!] / 16.-25.ix.1979 [p] // ex coll. L. Hromádka / National Museum / Prague, Czech Republic [p] // HOLOTYPUS / PHILONTHUS (s. str.) / synodontis spec.nov. / Hromádka, det. 2010. [p, orange label]'

PARATYPe (unsexed specimen): 'LIBERIA/ Mt. Nimba / Grassfield [sic!] / 16.-25.ix.1979 [p] // ex coll. L. Hromádka / National Museum / Prague, Czech Republic [p] // PARATYPUS / PHILONTHUS (s. str.) / synodontis spec.nov. / Hromádka, det. 2010. [p, orange label]'.

PARATyPe (unsexed specimen): 'SIERRA LEONE / Western Area / Base Picket Hill / 9.i. 1997 W. Rossi [p] // ex coll. L. Hromádka / National Museum / Prague, Czech Republic [p] // PARATYPUS / PHILONTHUS (s. str.) / synodontis spec.nov. / Hromádka, det. 2010. [p, orange label]'.

Current status. Valid species.

\section{Philonthus tachornis Hromádka, 2012}

Philonthus tachornis Hromádka, 2012c: 162, Figs 63-65.

The holotype is deposited in NMPC (ex coll. L. Hromádka):

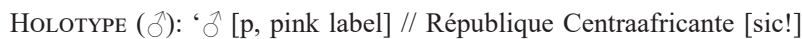
/ Bozo lumiere / 21. v. 1981 / leg. N. Degallier [p] // ex coll. L. Hromádka / National Museum / Prague, Czech Republic [p] // HOLOTYPUS / PHILONTHUS (s. str.) / tachornis / Hromádka, det., 2011. [p, orange label]'.

Current status. Valid species.

Philonthus tachymarptis Hromádka, 2013

Philonthus tachymarptis Hromádka, 2013e: 245, Figs 122-124.

The holotype is deposited in NMPC (general collection): HoLotyPe (ð) : 'CONGO BELGE / Libenge / 29. 4. 1948 / R.Cremer M. Neuman [p] // ex coll. L. Hromádka / National Museum / Prague, Czech Republic [p] // HOLOTYPUS / PHILONTHUS (s. str.) / tachymarptis sp. nov. / Hromádka, det., 2011. [p, red label]'.

Current status. Valid species
Philonthus tadarida Hromádka, 2013

Philonthus tadarida Hromádka, 2013d: 421, Figs 111-114.

The holotype is deposited in NMPC (ex coll. L. Hromádka): Holotype (ð̋): 'MALAWI S / Balaka / 19.xii. 2001 [sic!] / J. Bezděk, lgt. [p, yellow label] // ex coll. L. Hromádka / National Museum / Prague, Czech Republic [p] // HOLOTYPUS / PHILONTHUS (s. str.) / tadarida sp.nov. / Hromádka, det. 2009. [p, orange label]'.

Current status. Valid species.

Philonthus takanoi Hromádka, 2016

Philonthus takanoi Hromádka, 2016b: 29, Figs 12-14, 20, 23, 24.

Two paratypes are deposited in NMPC (ex coll. L. Hromádka):

Paratype (ठ): “TANZANiA 2224 / Mount Meru, Arusha N.P. S03 ${ }^{\circ}$ 14'54",E36 48'21" / 2-4.iv.12 Dung Pitfall / leg. Smith,R \& Takano,H [p] // BMNH\{E\} / 2012-92 / 1233195 [p] // ex coll. L. Hromádka / National Museum / Prague, Czech Republic [p] // PARATYPUS / PHILONTHUS (s. str.) / takanoi sp. nov. / Hromádka, det., 2013 [p, red label]'.

Paratype (ठ): 'TANZANiA 2224 / Mount Meru, Arusha N.P. S03 ${ }^{\circ} 4^{\prime} 54^{\prime \prime}, \mathrm{E} 36^{\circ} 48^{\prime} 21^{\prime \prime} /$ 2-4.iv.12 Dung Pitfall / leg. Smith,R \& Takano,H [p] // BMNH $\{$ E $\} /$ 2012-92 / 1233197 [p] // ex coll. L. Hromádka / National Museum / Prague, Czech Republic [p] // PARATYPUS / PHILONTHUS (s. str.) / takanoi sp. nov. / Hromádka, det., 2013 [p, red label]'.

Current status. Valid species.

Philonthus teleskopus Hromádka, 2013

Philonthus teleskopus Hromádka, 2013d: 424, Figs 122-124.

The holotype is deposited in NMPC (ex coll. L. Hromádka): HoLOTyPe (§̋): 'BURUNDI / Kaninya / vii. 1940 / A. J. Bréda [sic!] leg. [p, green label] // ex coll. L. Hromádka / National Museum / Prague, Czech Republic [p] // HOLOTYPUS / PHILONTHUS Stephens / telescopus [sic!] spec. nov. / Hromádka, det. 2010. [p, orange label]'.

Current status. Valid species.

Philonthus terathopius Hromádka, 2012

Philonthus terathopius Hromádka, 2012c: 163, Figs 66-68.

The holotype is deposited in NMPC (general collection):

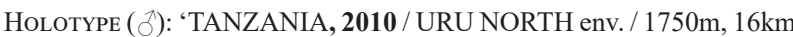
N of Moshi / May 10, Milan Kuboň lgt. [p] // ex coll. L. Hromádka / National Museum / Prague, Czech Republic [p] // HOLOTY PUS / PHILONTHUS (s. str.) / terathophius [sic!] sp. nov. / Hromádka, det. 2011. [p, orange label]'.

Current status. Valid species.

Comments. HromádKa (2012c: 163, 164) used three different original spellings, Philonthus terathopius, $P$. terathopis and $P$. terathopicus in the description of the species. As the specific epithet is derived from the Latin generic name of the African Bateleur Terathopius ecaudatus (Dudin, 1800), we fix the correct original spelling of the species as Philonthus terathopius according to Article 32.2.1 (ICZN 1999).

Philonthus terpsiphone Hromádka, 2012

Philonthus terpsiphone Hromádka, 2012d: 109, Figs 99-100.

The holotype and one paratype are deposited in NMPC (general collection and ex coll. L. Hromádka): 
Holotype ( đ): ‘Rep. de Guinea / Wassako am Licht / 17.xi..1996 / Lange, leg. [p] // ex coll. L. Hromádka / National Museum / Prague, Czech Republic [p] // HOLOTYPUS / PHILONTHUS (s. str.) / terpsiphone spec.nov / Hromádka, det., 2011. [p, orange label]'.

Paratype ( đ): 'Rep. de Guinea / Wassako am Licht / 17.xi.1996. / Lange leg. [p] // ex coll. L. Hromádka / National Museum / Prague, Czech Republic [p] // PARAT Y PUS / PHILONTHUS (s. str.) / terpsiphone sp. nov. / Hromádka det., 2011 [p, orange label]'.

Current status. Valid species.

Philonthus threskiornis Hromádka, 2013

Philonthus threskiornis Hromádka, 2013e: 245, Figs 125-128.

One paratype is deposited in NMPC (ex coll. L. Hromádka):

PARATYPe ( $\partial$, dissected genitalia on a separate plastic card): 'S.AFRICA. Mount.Sheba / E.Tvl.19.i.1974 / G.F.Bornemissza / B.M.1974-172 [p] // Pig / Dung [p] // ex coll. L. Hromádka / National Museum / Prague, Czech Republic [p] // PARATY PUS / PHILONTHUS (Philonthus) / threskiornis sp. nov. / Hromádka, det. 2006 [p, red label]'.

Current status. Valid species.

\section{Philonthus tockus Hromádka, 2012}

Philonthus tockus Hromádka, 2012c: 164, Figs 69-71.

Two paratypes are deposited in NMPC (ex coll. L. Hromádka):

Paratypes $(2 \hat{\delta})$ ): 'I.R.S.A.C.-MUS.CONGO / Kivu: T. Kalehe,Bitale / N. O. Kahusi, 1600 m [p] / (for.transit.) [hw] 29-VI-1951 / N. Leleup [p] // Récolté dans / 1'humus [p, green label] // ex coll. L. Hromádka / National Museum / Prague, Czech Republic [p] // PARATY PUS / PHILONTHUS (s. str.) / tockus sp. nov. / Hromádka, det. 2010 [p, orange label]'.

Current status. Valid species.

Philonthus torgos Hromádka, 2005

Philonthus (s.str.) torgos Hromádka, 2005: 111, Figs 18-23.

One paratype is deposited in NMPC (ex coll. L. Hromádka):

PARATyPe (unsexed specimen): 'ZIMBABWE 7.-9.xii.1993/ $18^{\circ} 17^{\prime} \mathrm{S} / 32^{\circ} 43^{\prime} \mathrm{E} /$ Nyanga NP: Rhodes / Dam, reed +fern / sievings, leg. M. Uhlig [p, blue label] // ex coll. L. Hromádka / National Museum / Prague, Czech Republic [p] // PARAT Y PUS / PHILONTHUS (s.str.) / torgos spec. nov. / Hromádka, det. 2001. [p, red label]'

Current status. Valid species, see HromÁDKa (2013d).

Philonthus tragelaphus Hromádka, 2009

Philonthus tragelaphus Hromádka, 2009a: 680, Figs 27-33.

Four paratypes are deposited in NMPC (ex coll. L. Hromádka):

Paratypes (2 $\left.\begin{array}{l}2 \\ 0\end{array}\right)$ : 'REP S AFR:Cape / Prov., Knysna, / Diepwalle, 12- / XII-1981 [p] // FMHD \#81-603 [sic!], / elephant / dung [sic!], S. Peck [p] // ex coll. L. Hromádka / National Museum / Prague, Czech Republic [p] // PARATY PUS / PHILONTHUS(Philonth.) / tragelaphus sp. nov. / Hromádka,det.2007 [p, red label]'.

ParatyPe (đ): 'REP.SO.AFR: Cape / Prov., Knysna, / Diepwalle, 23-XII1981 [p] // FMHD\#81-629, for. / litter, S. Peck, / berlese / FIELD MUSEUM [p] // ex coll. L. Hromádka / National Museum / Prague, Czech Republic [p] // PARATYPUS / PHILONTHUS(Philonth.) / tragelaphus sp. nov. / Hromádka,det.2007 [p, red label]'.

ParatyPe ( $\odot$ ): ‘ $q$ [p, orange label] // REP.S.AFR.: Cape / Prov., Knysna, / Diepwalle, 23.XII.1981 [p] // FMHD\#81-629, for. / litter, S. Peck / FIELD MUSEUM [p] // ex coll. L. Hromádka / National Museum / Prague, Czech Republic [p] // PARATYPUS / PHILONTHUS(Philonth.) / tragelaphus sp. nov. / Hromádka,det.2007 [p, red label]'.
Current status. Valid species.

Philonthus treron Hromádka, 2012

Philonthus treron Hromádka, 2012f: 74, Figs 54-56.

The holotype is deposited in NMPC (general collection): Holotype (ð): ‘TANZANIA / Mwanza / 11.x.1969 / Ardö leg. [p] // ex coll. L. Hromádka / National Museum / Prague, Czech Republic [p] // HOLOTYPUS / PHILONTHUS (s. str.) / treron sp. nov. / Hromádka, det. 2011. [p, red label]’.

Current status. Valid species.

Philonthus tringa Hromádka, 2013

Philonthus tringa Hromádka, 2013d: 451, Figs 154-155.

The holotype is deposited in NMPC (ex coll. L. Hromádka): HoLotype ( $($ ): 'République Centraafricante [sic!] / Bozo lumiere / 21. v. 1981 / leg. N. Degallier [p] // ex coll. L. Hromádka / National Museum / Prague, Czech Republic [p] // HOLOTYPUS / PHILONTHUS (s. str.) / tringa sp.nov. / Hromádka, det., 2013. [p, red label]'

Current status. Valid species.

Philonthus trionyx Hromádka, 2010

Philonthus trionyx Hromádka, 2010e: 59, Figs 161-165.

The holotype is deposited in NMPC (general collection): HoLotype ( đ̊): ‘Tanganika: Mpala, 780 m. / VIII-1953 / H. Bomans [p] // ex coll. L. Hromádka / National Museum / Prague, Czech Republic [p] // HOLOTYPUS / PHILONTHUS (s. str.) / trionyx sp.nov. / Hromádka, det., 2013. [p, yellow label]’.

Current status. Valid species.

\section{Philonthus triseriatus Bernhauer, 1928}

Philonthus triseriatus Bernhauer, 1928: 112.

One syntype is deposited in NMPC (general collection): SYNTYPE (unsexed specimen): '[one wing on a separate label] // MUSÉE DU CONGO [p] // Mongende / 21 [hw] / -IV-1921/. Schouteden [p] // CO TYPUS [p, red label] // triseriatus / Bernh. / Cotypus. [hw]'.

Current status. Philonthoblerius triseriatus (Bernhauer, 1928), see SchülKe \& SMETANA (2015).

\section{Philonthus trochocerus Hromádka, 2013}

Philonthus trochocerus Hromádka, 2013b: 13, Figs 26-28.

Two paratypes are deposited in NMPC (ex coll. L. Hromádka): ParatyPe (unsexed specimen): 'Tschadsee,AEF., / Insel Iba [sic!],lg. Franz [p] // ex coll. L. Hromádka / National Museum / Prague, Czech Republic [p] // PARATYPUS / PHILONTHUS (s. str.) / trochocerus sp. nov. / Hromádka, det., 2012. [p, red label]'.

ParatyPe (unsexed specimen): 'Tschadsee,Ile [p] / Iba [hw],AEF / lg.H. Franz [p] // ex coll. L. Hromádka / National Museum / Prague, Czech Republic [p] // PARATYPUS / PHILONTHUS (s. str.) / trochocerus sp. nov. / Hromádka, det., 2012. [p, red label]'.

Current status. Valid species.

\section{Philonthus turnix Hromádka, 2016}

Philonthus turnix Hromádka, 2016a: 104, Figs 16-19.

One paratype is deposited in NMPC (ex coll. L. Hromádka):

Paratype (đ): 'DŘC [sic!]; Tsingidi / 2.24. 15.6S12.58.11.0E / November 2013 / leg.L.J.Niemand [p] // ex coll. L. Hromádka / National Museum 
/ Prague, Czech Republic [p] // PARATYPUS / PHILONTHUS (s.str.) / turnix sp. nov. / Hromádka, det. 2015 [p, red label]'.

Current status. Valid species.

\section{Philonthus uhligi Hromádka, 2005}

Philonthus (s.str.) uhligi Hromádka, 2005: 112, Figs 29-31.

One paratype is deposited in NMPC (ex coll. L. Hromádka): PARATYPe (†): 'R.S.Africa 5.i.1999 [sic!] / Kruger NP: Skukuza / ex elephant dung / ex sample No. 9 / leg. C. Paetel [p, blue label] // ex coll. L. Hromádka / National Museum / Prague, Czech Republic [p] // PARATYPUS / PHILONTHUS (s. str.) / uhligi sp. n. / Hromádka,det. 2005 [p, red label]'.

Current status. Valid species.

\section{Philonthus umbrinoides Smetana, 1995}

Philonthus umbrinoides Smetana, 1995: 268, Figs 368-375.

Two paratypes are deposited in NMPC (ex coll. L. Hromádka):

Paratypes (1 $\delta$, 1 unsexed specimen): 'QUE:Parc Gati- / neau Lac Bourgeois 14.VI.79 / A.Smetana [p] // ex coll. L. Hromádka / National Museum / Prague, Czech Republic [p] // PARATYPE / Philonthus / umbrinoides / A. Smetana 1993 / CNC No.21953 [p, yellow label]'.

Current status. Valid species, see Herman (2001b).

\section{Philonthus upupa Hromádka, 2014}

Philonthus upupa Hromádka, 2014d: 692, Figs 18-20.

One paratype is deposited in NMPC (ex coll. L. Hromád$\mathrm{ka)}$ :

Paratype ( + ): 'LIBERIA: / Mt. Nimba. / Grassfield, / 16-25.ix. 1979. [p] // I. Hanski. / B.M 1980-85. [p] // lowland / forest 500m. [p] // In human / faeces [p] // ex coll. L. Hromádka / National Museum / Prague, Czech Republic [p] // PARATYPUS / PHILONTHUS (Philont.) / upupa sp. nov. / Hromádka, det. 2006 [p, orange label]'.

Current status. Valid species.

Philonthus uraeginthus Hromádka, 2012

Philonthus uraeginthus Hromádka, 2012f: 76, Figs 60-62.

The holotype and one paratype are deposited in NMPC (general collection and ex coll. L. Hromádka):

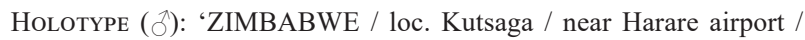
18.vi.1997 W. Rossi leg. [p, yellow label] // ex coll. L. Hromádka / National Museum / Prague, Czech Republic [p] // HOLOTYPE / PHILONTHUS (s. str.) / uraeginthus sp. n. / Hromádka, det., 2012. [p, orange label]'.

PARATYPE ( 3 ): 'ZIMBABWE / loc. Kutsaga / near Harare airport / 18.vi.1997 W. Rossi leg. [p, yellow label] // ex coll. L. Hromádka / National Museum / Prague, Czech Republic [p] // PARATY PUS / PHILONTHUS (s. str.) / uraeginthus sp. n. / Hromádka, det., 2012. [p, orange label]'.

Current status. Valid species.

\section{Philonthus veronikae Hromádka, 1992}

Philonthus [s.str.] veronikae Hromádka, 1992: 101, Figs 9-11.

The holotype is deposited in NMPC (ex coll. L. Hromádka):

Holotype (ठ): 'NEPAL-Khudi / 10.10.1980 / J.SEIFERT [hw] // ex coll. L. Hromádka / National Museum / Prague, Czech Republic [p] // HOLOTYPUS / PHILONTHUS (s. str.) / veronikae sp. nov. / Hromádka, det. 1992. [p, orange label]'.
Current status. Valid species, see SchüLKE \& SMETANA (2015).

\section{Philonthus vulpes Hromádka, 2012}

Philonthus vulpes Hromádka, 2012d: 112, Figs 88-90.

Three paratypes are deposited in NMPC (ex coll. L. Hromádka):

ParatyPe ( $\precsim$ ): 'RHODESIE du Nord / Ntondwa / 28. VIII. 1939 [sic!] / H. J. Bréda [p] // ex coll. L. Hromádka / National Museum / Prague, Czech Republic [p] // PARATYPUS / PHILONTHUS (s. str.) / vulpes sp. nov. / Hromádka det.2007. [p, red label]'.

ParatyPes (2 $\left.\delta^{\lambda}\right)$ : 'Kenya: [p] 17.VIII. [hw] / Treetops Hotel / Aberdare [p] 1960 [hw] / National Park [p] // Sifted from/ elephant manure [p] // Coll. D. H. \& / A. C. Kistner \& / R. Banfill / Field No. [p] 618 [hw] // ex coll. L. Hromádka / National Museum / Prague, Czech Republic [p] // PARATYPUS / PHILONTHUS(Spatulon.) / vulpes sp.nov. / Hromádka,det.2007 [p, red label]'.

Current status. Valid species.

\section{Philonthus xenopus Hromádka, 2011}

Philonthus xenopus Hromádka, 2011c: 185, Figs 84-87.

The holotype is deposited in NMPC (general collection): HoLOTYPe ( ð’): 'BURUNDI / Kaninya / vii. 1940 [sic!] / A. J. Bréda leg. [p, green label] // ex coll. L. Hromádka / National Museum / Prague, Czech Republic [p] // HOLOTYPE / PHILONTHUS (s. str.) / xenopus spec. nov. / Hromádka, det., 2010. [p, orange label]'.

Current status. Valid species.

\section{Philonthus xerus Hromádka, 2008}

Philonthus (s. str.) xerus Hromádka, 2008c: 229, Figs 54-58.

The holotype and four paratypes are deposited in NMPC (ex coll. L. Hromádka):

Holotype (đ): ' o [p, red label] // SUDAN 22.08.1966/ $21^{\circ} 48^{\prime} 00^{\prime \prime} \mathrm{N} 031^{\circ} 20^{\prime} 00^{\prime \prime} \mathrm{E} / \mathrm{Khartoum}(373) / \mathrm{Dr}$. P. Štys leg. [p, green label] // ex coll. L. Hromádka / National Museum / Prague, Czech Republic [p] // HOLOTYPUS / PHILONTHUS (Philon.) / xerus sp. nov. / Hromádka, det. 2006 [p, red label]'.

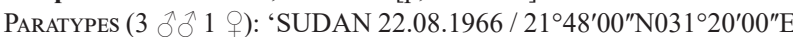
/ Khartoum (373) / Dr. P. Štys leg. [p, green label] // ex coll. L. Hromádka / National Museum / Prague, Czech Republic [p] // PARATYPUS / PHILONTHUS (Philon.) / xerus sp. nov. / Hromádka, det. 2006 [p, red label]’.

Current status. Valid species.

Philonthus zoothera Hromádka, 2013

Philonthus zoothera Hromádka, 2013b: 14, Figs 29-32.

One paratype is deposited in NMPC (ex coll. L. Hromádka):

Paratype (đ) : 'BluKwa / 12.1.29 [hw] / A.Collar [p] // ex coll. L. Hromád$\mathrm{ka} /$ National Museum/ Prague, Czech Republic [p] // PARATY PUS / PHILONTHUS (s.str.) / zoothera sp. nov. / Hromádka, det. 2009. [p, orange label]'.

Current status. Valid species.

Philonthus zosterops Hromádka, 2011

Philonthus zosterops Hromádka, 2011e: 194, Figs 52-56.

The holotype and one paratype are deposited in NMPC (ex coll. L. Hromádka):

HoLOTYPe (ð̊): ‘RHODESIE du Nord / Ntondwa / 28. VIII. 1939 / H. J. 
Bréda [p] // ex coll. L. Hromádka / National Museum / Prague, Czech Republic [p] // HOLOTYPUS / PHILONTHUS (s. str.) / zosterops sp. nov. / Hromádka, det., 2010. [p, orange label]'.

ParatyPe (đ): 'Rhodésie du Nord / Ntondwa / 28-VIII-1939 / H. J. Brédo [sic!] [p] // R. Mus. Hist. Nat. / Belg. I. G. 13.212 [p] // ex coll. L. Hromádka / National Museum / Prague, Czech Republic [p] // HOLOTY PUS / PHILONTHUS (s. str.) / zosterops sp. nov. / Hromádka, det., 2010. [p, orange label]'.

Current status. Philonthus zosterops Hromádka, 2011. Comments. HromÁDKa (2016a) proposed the new substitute name Philonthus mesophoyx Hromádka, 2016 for Philonthus zosterops Hromádka, 2011, supposedly a junior primary homonym of Philonthus zosterops Paulian, 1952. However, there is no such name in PaUlian (1952) and thus, $P$. mesophoy $x$ represents an unnecessary replacement name, here formally placed in synonymy with $P$. zosterops Hromádka, 2011.

\section{Philonthus zosterops Hromádka, 2013}

Philonthus zosterops Hromádka, 2013d: 443, Figs 136-137.

The holotype is deposited in NMPC (general collection): HoLOTYPe (P): 'ZIMBABWE / loc. Kutsaga / near Harare airport / 18.vi.1997 W.Rossi leg. [p, yellow label] // ex coll. L. Hromádka / National Museum / Prague, Czech Republic [p] // HOLOTYPUS / PHILONTHUS (s.str.) / zosterops sp. nov. / Hromádka, det. 2010. [p, orange label]'.

Current status. Philonthus acervulus Mikátová, Macháčková \& Hájek, nom. nov.

Comments. Philonthus zosterops Hromádka, 2013 is a junior primary homonym of Philonthus zosterops Hromádka, 2011. To remove the homonymy, we propose a new substitute name Philonthus acervulus nom. nov. for Philonthus zosterops Hromádka, 2013. The new name is a Latin translation of the name 'Hromádka'; it is a noun in nominative case.

\section{Pseudohesperus apsilus Hromádka, 2010}

Pseudohesperus apsilus Hromádka, 2010b: 497, Figs 1-5.

The holotype is deposited in NMPC (general collection): HoLOTYPE (đ): ‘UGANDA/Nakiwogo / Entebbe / XI.1961-IV.1962 [sic!] / A.S.Haddow [sic!] [hw] // ex coll. L. Hromádka / National Museum / Prague, Czech Republic [p] // HOLOTYPUS / Pseudohesperus Hayashi / apsilus sp. nov. / Hromádka det. 2010. [p, orange label]'.

Current status. Eccoptolonthus apsilus (Hromádka, 2010), see NeWTON (2015).

\section{Pseudohesperus tauraco Hromádka, 2010}

Pseudohesperus tauraco Hromádka, 2010b: 508, Figs 13-17.

Three paratypes are deposited in NMPC (ex coll. L. Hromádka):

ParatyPe (đ): ‘I.R.S.A.C.-MUS.CONGO / Kivu : T. Kalehe,Bitale / N. O. Kahusi, 1600 m (for transit) [hw] / 29-VI-1951 [p] / N. Leleup [p] // Récolté dans / l'humus [p] // ex coll. L. Hromádka / National Museum / Prague, Czech Republic [p] // PARATYPUS / Pseudohesperus Hayashi / tauraco sp. nov. / Hromádka det. 2010. [p, orange label]'

ParatyPe (ð): 'Massif Ruwenzori / Kyandolire, 1.750 m / Riv. Mulaku affl. / Kakalari (terreau) [p] // Congo Belge : P.N.A. / 16-x-1952 / P. Vanschuytbroeck \& / J. Kekenbosch 1302 [p] // ex coll. L. Hromádka / National Museum / Prague, Czech Republic [p] // PARATY PUS / Pseudohesperus Hayashi / tauraco sp. nov. / Hromádka, det., 2010. [p, orange label]'
PARATYPE (unsexed specimen): 'Massif Ruwenzori / Kalonge, $2.210 \mathrm{~m} /$ (terreau) [p] // Congo Belge : P.N.A. / 4-IX-1952 / P. Vanschuytbroeck \& / J. Kekenbosch 934 [p] // ex coll. L. Hromádka / National Museum / Prague, Czech Republic [p] // PARATY PUS / Pseudohesperus Hayashi / tauraco sp. nov. / Hromádka, det., 2010. [p, orange label]'.

Current status. Eccoptolonthus tauraco (Hromádka, 2010), see NewTon (2015).

Pseudohesperus terezae Hromádka, 2016

Pseudohesperus terezae Hromádka, 2016c: 376, Figs 10-13, 21.

The holotype is deposited in NMPC (general collection): HoLOTYPe ( $5 \mathrm{~km} \mathrm{~S}$ Ofcolaco [sic!] / 475 m, 17.-24. I. 2002 / leg S. Murzin [p, yellow label] // ex coll. L. Hromádka / National Museum / Prague, Czech Republic [p] // HOLOTYPUS / PSEUDOHESPERUS / terezae sp. nov. / Hromádka, det., 2015 [p, red label]’.

Current status. Eccoptolonthus terezae (Hromádka, 2016) comb. nov.

Comments. HromáDKa (2010b) proposed a new generic concept for Pseudohesperus Hayashi, 2008 that included Philonthus conradti Bernhauer, 1912 (the type species of Eccoptolonthus Bernhauer, 1912) which has priority over Pseudohesperus. Therefore, NewTON (2015) formally synonymised both genera and combined all hitherto known Pseudohesperus species with Eccoptolonthus. Following that, we propose here the new combination, Eccoptolonthus terezae (Hromádka, 2016) comb. nov., for Pseudohesperus terezae Hromádka, 2016.

\section{Pseudohesperus tyto Hromádka, 2010}

Pseudohesperus tyto Hromádka, 2010b: 509, Figs 23-27.

Two paratypes are deposited in NMPC (ex coll. L. Hromádka):

Paratype (ð): ‘Abessin, 1914 / Maraco 5. V. [hw] // ex coll. L. Hromádka / National Museum / Prague, Czech Republic [p] // PARATY PUS / Pseudohesperus Hayashi / tyto sp. nov. / Hromádka det. 2010. [p, orange label]'.

ParatyPe (ð’): 'CONGO BELGE / Libenge / 29. 4. 1948 / R.Cremer M. Neuman [p] // ex coll. L. Hromádka / National Museum / Prague, Czech Republic [p] // PARATYPUS / Pseudohesperus Hayashi / tyto sp. nov. / Hromádka det. 2010. [p, orange label]'.

Current status. Eccoptolonthus tyto (Hromádka, 2010), see NeWTON (2015).

\section{Pseudohesperus varanus Hromádka, 2010}

Pseudohesperus varanus Hromádka, 2010b: 511, Figs 28-32.

One paratype is deposited in NMPC (ex coll. L. Hromádka): PARATYPE (§)): 'I.R.S.A.C.-MUS.CONGO / Kibali-Ituri : Terr. Djugu, / Mt. Aboro, 2200 m. / N. Leleup / I-1954 [p] // ex coll. L. Hromádka / National Museum / Prague, Czech Republic [p] // PARATYPUS / PSEUDOHESPERUS / varanus sp. nov. / Hromádka, det., 2010. [p, orange label]'.

Current status. Eccoptolonthus varanus (Hromádka, 2010), see Newton (2015).

\section{Shaverdolena laosensis Schillhammer, 2011}

Shaverdolena laosensis Schillhammer, 2011: 148, Fig. 23.

The holotype is deposited in NMPC (general collection): 


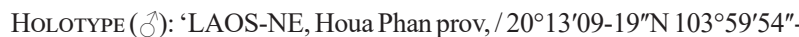
/ 104 00'03"E, 1480-1510m, / PHOU PANA Mt.,22.iv.-14.v. / 2008,Vít Kubáň leg. // HOLOTYPUS / Shaverdolena / laosensis sp.n. / des. Schillhammer 2011 [p, red label]'.

Current status. Valid species.

\section{Acknowledgements}

We are obliged to Alfred F. Newton (Field Museum of Natural History, Chicago, USA) and Harald Schillhammer (Naturhistorisches Museum Wien, Vienna, Austria) for valuable comments on the manuscript. The work was supported by the Ministry of Culture of the Czech Republic (DKRVO 2019-2023/5.I.b, National Museum, 00023272).

\section{References}

BATELKA J. \& HÁJEK J. 2015: Catalogue of type specimens of beetles (Coleoptera) deposited in the National Museum, Prague, Czech Republic. Meloidae and Ripiphoridae. Acta Entomologica Musei Nationalis Pragae 55: 825-858.

BERNHAUER M. 1902: Beitrag zur Staphylinidenfauna des palaearctischen Gebietes. Münchener Koleopterologische Zeitschrift 1: 54-62.

BERNHAUER M. 1910: Beitrag zur Kenntnis der Staphyliniden-Fauna von Zentralamerika. Verhandlungen der Kaiserlich-Königlichen Zoologisch-Botanischen Gesellschaft in Wien 60: 350-393.

BERNHAUER M. 1912: Zur Staphylinidenfauna von Südamerika. Entomologische Blätter 8: 167-179.

BERNHAUER M. 1914: Neue Staphyliniden der paläarktischen Fauna. Coleopterologische Rundschau 4: 65-68.

BERNHAUER M. 1915: Beitrag zur Staphylinidenfauna von Neu-Guinea. Deutsche Entomologische Zeitschrift 1915: 179-202.

BERNHAUER M. 1917: Beitrag zur Staphylinidenfauna des tropischen Afrika. Entomologische Blätter 13: 46-49.

BERNHAUER M. 1928. Zur Staphylinidenfauna des tropischen Afrika, insbesonders des Kongostaates. Wiener Entomologische Zeitung 45(4): 105-121.

BEZDĚČKOVÁ K., BEZDĚČKA P., MACEK J. \& MALENOVSKÝ I. 2017: Catalogue of type specimens of ants (Hymenoptera: Formicidae) deposited in Czech museums. Acta Entomologica Musei Nationalis Pragae 57: 295-308.

BEZDĚK A., BOUCHER S. \& HÁJEK J. 2017: Catalogue of type specimens of beetles (Coleoptera) deposited in the National Museum, Prague, Czech Republic. Scarabaeoidea: Lucanidae and Passalidae. Acta Entomologica Musei Nationalis Pragae 57: 279-293.

BEZDĚK A. \& HÁJEK J. 2009: Catalogue of type specimens of beetles (Coleoptera) deposited in the National Museum, Prague, Czech Republic. Scarabaeoidea: Bolboceratidae, Geotrupidae, Glaphyridae, Hybosoridae, Ochodaeidae and Trogidae. Acta Entomologica Musei Nationalis Pragae 49: 297-332.

BOHÁČ J. 1981: Results of the Czechoslovak-Iranian entomological expeditions to Iran. Coleoptera: Staphylinidae. Three new species of staphylinids from Iran. Acta Entomologica Musei Nationalis Pragae 40: $355-358$.

BOHÁČ J. \& HROMÁDKAL. 1977: Philonthus jureceki sp. n. from Bulgaria (Coleoptera, Staphylinidae). Acta Entomologica Bohemoslovaca 74: $283-285$.

BOHÁČ J. \& HROMÁDKAL. 1980: Philonthus jurecekianus nom. n. (Coleoptera, Staphylinidae). Acta Entomologica Bohemoslovaca 77: 430

CHANI-POSSE DE MAUS M. 2009: Revision of some types of Philonthus Stephens and Gabrius Stephens from southern South America (Coleoptera: Staphylinidae), with nomenclatural notes. Zootaxa 2034: 31-42.

CHANI-POSSE DE MAUS M., BRUNKE A. J., CHATZIMANOLIS S., SCHILLHAMMER H. \& SOLODOVNIKOV A. 2018: Phylogeny of the hyper-diverse rove beetle subtribe Philonthina with implications for classification of the tribe Staphylinini (Coleoptera: Staphylinidae). Cladistics 34: 1-40.

COIFFAIT H. 1974: Coléoptères staphylinides de la région paléarctique occidentale II. Sous famille Staphilininae, Tribus Philonthini et Sta- phylinini. Nouvelle Revue d'Entomologie, Supplément 4(4): 1-593.

DVOŘÁK R. 1957: A character useful in separating Cafius (sg. Bryonomus Csy.) seminitens Horn and canescens Makl. (Coleoptera: Staphylinidae). Entomological News 68: 17-18.

GEISER M. \& BARCLAY M. V. L. 2017: A replacement name for Philonthus colius Hromádka, 2016 (Coleoptera: Staphylinidae). Studies and Reports, Taxonomical Series 13: 61.

GEMMINGER M. \& HAROLD E. vON 1868: Catalogus Coleopterorum hucusque descriptorum synonymicus et systematicus. Tom. II. Dytiscidae, Gyrinidae, Hydrophilidae, Staphylinidae, Pselaphidae, Gnostidae, Paussidae, Scydmaenidae, Silphidae, Trichopterygidae, Scaphidiidae. E. H. Gummi, Monachii, pp. 425-752 + 6 .

HERMAN L. H. 2001a: Catalog of the Staphylinidae (Insecta: Coleoptera). 1758 to the end of the second millenium. I. Introduction, history, biographical sketches, and Omaliine group. Bulletin of the American Museum of Natural History 265: 1-650.

HERMAN L. H. 2001b: Catalog of the Staphylinidae (Insecta: Coleoptera). 1758 to the end of the second millenium. V. Staphylinine group (part 2) Staphylininae: Diochini, Maorothiini, Othiini, Platyprosopini, Staphylinini (Amblyopinina, Anisolinina, Hyptiomina, Philonthina). Bulletin of the American Museum of Natural History 265: vi + 2441-3020.

HERMAN L. H. 2001c: Nomenclatural changes in the Staphylinidae (Insecta: Coleoptera). Bulletin of the American Museum of Natural History 264: 1-83.

HROMÁDKAL. 1987a: Eine neue Philonthus-Art aus Äthiopien (Insecta, Coleoptera, Staphylinidae, Staphylininae). Reichenbachia 25: 21-22.

HROMÁDKA L. 1987b: Zwei neue Philonthus-Arten aus der Sowjetunion (Coleoptera, Staphylinidae). Entomologische Blätter 83: 147-150.

HROMÁDKA L. 1992: Vier neue Philonthus-Arten aus Nepal. Deutsche Entomologische Zeitschrift, Neue Folge 39: 97-102.

HROMÁDKA L. 2001: Zwei neue Staphyliniden-Arten aus Iran (Insecta: Coleoptera: Staphylinidae: Tachyporinae et Staphylininae). Reichenbachia 34: 137-141.

HROMÁDKA L. 2004: Two new Hesperus species (Coleoptera: Staphylinidae) from Tanzania and Nepal. Folia Heyrovskyana 12: 113-117.

HROMÁDKA L. 2005: New species of the genus Philonthus Stephens, 1829 from Aftrotropical region (Coleoptera, Staphylinidae, Philonthini). Coleoptera 9: 109-116.

HROMÁDKA L. 2006: A new species of Philonthus from Sierra Leone. Fragmenta Entomologica 38: 65-68.

HROMÁDKAL. 2007: Gabrius phelsuma sp. n. (Coleoptera: Philonthina) from Madagascar. Klapalekiana 43: 187-190.

HROMÁDKAL. 2008a: Revision of Afrotropical species of the Philonthus abyssinus species group (Coleoptera: Staphylinidae: Philonthina). Acta Entomologica Musei Nationalis Pragae 48: 37-50.

HROMÁDKAL. 2008b: Revision of Afrotropical species of the Philonthus peripateticus species group (Coleoptera: Staphylinidae: Philonthina). Acta Entomologica Musei Nationalis Pragae 48: 51-65.

HROMÁDKAL. 2008c: Revision of the Afrotropical species of Philonthus (s. str.) turbidus species-group (Coleoptera: Staphylinidae: Philonthina). Klapalekiana 44: 207-232.

HROMÁDKA L. 2009a: Five new Philonthus species (Coleoptera: Staphylinidae: Philonthina). Acta Entomologica Musei Nationalis Pragae 49: 671-682.

HROMÁDKA L. 2009b: Revision of the Afrotropical species of the genus Mentophilonthus (Coleoptera: Staphylinidae: Philonthina). Acta Entomologica Musei Nationalis Pragae 49: 119-160.

HROMÁDKA L. 2009c: Revision of the Afrotropical species of the Philonthus caffer species group (Coleoptera: Staphylinidae: Philonthina). Acta Entomologica Musei Nationalis Pragae 49: 161-190.

HROMÁDKA L. 2009d: Two new species of the genus Hesperus (Coleoptera: Staphylinidae: Philonthina) from the Afrotropical region. Klapalekiana 45: 67-72.

HROMÁDKA L. 2010a: A revision of the Aftrotropical species of the Philonthus arrowianus species group (Coleoptera: Staphylinidae: Philonthina). Acta Entomologica Musei Nationalis Pragae 50: 131-144.

HROMÁDKA L. 2010b: Revision of the Afrotropical species of the genus Pseudohesperus, with taxonomic notes on other Afrotropical species of the subtribe Philonthina (Coleoptera: Staphylinidae). Acta Entomologica Musei Nationalis Pragae 50: 495-516.

HROMÁDKAL. 2010c: Revision of Afrotropical species of the Philonthus 
aemulus species group (Coleoptera: Staphylinidae: Philonthina). Acta Societatis Zoologicae Bohemicae 73 [2009]: 27-51.

HROMÁDKAL. 2010d: Revision of Afrotropical species of the Philonthus quisquiliarius species group (Coleoptera: Staphylinidae: Philonthina). Studies and Reports, Taxonomical Series 6: 95-113.

HROMÁDKA L. 2010e: Revision of the Afrotropical species of the Philonthus (Philonthus) bicoloripennis species group (Coleoptera: Staphylinidae: Philonthina). Klapalekiana 46: 3-68.

HROMÁDKA L. 2010f: Revision of the Afrotropical species of the Philonthus marginipennis species group (Coleoptera: Staphylinidae: Philonthina). Acta Societatis Zoologicae Bohemicae 73 [2009]: 53-64.

HROMÁDKA L. 2011a: New species of genus Philonthus from the Afrotropical region (Coleoptera: Staphylinidae: Philonthina). Studies and Reports, Taxonomical Series 7: 189-206.

HROMÁDKA L. 2011b: Revision of Afrotropical species of the Philonthus cupreonitens species group (Coleoptera: Staphylinidae: Philonthina). Acta Societatis Zoologicae Bohemicae 75: 37-66.

HROMÁDKA L. 2011c: Revision of Afrotropical species of the Philonthus peliomerus species group (Coleoptera: Staphylinidae: Philonthina). Studies and Reports, Taxonomical Series 7: 151-188.

HROMÁDKA L. 2011d: Revision of the Afrotropical species of the genus Afrorabigus (Coleoptera: Staphylinidae: Philonthina). Journal of the National Museum (Prague), Natural History Series 180: 63-79.

HROMÁDKA L. 2011e: Revision of the Afrotropical species of the Philonthus circumcinctus species group (Coleoptera: Staphylinidae: Philonthina). Klapalekiana 47: 173-200.

HROMÁDKA L. 2011f: Revision of the genus Gabrius Stephens 1829 from Madagascar and adjoining Islands (Coleoptera: Staphylinidae: Philonthina). Linzer Biologische Beiträge 43: 1377-1397.

HROMÁDKA L. 2011g: Two new species of the genus Philonthus from the Afrotropical region (Coleoptera: Staphylinidae: Staphylininae: Philonthina). Linzer Biologische Beiträge 43: 1345-1350.

HROMÁDKA L. 2012a: A new species of Mentophilonthus from the Central African Republic (Coleoptera: Staphylinidae: Philonthina). Linzer Biologische Beiträge 44: 1155-1158.

HROMÁDKA L. 2012b: Revision of the Afrotropical species of the genus Hesperus Fauvel (Coleoptera: Staphylinidae: Philonthina). Linzer Biologische Beiträge 44: 551-589.

HROMÁDKA L. 2012c: Revision of Afrotropical species of the Philonthus interocularis species group (Coleoptera: Staphylinidae: Philonthina). Studies and Reports, Taxonomical Series 8: 137-178.

HROMÁDKA L. 2012d: Revision of the Afrotropical species of the Philonthus longicornis species group (Coleoptera: Staphylinidae: Staphylininae). Klapalekiana 48: 75-120.

HROMÁDKA L. 2012e: Revision of Afrotropical species of the Philonthus spinipes species group (Coleoptera: Staphylinidae: Staphylininae). Studies and Reports, Taxonomical Series 8: 175-196.

HROMÁDKA L. 2012f: Revision of the Philonthus discoideus - P. xanthoraphis group (Coleoptera: Staphylinidae: Philonthina). Acta Societatis Zoologicae Bohemicae 76: 53-83.

HROMÁDKA L. 2013a: A new species of Gabronthus Tottenham, 1955 from Republic of South Africa (Coleoptera: Staphylinidae: Philonthina). Studies and Reports, Taxonomical Series 9: 73-76.

HROMÁDKA L. 2013b: New species of the genus Philonthus from the Afrotropical region (Coleoptera: Staphylinidae: Staphylininae: Philonthina). Klapalekiana 49: 1-22.

HROMÁDKA L. 2013c: Revision of the Afrotropical species of the Philonthus maculipennis species group (Coleoptera: Staphylinidae: Philonthina). Acta Societatis Zoologicae Bohemicae 77: 187-202.

HROMÁDKA L. 2013d: Revision of Afrotropical species of the Philonthus politus species group (Coleoptera: Staphylinidae: Philonthina). Studies and Reports, Taxonomical Series 9: 379-456.

HROMÁDKA L. 2013e: Revision of the Afrotropical species of the Philonthus rudipennis species group (Coleoptera: Staphylinidae: Philonthina). Acta Societatis Zoologicae Bohemicae 77: 203-252.

HROMÁDKA L. 2013f: Two new species of the genus Philonthus from Afrotropical region with two nomenclatural changes in the genus (Coleoptera: Staphylinidae: Staphylininae: Philonthina). Linzer Biologische Beiträge 45: 713-788.

HROMÁDKA L. 2013g: Two new species of the genus Philonthus from Central African Republic (Coleoptera: Staphylinidae: Philonthina).
Linzer Biologische Beiträge 45: 703-708.

HROMÁDKA L. 2014a: A new species Afrorabigus scopus nov. sp. from Tanzania (Coleoptera: Staphylinidae: Philonthina). Linzer Biologische Beiträge 46: 683-686.

HROMÁDKA L. 2014b: A new species of the genus Gabronthus from the Afrotropical Region (Coleoptera: Staphylinidae: Philonthina). Studies and Reports, Taxonomical Series 10: 97-106.

HROMÁDKA L. 2014c: A review of the Afrotropical Gabrius coryndoni species group (Coleoptera: Staphylinidae: Staphylininae). Acta Entomologica Musei Nationalis Pragae 54: 555-562.

HROMÁDKA L. 2014d: Four new species of the genus Philonthus from Afrotropical region (Coleoptera: Staphylinidae: Philonthina). Linzer Biologische Beiträge 46: 687-694.

HROMÁDKA L. 2014e: Five new species of the genus Gabrius from Afrotropical region (Coleoptera: Staphylinidae: Staphylininae: Philothina). Linzer Biologische Beiträge 46: 695-702.

HROMÁDKA L. 2014f: New species of the genus Gabrius from the Afrotropical Region (Coleoptera: Staphylinidae: Philonthini) - I. Folia Heyrovskyana, Series A 22: 26-34.

HROMÁDKA L. 2014g: New species of the genus Gabrius from the Afrotropical Region (Coleoptera: Staphylinidae: Philonthini) - II. Folia Heyrovskyana, Series A 22: 35-53.

HROMÁDKA L. 2014h: Revision of the Afrotropical species of the Gabrius burgeoni species group (Coleoptera: Staphylinidae: Philonthina). Acta Societatis Zoologicae Bohemicae 78: 171-193.

HROMÁDKA L. 2014i: Revision of the Afrotropical species of the Gabrius chapmani species group (Coleoptera: Staphylinidae: Philonthina). Acta Societatis Zoologicae Bohemicae 78: 195-211.

HROMÁDKA L. 2014j: Six new species of the genus Philonthus from the Afrotropical region (Coleoptera: Staphylinidae: Philonthina). Studies and Reports, Taxonomical Series 10: 415-426.

HROMÁDKA L. 2015a: New species of the genus Philonthus from Tanzania (Coleoptera: Staphylinidae: Philonthini). Studies and Reports, Taxonomical Series 11: 59-64.

HROMÁDKA L. 2015b: New species of the Philonthina from the Afrotropical Region (Coleoptera: Staphylinidae: Philonthini). Studies and Reports, Taxonomical Series 11: 297-303.

HROMÁDKA L. 2015c: Three new Philonthini species from Ethiopia (Coleoptera: Staphylinidae: Philonthini). Folia Heyrovskyana, Serie A 23: 27-31.

HROMÁDKA L. 2016a: Four new Philonthina species (Coleoptera: Staphylinidae: Philonthina). Acta Societatis Zoologicae Bohemicae 80: 101-107.

HROMÁDKA L. 2016b: New Philonthina species from Tanzania (Coleoptera: Staphylinidae: Philonthini: Philonthina). Klapalekiana 52: 25-32.

HROMÁDKA L. 2016c: Six new Philonthini from the Afrotropical Region (Coleoptera: Staphylinidae: Philonthina). Studies and Reports, Taxonomical Series 12: 371-381.

HROMÁDKA L. 2016d: Two new species of the genus Gabrius from the Afrotropical Region (Coleoptera: Staphylinidae: Philonthini). Studies and Reports, Taxonomical Series 12: 93-98.

HROMÁDKAL. 2016e: Two new species of the genus Gabronthus from the Afrotropical Region (Coleoptera: Staphylinidae: Philonthina). Studies and Reports, Taxonomical Series 12: 383-386.

HROMÁDKA L. \& JANÁK J. 2011: Philonthus hapalemur sp. nov. and redescription of P. plasoni Bernhauer, 1902 from Madagascar (Coleoptera: Staphylinidae: Philonthina). Acta Entomologica Musei Nationalis Pragae 51: 137-143.

HROMÁDKA L. \& SCHILLHAMMER H. 2007: Mentophilonthus davidkrali sp. nov. from Nepal and India (Coleoptera: Staphylinidae: Staphylininae). Acta Societatis Zoologicae Bohemicae 71: 1-6.

ICZN 1999: International Code of Zoological Nomenclature. Fourth edition. The International Trust for Zoological Nomenclature, London, $306 \mathrm{pp}$.

JANÁK J. 2011: K osmdesátinám Lubomíra Hromádky. (Lubomír Hromádka 80 years old). Klapalekiana 47: 147-151 (in Czech and English).

JANÁK J. 2013: Two new species and additional records of the genus Philonthus from Madagascar (Coleoptera: Staphylinidae: Staphylininae). Klapalekiana 49: 23-38.

JANÁK J. 2017: In memoriam of Lubomír Hromádka. Studies and Reports, Taxonomical Series 13: 129-133. 
JANÁK J. \& LECOQ J.-C. 2005: Une nouvelle espèce et de nouvelles récoltes d'espèces du groupe Philonthus rufus Fauvel de Madagascar (Coleoptera, Staphylinidae, Philonthina). Nouvelle Revue d'Entomologie (Nouvelle Série) 21: 287-291.

KMENT P., KOLÍNOVÁ Z. \& HEISS E. 2015: Catalogue of type specimens of true bugs (Hemiptera: Heteroptera) deposited in the National Museum, Prague, Czech Republic. Pentatomomorpha: Aradidae. Acta Entomologica Musei Nationalis Pragae 55: 411-443.

MACHÁČKOVÁ L. \& FIKÁČEK M. 2014: Catalogue of the type specimens deposited in the Department of Entomology, National Museum, Prague, Czech Republic. Polyneoptera. Acta Entomologica Musei Nationalis Pragae 54: 399-450.

MACHÁČKOVÁ L., MIKÁTOVÁS̆. \&HÁJEK J. 2017: Catalogue oftype specimens of beetles (Coleoptera) deposited in the National Museum, Prague, Czech Republic. Staphylinidae: Euaesthetinae, Leptotyphlinae, Megalopsidiinae, Oxyporinae and Steninae. Acta Entomologica Musei Nationalis Pragae 57: 791-834.

MÄKLIN F. G. 1852. [New species and notes]. In: MANNERHEIM C. G. vON: Zweiter Nachtrag zur Kaefer-Fauna der Nord-Amerikanischen Laender des Russischen Reiches. Bulletin de la Société Impériale des Naturalistes de Moscou 25: 283-387.

MÄKLIN F. G. 1853. [New species and notes]. In: MANNERHEIM C. G. vON: Dritter Nachtrag zur Kaefer-Fauna der Nord-Amerikanischen Laender des Russischen Rieches. Bulletin de la Société Impériale des Naturalistes de Moscou 26: 95-273.

MALENOVSKÝ I., ZÁRUBA M. \& KMENT P. 2016: Catalogue of type specimens of Sternorrhyncha (Hemiptera) deposited in the National Museum, Prague, Czech Republic. Acta Entomologica Musei Nationalis Pragae 56: 423-446.

MANNERHEIM C. G. vON 1843: Beitrag zur Kaefer-Fauna der Aleutischen Inseln, der Insel Sitkha und Neu-Californiens. Bulletin de la Société Impériale des Naturalistes de Moscou 16: 175-314.

NEWTON A. F. 2015: New nomenclatural and taxonomic acts, and comments: Staphylinidae. Pp. 9-15. In: LÖBL I. \& LÖBL D. (eds.): Catalogue of Palaearctic Coleoptera. Volume 2. Revised and updated edition. Hydrophiloidea - Staphylinoidea. Brill, Leiden-Boston, xxv $+1702 \mathrm{pp}$.

ORTH R. E. \& MOORE I. 1980: A revision of the species of Cafius Curtis from the west coast of North America with notes of the east coast species (Coleoptera: Staphylinidae). Transactions of the San Diego Society of Natural History 19: 181-211.

PAULIAN R. 1952: Coléoptères. Pp. 90-103. In: DELAMARE A., DEBOUTTEVILlE C. \& PAULIAN R. (eds): Recherches sur la faune des nids et des terriers en Basse Côte d'Ivoire. Encyclopédie Biogéographique et Écologique 8: 1-116.

RAMBOUSEK F. G. 1920: Vědecké výsledky Československé armády v Rusku a na Sibiři. I. část. Nový rod ruských staphylinidů. (Résultat scientiphiques de l'armée tchécoslovaque en Russie et en Sibérie. Un genre nouveau des staphylinides de la Russie). Časopis Československé Společnosti Entomologické 17: 16-21 (in Czech and French).

RAMBOUSEK F. G. 1923:Vědecké výsledky Českoslov. armády v Rusku a na Sibiři. Résultats scientiphiques de l'armee tchécoslovaque en Russie et en Sibérie IV. Staphylinidae III. Časopis Československé Společnosti Entomologické 20: 54-55 (in French).

: Nové druhy jihoamerických drabčíků. (Staphylinides nouveaux de l'Amérique Méridionale (Col.)). Časopis Československé Společnosti Entomologické 21 [1924]: 69-76 (in French with Czech introduction).

SCHILLHAMMER H. 1998: Revision of the east Palaearctic and Oriental species of Philonthus Stephens - Part 1. The cyanipennis group (Coleoptera: Staphylinidae, Staphylininae). Koleopterologische Rundschau 68: $101-118$.

SCHILLHAMMER H. 1999: Nomenclatoral and distributional notes on the subfamily Staphylininae (Coleoptera: Staphylinidae). Entomological Problems 30: 61-62.

SCHILLHAMMER H. 2003: Revision of the East Palaearctic and Oriental species of Philonthus Stephens - Part 5. The rotundicollis and sanguinolentus species groups (Coleoptera: Staphylinidae, Staphylininae). Koleopterologische Rundschau 73: 85-136.

SCHILLHAMMER H. 2009: Notes on some West Palearctic Staphylinini, with description of a new species from Spain (Coleoptera: Staphyli- nidae: Staphylininae). Koleopterologische Rundschau 79: 97-116.

SCHILLHAMMER H. 2011: Old and new Staphylinini from the Palearctic and Oriental Regions (Coleoptera: Staphylinidae: Staphylininae). Koleopterologische Rundschau 81: 133-163.

SCHILLHAMMER H. 2019: New species and new faunistic data of West Palearctic Bisnius Stephens, 1829 (Coleoptera: Staphylinidae: Staphylininae). Koleopterologische Rundschau 89: 107-114.

SCHÜLKE M. 2012: A new name in the genus Philonthus Stephens 1829 (Coleoptera, Staphylinidae, Staphylininae). Linzer Biologische Beiträge 44: 1667.

SCHÜLKE M. \& SMETANA A. 2015: Family Staphylinidae Latreille, 1802. Pp. 304-1134. In: LÖBL I. \& LÖBL D. (eds.): Catalogue of Palaearctic Coleoptera. Volume 2. Revised and updated edition. Hydrophiloidea - Staphylinoidea. Brill, Leiden-Boston, xxv + 1702 pp.

SMETANA A. 1953a: Poznámky k rodu Gabrius Steph. (Col. Staphylinidae). Remarks on the genus Gabrius Steph. (Col. Staphylinidae) (5th contribution to the knowledge of the genus Gabrius Steph. of the Palaearctic Region). Acta Entomologica Musei Nationalis Pragae 28 [1952]: 165-180 (in English with Czech introduction).

SMETANA A. 1953b: Výsledky zoologické expedice Národního Musea v Praze do Turecka. Results of the zoological scientific expedition of the National Museum in Praha to Turkey. 12. Coleoptera III. Staphylinidae (genera Philonthus Curt., Gabrius Steph.). Acta Entomologica Musei Nationalis Pragae 28 [1952]: 117-124 (in English with Czech introduction)

SMETANA A. 1954a: Středoevropské druhy rodu Gabrius Steph. ze skupiny Gabrius splendidulus Grav. (Coleoptera, Staphylinidae). (4. př́spěvek k poznání rodu Gabrius Steph. Palaearktické oblasti). Die mitteleuropäischen Arten der Gattung Gabrius Steph. aus der Gruppe des Gabrius splendidulus Grav. (Coleoptera, Staphylinidae) (4. Beitrag zur Kenntnis der Gattung Gabrius Steph. der paläarktischen Region). Acta Entomologica Musei Nationalis Pragae 29: 113-119 (in German with Czech introduction).

SMETANAA. 1954b: Výsledky zoologické expedice Národního musea v Praze do Turecka. Results of the zoological scientific expedition of the National Museum in Praha to Turkey. 17. Coleoptera VI. Staphylinidae (genera Philonthus Curt., Gabrius Steph.). Acta Entomologica Musei Nationalis Pragae 29: 177-180 (in English).

SMETANAA. 1955: Beiträge zur Kenntnis der Gattung Philonthus Curt. II. (Coleoptera, Staphylinidae). Annales Historico-Naturales Musei Nationalis Hungarici (Series Nova) 6: 205-211.

SMETANA A. 1956: Systematické a faunistické poznámky ke zviŕreně drabčíků Československa II. (Zároveň 10. př́spěvek k poznání rodu Gabrius Steph. palearktické oblasti.). Systematische und faunistische Beiträge zur Kenntnis der Staphyliniden-Fauna der Tschechoslowakei II. (Zugleich 10. Beitrag zur Kenntnis der Gattung Gabrius Steph. der paläarktischen Region). Ročenka Československé Společnosti Entomologické 52 [1955]: 165-180 (in Czech and German).

SMETANA A. 1963: Beitrag zur Kenntnis der Staphyliniden-Fauna der Mongolei (Col., Staphylinidae). Acta Entomologica Musei Nationalis Pragae 35: 291-302.

SMETANA A. 1967: Zur Kenntnis der Gabrius-Arten Spaniens (Col., Staphylinidae). (80. Beitrag zur Kenntnis der Staphyliniden) Acta Faunistica Entomologica Musei Nationalis Pragae 12: 153-160.

SMETANA A. 1984: Review of the Japanese species of the genus Gabrius Stephens (Coleoptera, Staphylinidae). (121st contribution to the knowledge of Staphylinidae). Pan-Pacific Entomologist 60: 122-150.

SMETANAA. 1995: Rove beetles of the subtribe Philonthina of America north of Mexico (Coleoptera: Staphylinidae). Classification, phylogeny and taxonomic revision. Memoirs on Entomology, International 3: $1-946$.

SMETANA A. 1996. A new name in the genus Philonthus Stephens (Coleoptera: Staphylinidae, Staphylinini, Philonthina). Coleopterists Bulletin 50: 220.

TKOČ M., PECHAROVÁ M. \& JEŽEK J. 2014: Catalogue of the type specimens of Diptera deposited in the National Museum, Prague, Czech Republic. Moth flies (Psychodidae). Acta Entomologica Musei Nationalis Pragae 54: 789-837. 